\title{
Characterizing rainfall using microwave links
}

Thomas C. van Leth

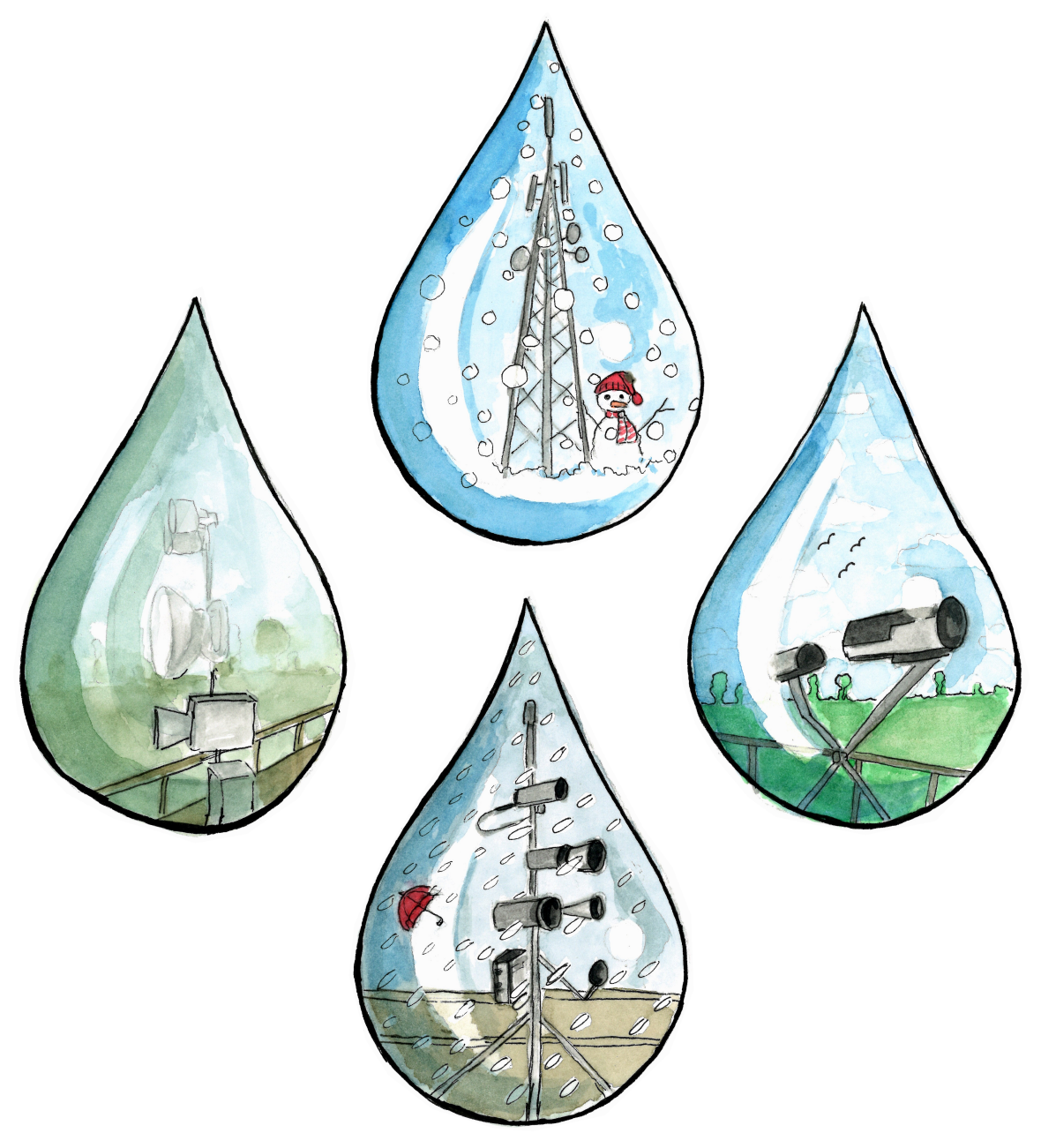




\section{Propositions}

1. To determine the response of a microwave link to rainfall, it is necessary to know the build of the antenna and transceiver.

(this thesis)

2. Dual-frequency/-polarization collocated microwave links can be used to fully characterize the raindrop size distribution for practical purposes, as long as the link metadata is known.

(this thesis)

3. Closed-source retrieval algorithms baked into measuring equipment are incompatible with open science.

4. Because the complexity and capabilities of hardware and operating systems are increasing, scripting languages can outperform low-level languages for modeling and data processing.

5. Programming is an essential skill for any independent and responsible $21^{\text {st }}$ century citizen and should be a compulsory part of secondary education.

6. An employer who does not commit to its employees for the long term cannot expect its employees to care about the company's future.

Propositions belonging to the thesis, entitled

Characterizing rainfall using microwave links

Thomas C. van Leth

Wageningen, 26 August 2019 



\section{Characterizing rainfall using microwave links}

Thomas Christiaan van Leth 


\section{Thesis committee}

\section{Promotor}

Prof. Dr R. Uijlenhoet

Professor of Hydrology and Quantitative Water Management

Wageningen University \& Research

\section{Co-promotors}

Dr H. Leijnse

Senior researcher, R\&D Observations and Data Technology

Royal Netherlands Meteorological Institute, De Bilt

DrA. Overeem

Researcher, R\&D Observations and Data Technology

Royal Netherlands Meteorological Institute, De Bilt

\section{Other members}

Prof. Dr A. Berne, École Polytechnique Fédérale de Lausanne, Switzerland

Prof. Dr A.A.M. Holtslag, Wageningen University \& Research

Prof. Dr H.W.J. Russchenberg, Delft University of Technology

Dr M. Cosset, Institut de Recherche pour le Développement, France

This research was conducted under the auspices of the Graduate School for SocioEconomic and Natural Sciences of the Environment (SENSE). 


\section{Characterizing rainfall using microwave links}

Thomas Christiaan van Leth

Thesis

submitted in fulfilment of the requirements for the degree of doctor

at Wageningen University

by the authority of the Rector Magnificus,

Prof. Dr A.P.]. Mol,

in the presence of the

Thesis Committee appointed by the Academic Board

to be defended in public

on Monday 26 August 2019

at 4 p.m. in the Aula. 
Thomas C. van Leth

Characterizing rainfall using microwave links, viii +112 pages .

PhD thesis, Wageningen University, Wageningen, the Netherlands (2019) With references, with summary in English

ISBN 978-94-6395-028-2

DOI 10.18174/495565 


\section{Abstract}

Microwave links are used to connect antennas on mobile telecommunication towers and can therefore be found almost everywhere there is mobile phone coverage. The link signals attenuate due to rainfall, and this fact can be applied to estimate the path-averaged rainfall rate along the path between the link antennas. The data obtained in this way can complement radar measurements mainly due to the fact that they measure rain closer to the ground and complements rain gauges because of their larger spatial sampling footprint. In many developing countries they have the potential to become the primary provider of rainfall measurements in combination with satellite measurements.

This thesis describes the setup and results of a field experiment in Wageningen, the Netherlands, aimed at assessing the error sources in microwave link measurements of rainfall and improving their measurement capabilities. The experiment consists of multiple collocated microwave links, one of which is a commercial off-the-shelf microwave link formerly part of an operational network. The microwave links are complemented with five disdrometers arranged along the link path. Using these instruments, the performance of a naive retrieval algorithm that does not take into account any sources of error is assessed. This retrieval results in significant overestimation. Next, I consider several different events where one or more non-rainfall attenuating phenomena are present: snowfall, dew, fog and clutter. I also analyze long-term variations in the received power level in periods without precipitation. These variations are found to be highly correlated to temperature.

The results of these analyses show remarkable differences between the responses of different microwave link instruments operating at the same frequency and polarization to some of these phenomena. In particular, the attenuation due to antenna wetting follows a completely different pattern between hydrophobic and hydrophilic antenna cover materials. The response for hydrophobic antenna covers includes a stochastic component and is therefore much harder to accurately compensate for in a retrieval algorithm. In general, the off-the-shelf microwave transceiver proves to be very stable and more reliable than the dedicated links, both in terms of stability of the signal in dry weather and predictability and severity of the effect of wet antennas.

The spatio-temporal variability of rainfall fields at the spatial scale of a single link is determined using the five disdrometers, located at different mutual distances. The decorrelation distance and intermittency distance are used as measures of the spatial variability pattern. Additionally, disdrometer data collected in Rotterdam and automatic gauge data from the KNMI nationwide network are used to extend the analysis over a wide range of scales. A single modified exponential function can characterize the rainfall decorrelation pattern over the entire range of scales to a reasonable degree. 
Using dual or triple collocated microwave links operating at different frequencies or with different polarizations, the average raindrop size distribution (DSD) along the link path can be obtained. In this thesis, an algorithm for retrieving the DSD is developed and its performance analyzed with the help of simulated attenuations based on the disdrometer measurements collected in Wageningen as well as an interpolated DSD field based on $\mathrm{X}$-band radar and disdrometer measurements from the Ardèche region, France. Based on the simulations it is found that dual collocated links in combination with a predetermined relationship between two gamma distribution parameters can perform just as well as triple collocated links in terms of accuracy, while also allowing for much faster computation. It is also found that dual-frequency retrievals are more sensitive to inaccuracies in the baseline power level than dual-polarization retrievals.

Finally, the DSD retrieval algorithm is applied to real microwave link measurements obtained in the Wageningen experiment. One event is chosen because of its relatively stable baseline power level. The microwave link derived DSD is close to the DSD derived from the disdrometer measurements for that same event, which proves the feasibility of the technique in practice. The far more stable baseline for the one off-the-shelf link (which could not be used for this analysis because if its single frequency and polarization) suggests that more large-scale DSD retrievals from operational networks are feasible. 


\section{Contents}

Abstract $\quad$ v

Contents vii

1 Introduction 1

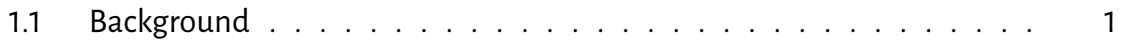

1.2 Overview of thesis . . . . . . . . . . . . . . . . . 6

2 A campaign to assess error sources in microwave link rainfall estimation 9

2.1 Introduction . . . . . . . . . . . . . . . . . . . . . . 11

2.2 Theoretical background . . . . . . . . . . . . . . . . . . 12

2.3 Experimental setup . . . . . . . . . . . . . . . . . . . . . . 16

2.3.1 Clobal overview . . . . . . . . . . . . . . . . . . 16

2.3.2 Microwave and near-infrared links . . . . . . . . . . . 16

2.3.3 Additional instruments . . . . . . . . . . . . . . . . . 17

2.4 Data processing . . . . . . . . . . . . . . . . . . . . . . . . 19

2.4 .1 Disdrometers . . . . . . . . . . . . . . . . . . . . . . . . . 19

2.4.1.1 Preprocessing . . . . . . . . . . . . . . . . 19

2.4.1.2 Derived data . . . . . . . . . . . . . . . . 19

2.4.1.3 Rainfall intensity - specific attenuation relationship 21

2.4 .2 Microwave links . . . . . . . . . . . . . . 22

2.5 Results and discussion . . . . . . . . . . . . . . . . . . 22

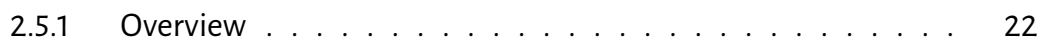

2.5 .2 Rainfall events . . . . . . . . . . . . . . . . . 23

2.5.3 Solid/mixed precipitation . . . . . . . . . . . . . 30

2.5.4 Temperature ....................... 32

2.5.5 Dew and fog . . . . . . . . . . . . . . . . . . . 34

2.5 .6 Wetantennas . . . . . . . . . . . . . . . . . . . . . . . . . . . 36

2.5 .7 Clutter . . . . . . . . . . . . . . . . . . 38

2.5.8 Compound phenomena ................ . . . 39

2.6 Conclusions . . . . . . . . . . . . . . . . . . . . . . . . 42

3 Rainfall spatio-temporal correlation and intermittency structure $\quad 45$

3.1 Introduction . . . . . . . . . . . . . . . . 46 
3.2 Data . . . . . . . . . . . . . . . . . . 46

3.2 .1 Datasets . . . . . . . . . . . . . . . . . 46

3.2.2 Quality control .................. . . . 47

3.3 Methodology . . . . . . . . . . . . . . . . . . . . . . . 49

3.4 Results . . . . . . . . . . . . . . . . . . . . 50

3.4.1 Spatial correlation ................ . . 50

3.4.2 Seasonality of spatial correlation . . . . . . . . . . . . . 54

3.4.3 Intermittency . . . . . . . . . . . . . . . . 55

3.5 Discussion . . . . . . . . . . . . . . . . . . . . . . . . 58

3.6 Conclusions . . . . . . . . . . . . . . . . . . . . . . 61

4 Estimating raindrop size distributions using microwave link measurements 63

4.1 Introduction . . . . . . . . . . . . . . . . . . . 64

4.2 Data . . . . . . . . . . . . . . . . . . . 65

4.2.1 Ardèche DSD reanalyses . . . . . . . . . . . . . . . . 65

4.2.2 Wageningen link experiment . . . . . . . . . . 66

4.3 Methods ....................... 67

4.3.1 Basic procedure . . . . . . . . . . . . . . 67

4.3.2 Three-parameter method ............. . . 70

4.3.3 Two-parameter method . . . . . . . . . . . . . 72

4.3 .4 Validation methods ................ 72

4.4 Validation using simulated DSD . . . . . . . . . . . . . . . . . 74

4.4 .1 Single retrieval . . . . . . . . . . . . . . . . . . . . 74

4.4 .2 Complete events . . . . . . . . . . . . . . . . 76

4.5 Retrieval from disdrometers . . . . . . . . . . . . . . . . . 77

4.5.1 Differences between two or three parameter retrievals . . . . 79

4.5.2 Dependence on link frequency . . . . . . . . . . . . . . . . 81

4.5 .3 Sensitivity to attenuation bias . . . . . . . . . . . . . . . 83

4.6 Experimental link retrieval . . . . . . . . . . . . . . . . . . . . . . . . . 83

4.7 Discussion . . . . . . . . . . . . . . . . . . . . . . . . . . 86

4.7 .1 Feasibility in practice . . . . . . . . . . . . . . . . . . 86

4.7 .2 Caveats . . . . . . . . . . . . . . . . 87

4.8 Conclusions and outlook . . . . . . . . . . . . . . . . . . . . . . 88

5 Synthesis 91

5.1 Summary and conclusions . . . . . . . . . . . . . . . . . . . . . 91

5.2 Recommendations for future research . . . . . . . . . . . . . . . . . 94

5.3 Application perspectives . . . . . . . . . . . . . . . . . 96

$\begin{array}{lr}\text { Bibliography } & 99\end{array}$

$\begin{array}{ll}\text { Statement of authorship contribution } & 106\end{array}$ 


\section{Chapter 1}

\section{Introduction}

\subsection{Background}

Accurate measurements and forecasts of precipitation are essential for many environmental applications. For instance, without it, hydrological models are useless and no flood or drought forecasts can be made (e.g. Braueret al., 2016). This in turn is essential for reducing loss of life and property due to hazardous weather and flooding. Especially in urban areas, the hydrological response to precipitation events can be extremely fast (as little as $30 \mathrm{~min}$ ) and also very local, with catchment sizes of the order of 100 ha. Therefore, high-resolution short-range forecasts are needed with spatial resolution less than $2 \mathrm{~km}$ and temporal resolution less than $3 \mathrm{~min}$ (Berne et al., 2004). These kinds of predictions, with forecast lead times up to a few hours at most, are usually referred to as nowcasting (e.g. Browning and Collier, 1989). For nowcasting to work, accurate and robust real-time high-resolution quantitative precipitation measurements are needed.

The most common instruments to measure rainfall are rain gauges (manual or automatic) (e.g. Strangeways, 2010) and weather radar (e.g. Fabry, 2015a). While individual rain gauges are less expensive than radar, they can only measure rainfall at specific locations; Indeed the total surface area covered by all rain gauges in the world is less than half a football field (Kidd et al., 2017). Because of the generally high spatial variability of rain, many rain gauges measuring simultaneously are needed to obtain a comprehensive picture of rainfall in a geographic area. Furthermore, rain gauges need regular maintenance. This means that obtaining countrywide coverage with rain gauges is an expensive proposition. In contrast, a single weather radar can cover a large area and provide information on the spatial distribution of rain at a high spatial and temporal resolution. Building and maintaining one radar is cheaper than maintaining an entire network of rain gauges for a similar spatial resolution. However, they still represent a significant one-time investment. Furthermore, the variables (reflectivity or e.g. specific differential phase) that they measure aloft is often difficult to relate quantitatively to the actual rain intensity at ground level without merging with rain gauge data. Therefore, a supporting network of rain gauges is still necessary. Radar transceivers also need to be placed away from tall 


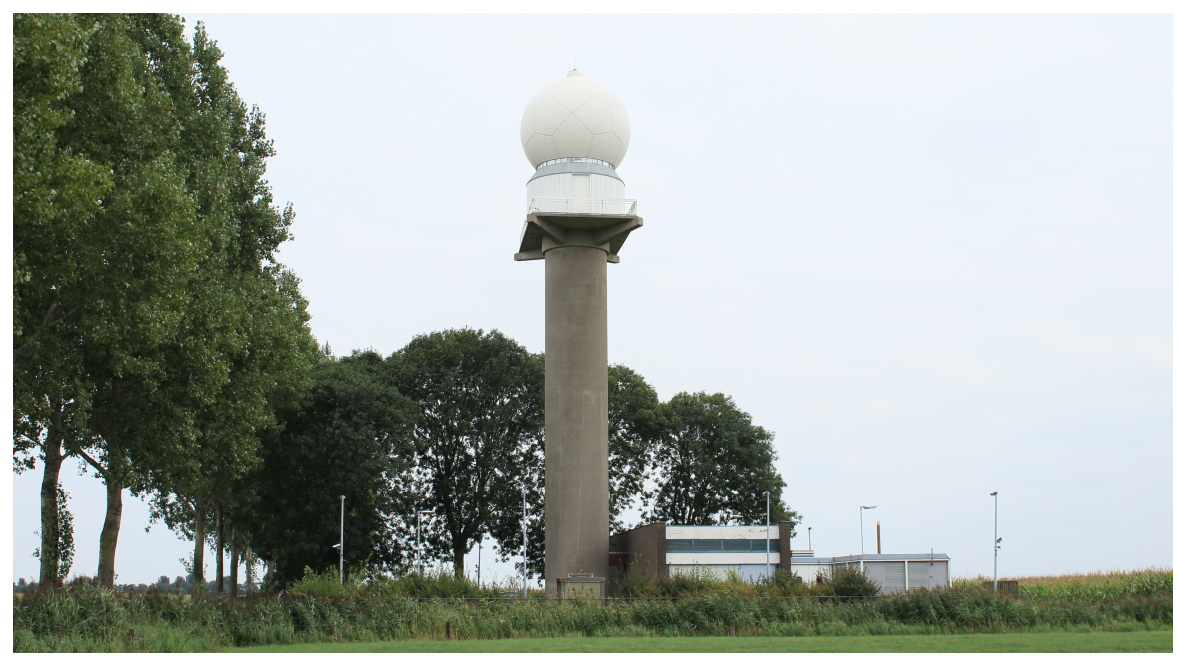

Figure 1.1: KNMI polarimetric C-band radar in Herwijnen. Photo courtesy of Aart Overeem.

buildings to avoid cluttering the radar image. Furthermore, they become less accurate at greater distance from the radar dome due to attenuation of the radar signal and variability in the vertical reflectivity profile. See Hazenberg et al. (2011) for an analysis of different radar error sources and correction mechanisms.

In addition to reflectivity, some radar systems can also measure polarimetric variables such as differential phase shift and differential reflectivity (which can be related to drop size and hydrometeor type) and Doppler frequency shift (which can be related to hydrometeor and hence wind velocity). In addition, special types of rain gauges called disdrometers (distribution of drops) are sometimes used that can measure individual raindrops and (for some types) their velocity: not just bulk rainfall. They can be based on either laser attenuation (e.g. Löffler-Mang and Joss, 2000), video (e.g. Schönhuberet al., 2008), pressure (e.g. Joss and Waldvogel, 1967), or simply filter paper (e.g. Marshall and Palmer, 1948; Wessels, 1972). Such instruments are more expensive than traditional gauges and not necessarily more reliable when it comes to rainfall measurement. They are mostly used in smaller areas and large-scale networks of disdrometers are scarce. However, some types, particularly laser disdrometers, are suitable for real-time measurement at high temporal resolution.

At the moment there remain large swaths of land surface area that are not covered by either radar or rain gauge networks. These are, for the most part, sparsely populated areas where providing accurate prediction services would not be cost effective. However, they also include some very densely populated areas in poorer regions of the world where governments cannot afford radar coverage, but accurate rainfall predictions are very much in demand. Currently, the only source of rainfall information for these areas comes from space-borne radars and radiometers such as the GPM (Clobal Precipitation 


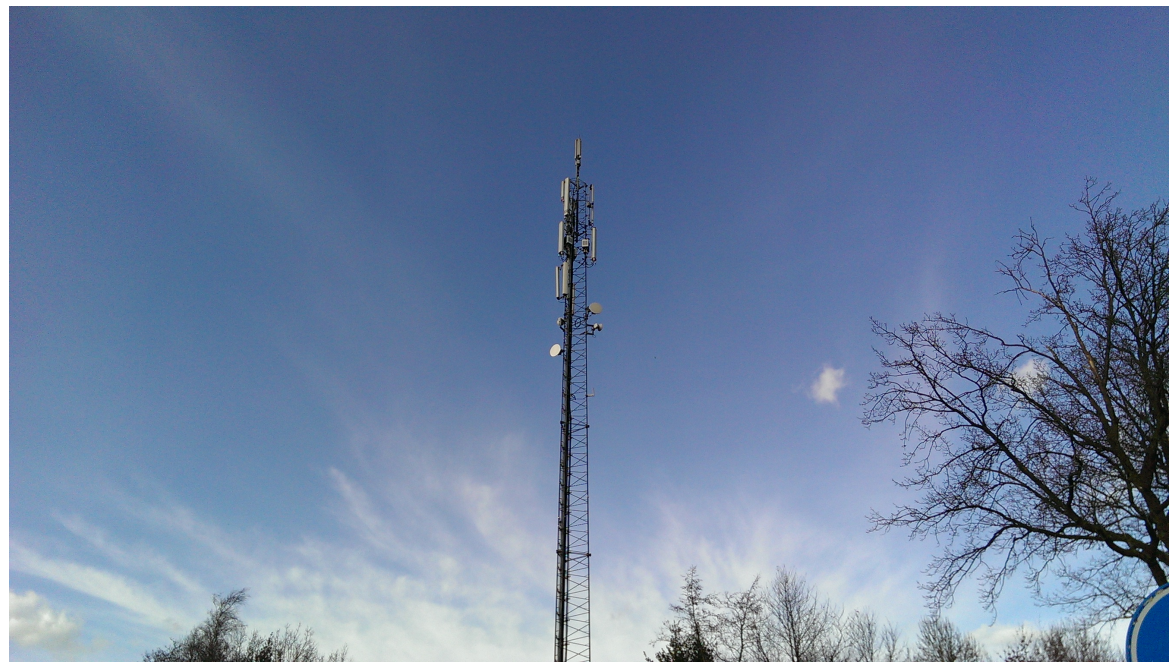

Figure 1.2: A mobile telecommunication tower. Note that the round antennas are the microwave transceivers used to communicate with other towers. It is these that can be used to measure rainfall.

Measurement) constellation in low-earth orbit (e.g. Skofronick-Jackson et al., 2018) and the optical and infrared passive sensors on board the MSC (Meteosat Second Ceneration) satellites in geostationary orbit among others (McCabe et al., 2017). This means only coarse spatial resolution observations and, in the case of LEO systems, high temporal intermittency. These kinds of instruments are useful for their large spatial coverage and can provide a reasonable accuracy (Rios Gaona et al., 2016) that is not far off from radar. However, their spatial resolution is inferior to ground-based radar and too coarse for dense urban areas. Furthermore, just like radar, their measurements need to be adjusted with rain gauge measurements to provide quantitative information suitable for hydrological purposes.

Microwave links were first used as rainfall measuring devices by Atlas and Ulbrich (1977), but received increased attention in the last 13 years (Uijlenhoet et al., 2018). They can fill some of the gaps in the current array of instruments, especially in geographical regions that are now poorly served, such as mountainous areas, cities and developing countries. A single microwave link consists of two devices: a transmitter and a receiver. They are placed typically a few hundreds of meters to a few kilometers apart. Variations in signal loss (attenuation) between the transmitter and receiver are dependent on the atmospheric conditions along the intermediate path. As the presence of raindrops in the air causes attenuation of the signal and the magnitude of the attenuation is proportional to the number and sizes of raindrops along the path, a well-calibrated link can be used to measure rainfall intensity. 


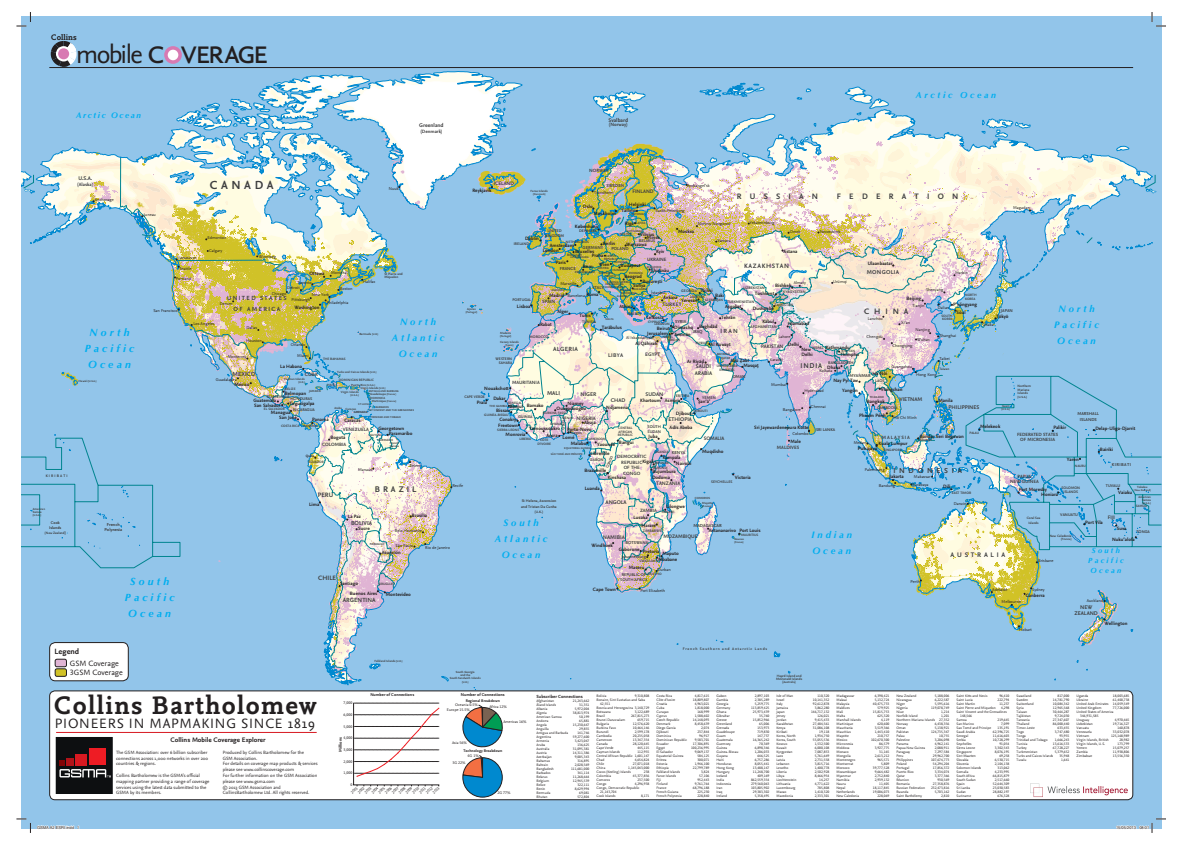

Figure 1.3: World map with 2013 cellular telephone coverage from the CSM Association (CSMA). (c) CSMA and Collins Bartholomew.

The microwave signal loss in rain is related to the complex microwave scattering characteristics of the individual rain drops along the path. Because the wavelength of the signal is in the order of millimeters and the diameters of the rain drops is of the same order, the attenuation due to scattering is strongly and nonlinearly dependent on the ratio of diameter and wavelength (Mie, 1908). Furthermore, raindrops are not perfect spheres, but rather oblate spheroids. Their oblateness is caused by the interaction with the ambient air as they fall and is directly related to their overall mass. For this reason the scatteringinduced attenuation of a polarized microwave signal is dependent on the polarization. Hence, the difference between attenuation of horizontally and vertically polarized signals is also dependent on the diameters of the raindrops. Therefore, the total signal loss over the link path and the difference between these losses at horizontal and vertical polarization is a function of the concentration and size distribution of drops aggregated over the path.

The big advantage of the use of microwave links for measuring rainfall, aside from their proximity to the ground, is their ubiquity. Although dedicated rainfall measuring microwave links are uncommon at the moment, a large global network of them already exists. In Fig. 1.3 the global coverage of mobile phone networks is shown; These networks rely on antenna masts, such as the one shown in Fig. 1.2. These masts often communicate with each other using microwave links and the signal strength of each of these microwave 


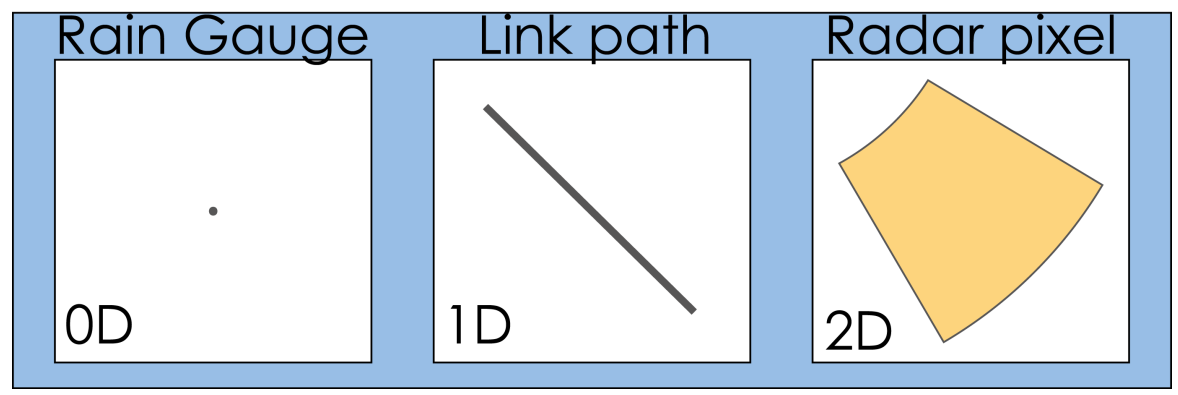

Figure 1.4: Artist's impression of the sampling footprints of rain gauges, microwave links and radars.

links is regularly recorded to assess the stability of the network. Although these were never designed as rainfall measurement devices, they can be used as such; This concept is often referred to as "opportunistic sensing" (McCabe et al., 2017). The idea of using these networks as rainfall measuring devices was first suggested by Upton et al. (2005) and then demonstrated by Messer et al. (2006) and Leijnse et al. (2007a). Microwave link coverage has been rapidly spreading worldwide and already extends to those densely populated areas in poorer regions of the world that were not covered by traditional rainfall measuring methods as shown in Fig. 1.3. There is an opportunity in those areas for microwave links to take on the role of primary rainfall data providers (in combination with existing satellite instruments) without the need for additional infrastructure.

The properties of microwave links give them a unique type of spatial sampling footprint as compared to gauges and radars. Whereas the footprint of a gauge is essentially zerodimensional because of its small size compared to the typical size of rainfall fields and radar provides a two-dimensional grid of projected three-dimensional volumes, the narrow beam of a link means its footprint is essentially one-dimensional (shown in Fig. 1.4). There are also differences in the location of the sampling volumes of these devices. Whereas rain gauges and disdrometers measure close to the ground, radar sampling volumes can actually be up to several kilometers altitude and increasing in altitude with distance from the radar. This means that these measurements are not necessarily representative for the actual rainfall depth at ground level due to e.g. the heterogeneous vertical profile of reflectivity (VPR) and rainfall evaporating before it reaches the ground i.e. virga (see Hazenberg et al. (2013) for a VPR mitigation strategy). Microwave links are again an intermediate between the two, usually measuring at several meters to tens of meters above ground level. Thus they are much more representative of the actual rainfall at ground level and also more comparable to rain gauges than radar is. Furthermore, the size of their sampling footprint is especially appropriate for the spatial scales involved in urban hydrology. The unusual sampling footprint of microwave links make it also interesting and important to look at the spatio-temporal variability at this small scale and determine how much the spatial representativeness can be improved over the use of rain gauges. 


\subsection{Overview of thesis}

The overall aim of this thesis is to assess and further develop the potential of microwave links for rainfall measurement on a fundamental level. There are four components to this: 1) assessment and mitigation strategies for errors in such measurements; 2) exploring the extraction of additional information about precipitation from the signal; 3 ) assessing the rainfall statistical characteristics at the typical link scale; and 4) increasing the overall accuracy of such measurements at the level of a single link.

In order to further this aim, a field experiment was started in the summer of 2014 and continued until early January 2016. It primarily concerned a number of microwave links deployed on a single path across the town of Wageningen together with several ancillary instruments. Most of the results discussed in this thesis are drawn from data obtained in this field experiment. The field experiment is described in Chapter 2: A campaign to assess error sources in microwave link rainfall estimation. The combination of several different microwave links along the same path including one former operational telecommunication link and several other auxiliary instruments may be the most comprehensive microwave link study to date.

Chapter 2 also describes the results of this experiment and gives an overview of factors influencing the reliability and accuracy of rainfall measurements. It assesses the severity of these influences based on the measured data and illustrates this with several case studies plucked from the field campaign. The use of time-lapse camera footage provided a unique qualitative understanding of surprising phenomena which would have otherwise remained hidden.

The presence of five disdrometers at different distances along the link path provides a unique opportunity to measure spatio-temporal characteristics of variability of the rainfall field at the sub-kilometer scale. Chapter 3: Rainfall spatio-temporal correlation and intermittency structure presents an analysis of the spatial structure at a typical link scale measured in Wageningen. We then extend these analyses to larger spatial scales using a disdrometer network in Rotterdam and the KNMI nationwide automatic gauge network and provide a multi-scale characterization of rainfall for the Dutch climate. We then compare with earlier studies carried out over a range of scales.

In Chapter 4: Parameterizing raindrop size distributions using microwave links a novel method to retrieve drop size distributions using collocated microwave link measurements is presented. It assesses the potential accuracy of, and presents the optimal configuration for such a retrieval to give the best results. It does this on the basis of simulated microwave link variables derived from the disdrometer measurements present in the field experiment as well as simulated DSD fields for two events based on polarimetric radar measurements in the Ardèche, France.

The combination of links used in the Wageningen experiment is uniquely suited for applying the methods developed in Chapter 4. This is demonstrated by the use of the DSD 
retrieval method on actual microwave link data. The results of this retrieval are presented in Chapter 4 as well.

In Chapter 5: Synthesis I use the combined results of the previous chapters to come to the main conclusions and further thoughts. I also suggest avenues for future research to capitalize and build on the work of this thesis. Finally, I will provide perspectives and recommendations towards practical application of the proposed techniques and microwave link rainfall estimation in general. 


\title{
Chapter 2
}

\section{A campaign to assess error sources in microwave link rainfall estimation}

\begin{abstract}
We present a measurement campaign to address several error sources associated with rainfall estimates from microwave links in cellular communication networks. The core of the experiment is provided by three co-located microwave links installed between two major buildings on opposite sides of the small town of Wageningen, approximately $2 \mathrm{~km}$ apart: a $38 \mathrm{CHz}$ formerly commercial microwave link, as well as 26 and $38 \mathrm{CHz}$ (dualpolarization) research microwave links. Transmitting and receiving antennas have been attached to masts installed on the roofs of the two buildings, about $30 \mathrm{~m}$ above the ground. This setup was complemented with an infrared large-aperture scintillometer, installed over the same path, as well as five laser disdrometers positioned at several locations along the path and an automated rain gauge. Temporal sampling of the received signals was performed at a rate of $20 \mathrm{~Hz}$. The setup was monitored by time-lapse cameras to assess the state of the antennas as well as the atmosphere. The experiment was active between August 2014 and December 2015. Data from an existing automated weather station situated just outside Wageningen was further used to compare and to interpret the findings. In addition to presenting the experiment, we also conduct a preliminary global analysis and show several cases highlighting the different phenomena affecting received signal levels: rainfall, solid precipitation, temperature, fog, antenna wetting due to rain or dew, and clutter. We also briefly explore cases where several phenomena play a role. A rainfall intensity $(R)$-specific attenuation $(k)$ relationship was derived from the disdrometer data. We find that a basic rainfall retrieval algorithm without corrections already provides a reasonable correlation to rainfall as measured by the disdrometers. However, there are strong systematic overestimations (factors of 1.2-2.1) which cannot be attributed to the $R-k$ relationship. We observe attenuations in the order of $3 \mathrm{~dB}$ due to antenna wetting under fog or dew conditions. We also observe fluctuations of a similar magnitude related to changes in temperature. The response of different makes of microwave antennas to many of these phenomena is significantly different even under the exact same operating conditions and configurations.
\end{abstract}


Chapter 2. A campaign to assess error sources in microwave link rainfall estimation

This chapter is based on: Van Leth, T.C., Overeem, A., Leijnse, H. and Uijlenhoet, R., A measurement campagin to assess sources of error in microwave link rainfall estimation, Atmos. Meas. Tech, 11, 4645-4669, doi:10.5194/amt11-4645-2018, 2018. 


\subsection{Introduction}

Accurate and real-time precipitation measurements are important for flood prediction, especially in urban areas. Traditional measurement techniques such as rain gauges have an insufficient temporal and spatial network density to allow for accurate measurements in an urban setting (Schilling, 1991; Berne et al., 2004). Furthermore, their spatial representativeness is limited because of their small sampling areas, making them essentially zero-dimensional point measurements. Weather radars, in contrast, have a much larger sampling area and provide full coverage making their observations more representative of the spatial precipitation distribution, but their space-time resolution is often limited, in particular for urban applications. Furthermore, radar observations take place higher up in the atmosphere the further away they are from the radar antenna (typical observation heights can be more than $1000 \mathrm{~m}$ ). Therefore, their measurements may not be representative of the situation near ground level. Finally, their high cost may be prohibitive for use by developing countries or local authorities.

Microwave link measurements may be a promising addition to the existing range of rain measurement techniques. The use of such instruments for measuring rainfall was first suggested by Atlas and Ulbrich (1977). With respect to spatial representativeness and resolution, microwave links can fill some of the gaps between rain gauges and weather radar. The area sampled is along the path of the link: typically about a few kilometres long and a few metres to dozens of metres wide at the widest point. This makes the sampling footprint approximately one-dimensional. This is more spatially representative than a rain gauge, but less so than radar. However, microwave links have two major advantages over radar: they measure much closer to the ground than radar (typically a few tens of metres), and the relation between the measured variable (specific attenuation in the case of links and radar reflectivity in the case of traditional radar) and rainfall intensity is much better defined and closer to linear for microwave links. Despite these advantages, microwave links had not been deployed at a large scale for the purpose of rainfall monitoring, for the cost of setting up such a network would still have been quite severe. The real potential of microwave link measurements for rainfall measurement came with the realization that the microwave links used in cellular communications networks could be repurposed as rainfall measurement devices, which was demonstrated by Messer et al. (2006) and Leijnse et al. (2007a). Doing so eliminates most of the cost of this technique as existing infrastructure can be used. This is especially valuable in developing countries, which typically have few rain gauges let alone weather radar, yet often do have an extensive mobile phone network (Doumounia et al., 2014). In the recent past there have been a number of studies about the application of commercial microwave link networks for rainfall measurements. These studies have demonstrated the feasibility of this method in southern Cermany (Chwala et al., 2012), the Netherlands (Overeem et al., 2011, 2013), Israel (Zinevich et al., 2008, 2009) and also in Burkina Faso (Doumounia et al., 2014) and Brazil (Rios Caona et al., 2018).

Although the rainfall maps produced by this method show surprisingly good correspondence with the gauge-adjusted radar product (Overeem et al., 2013), there are still inaccu- 
racies remaining in the final products (Leijnse et al., 2008, 2010; Zinevich et al., 2010). Error sources can generally be divided into errors due to the mapping of the rainfall estimates from the microwave links, and errors in the individual measurements and the rainfall retrieval algorithm. It is in this last category where the largest remaining sources of error reside and not in the mapping (Rios Caona et al., 2015). Therefore, further research is needed regarding the physical aspects of the attenuation measurements themselves. Several possible sources of error affecting the quality of rainfall retrievals from single microwave links have been identified previously: the wet antenna effect and related dew formation on antennas (Minda and Nakamura, 2005; Leijnse et al., 2008; Schleiss et al., 2013), humidity (Holtet al., 2003) and temperature (Minda and Nakamura, 2005), solid precipitation, and spatial variability of rainfall (Berne and Uijlenhoet, 2007). Opportunities for simultaneous measurement of environmental variables other than rainfall have also been identified, such as evaporation (Leijnse et al., 2007b), fog (Liebe et al., 1989; David et al., 2013), humidity (Chwala et al., 2013), and hydrometeor type (Cherkasskyet al., 2014).

In this chapter we describe a dedicated microwave link experiment that has been set up in the college town of Wageningen and present an analysis of the results. The field experiment was designed to provide validation data for microwave link rainfall retrieval at the scale of a single link, and to be able to compare different types of links simultaneously measuring along the same path. The goal of the analysis is to give a comprehensive overview of the phenomena encountered by a typical microwave link and to evaluate their relevance to a rainfall intensity retrieval. In order to do so we employ a relatively straightforward retrieval algorithm with a minimum number of corrections and make use of a number of auxiliary measurement devices to gain insight into the retrieved signal. In Sect. 2.2, a brief overview of the theoretical background pertaining to the operating principles of microwave link rainfall measurements is given. Section 2.3 covers a description of the experimental setup and the employed instruments. In Sect. 2.4, the data processing methods applied in this experiment are detailed. In Sect. 2.5, the obtained experimental data are presented and an inventory of encountered phenomena is given. Finally, in Sect. 2.6 conclusions are drawn.

\subsection{Theoretical background}

Both the attenuation of a microwave signal by rain drops during a rain event and the corresponding rainfall intensity can be related to the rain drop size distribution. The rainfall intensity (in $\mathrm{mm} \mathrm{h}^{-1}$ ) can be calculated as follows, assuming the density of water to be constant:

$$
R(t)=C_{\mathrm{R}} \int_{0}^{\infty} V(D) v(D) N(D, t) \mathrm{d} D .
$$

Here $V(D)$ is the volume of a raindrop in $\mathrm{mm}^{3}, D$ is the raindrop diameter in $\mathrm{mm}, v(D)$ is the fall velocity (in $\mathrm{m} \mathrm{s}^{-1}$ ) of a particle with diameter $D$ and $N(D, t)$ is the density of 
particles with diameter $D$ per $\mathrm{m}^{3}$ or drop size distribution (DSD) as a function of time $t$ and $C_{\mathrm{R}}=3.6 \times 10^{-3}$ is a unit conversion factor. When dividing the particle diameter into discrete classes (as is measured by a disdrometer) this can be approximated as follows:

$$
\begin{aligned}
& R(t)=C_{\mathrm{R}} \frac{1}{6} \pi \int_{0}^{\infty} D^{3} v(D) N(D, t) \mathrm{d} D \\
& \approx C_{\mathrm{R}} \frac{1}{6} \pi \sum_{i=1}^{n} D_{i}^{3} v\left(D_{i}\right) N\left(D_{i}, t\right) \Delta D_{i} .
\end{aligned}
$$

Here, $D_{i}$ is the mean diameter of the $i$-th drop size class, $N\left(D_{i}, t\right)$ is the discrete drop size distribution. $\Delta D_{i}$ is the width of the $i$-th diameter class, and $n$ is the number of drop size classes. A similar function defines the specific (logarithmic) attenuation (in $\mathrm{dB} \mathrm{km^{-1 }}$ ), where we assume that the particle density is low enough such that multiple scattering can be neglected:

$$
\begin{aligned}
& k(t)=C_{k} \int_{0}^{\infty} \sigma_{\text {ext }}(D) N(D, t) \mathrm{d} D \\
& \approx C_{k} \sum_{i=1}^{n} \sigma_{\text {ext }}\left(D_{i}\right) N\left(D_{i}, t\right) \Delta D_{i} .
\end{aligned}
$$

Here, $\sigma_{\text {ext }}(D)$ (in $\mathrm{mm}^{2}$ ) is the extinction cross-section of a hydrometeor with a diameter $D$ and $C_{k}=100 \cdot \ln (10)^{-1}$ is a unit conversion factor. $\sigma_{\text {ext }}(D)$ is also dependent on the frequency and polarization of the incident radiation. It can be derived from the forward scattering amplitude matrix $\mathbf{S}(D)$, which relates the incoming electromagnetic wave with the outgoing (forward scattered) wave,

$$
\begin{gathered}
\left(\begin{array}{c}
E_{\mathrm{h}} \\
E_{\mathrm{v}}
\end{array}\right)=\mathbf{S}(D)\left(\begin{array}{c}
E_{\mathrm{h} 0} \\
E_{\mathrm{v} 0}
\end{array}\right)=\left(\begin{array}{cc}
S_{\mathrm{hh}}(D) & S_{\mathrm{hv}}(D) \\
S_{\mathrm{vh}}(D) & S_{\mathrm{vv}}(D)
\end{array}\right)\left(\begin{array}{c}
E_{\mathrm{h} 0} \\
E_{\mathrm{v} 0}
\end{array}\right), \\
\left(\begin{array}{c}
\sigma_{\text {ext }, \mathrm{h}}(D) \\
\sigma_{\text {ext }, \mathrm{v}}(D)
\end{array}\right)=\frac{\lambda^{2}}{\pi} \Im\left[\left(\begin{array}{c}
S_{\mathrm{hh}}(D) \\
S_{\mathrm{vv}}(D)
\end{array}\right)\right],
\end{gathered}
$$

where $\lambda$ is the wavelength of the radiation in $\mathrm{mm}, S_{i j}$ is the element of the scattering amplitude matrix for the component of radiation with incoming polarization $i$ and outgoing polarization $j$, where $v$ and $h$ represent the vertically polarized and horizontally polarized components, respectively and $\Im$ is an operator indicating the imaginary part of it's argument (e.g. Van der Hulst, 1957). The forward scattering amplitude matrix for spheres of arbitrary size and dielectric properties can be calculated with Mie scattering theory 
(Mie, 1908). In order to be able to calculate the scattering properties for non-spherical drop shapes, we make use of the T-matrix approach (Waterman, 1965; Mishchenko et al., 1996).

The relationships between the raindrop diameter on the one hand, and the raindrop fall speed and its extinction cross-section on the other, closely resemble power laws (e.g. Atlas and Ulbrich, 1977). This means that both the specific attenuation and the rainfall intensity are approximately statistical moments of the DSD, which can themselves be empirically related by a power-law,

$$
R=a k^{b},
$$

where $a$ and $b$ are fitted parameters (Atlas and Ulbrich, 1977), which are both dependent on the average DSD as well as the temporal and spatial distribution of the DSD within the measured volume. Due to the dependency on these DSD characteristics, power-law parameters derived from a particular set of observations would strictly speaking only be valid for links that have the exact temporal and spatial distribution of drop sizes and concentrations within their path. This would mean that, even under the assumption of a uniform and unchanging DSD for a given climate, rainfall variability and intermittency within the link path volume as a rain event evolves or passes over would lead to inaccurate estimation of $R$ with this method. However, at the carrier wave frequencies typically employed in cellular communications links, the integrands in Eq. (1) and Eq. (2) are of a similar magnitude. As a result, the $R-k$ relationship is almost independent of the DSD and the exponent $b$ is close to 1 (Olsen et al., 1978; Leijnse et al., 2007c). Therefore, an $R-$ $k$ relationship derived for this range of frequencies could be valid for a broad range of events. Furthermore, because of the near-linearity of the relationship, parameters derived from either point measurements of the DSD or path-averages of the DSD can be used to derive path-average rainfall intensities from path-integrated attenuation in heterogeneous rain fields:

$$
\langle R\rangle=\frac{1}{L} \int_{0}^{L} a k(s)^{b} \mathrm{~d} s \approx \frac{1}{L} a\left[\int_{0}^{L} k(s) \mathrm{d} s\right]^{b}=\frac{1}{L} a A^{b} .
$$

Here $L$ is the length of the link path, $k(s)$ is the specific attenuation at position $s$ and $A$ is the path-integrated attenuation.

The microwave link precipitation detection method is principally intended for liquid precipitation. Snow and hail have different electromagnetic characteristics (i.e. ice has a different refractive index than water, and the shapes of the particles are different). Therefore, different attenuation-precipitation relations hold. Non-melting snow flakes cause very little attenuation in the frequency range under study (e.g. Battan, 1973) and thus we do not expect to be able to detect them. Wet snow hydrometeors, on the other hand, 

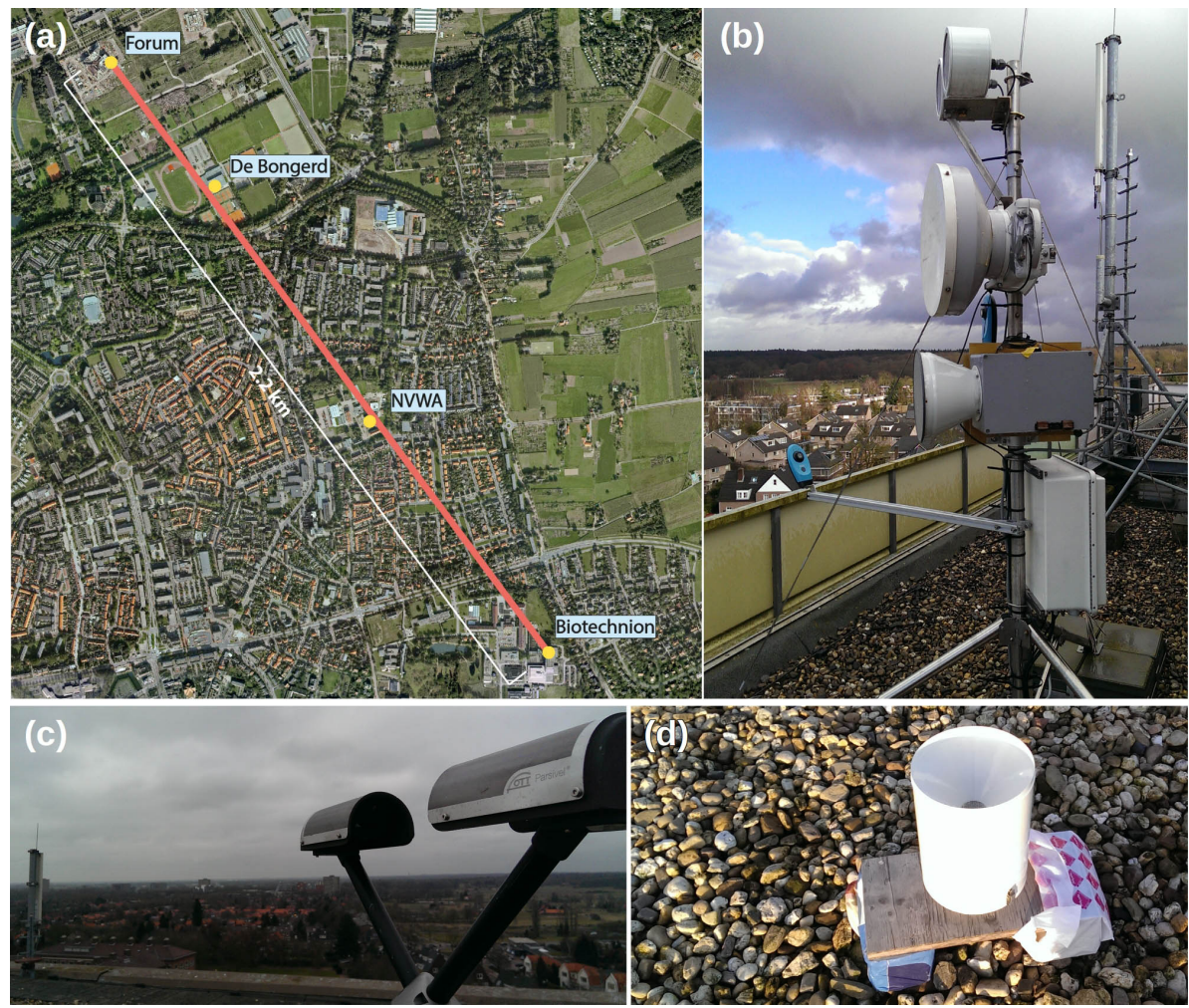

Figure 2.1: (a) A map of Wageningen showing the path of the links in red. The receiving antennas are at the end labelled "Forum"; the transmitting antennas are positioned at the end labelled "Biotechnion". The positions of the disdrometers are indicated with yellow dots. Each dotted position houses one disdrometer, except at the "Forum" position, where two disdrometers and an additional tipping bucket rain gauge are placed. (b) The transmitting antenna mast placed on the roof of the "Biotechnion" building. From top to bottom: Scintec BLS900, Nokia Flexihopper (38 GHz) and RAL $26 \mathrm{CHz}$. The RAL $38 \mathrm{CHz}$ is placed behind the RAL $26 \mathrm{CHz}$ in the photo's perspective and thus not visible. (c) A Parsivel disdrometer (on the "Biotechnion" site). (d) Précis Méchanique tipping bucket rain gauge at the "Forum" site.

which consists of a mixture of solid and liquid water and air, generally cause more microwave attenuation than a raindrop containing the same amount of water. As we are dealing with more complex shapes and multiple phases of water and air and thus an inhomogeneous index of refraction, accurate estimates of wet snow attenuation and inversely, the estimation of snowfall magnitude through microwave attenuation, poses a real challenge (e.g. Paulson and Al-Mreri, 2011). Nevertheless, we could still detect the presence of wet snow and melting ice pellets. 


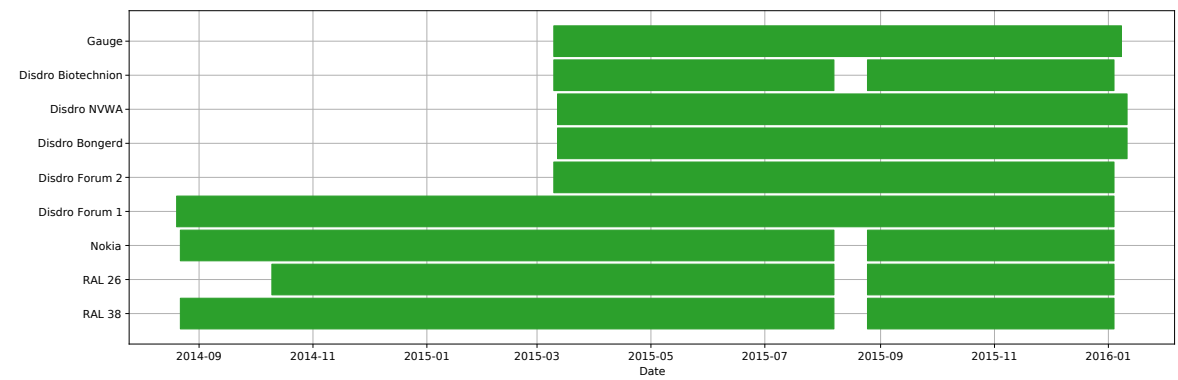

Figure 2.2: Operational period per instrument in the experimental setup.

\subsection{Experimental setup}

\subsubsection{Global overview}

The backbone of the experimental setup consists of three microwave links placed along the same path between two university buildings on opposite sides of the college town of Wageningen. As such, the majority of the $2.2 \mathrm{~km}$ long link path covers urban terrain (Fig. 2.1a). All transmitting antennas are placed on a $2 \mathrm{~m}$ high mast, approximately $1.5 \mathrm{~m}$ from the base of the mast (Fig. 2.1b). The mast is placed on top of a seven storey building. The building is situated atop a slightly elevated area on the south end of Wageningen $\left(51.969^{\circ} \mathrm{N}, 5.683^{\circ} \mathrm{E}\right)$. The receiving antennas are placed on an identical mast on the roof of an eight storey building at the northern end of Wageningen $\left(51.985^{\circ} \mathrm{N}, 5.664^{\circ} \mathrm{E}\right)$. The height above ground level is $27 \mathrm{~m}$ on the transmitting end and $40 \mathrm{~m}$ on the receiving end. The total height above sea level is $62 \mathrm{~m}$ at the transmitting end and $51 \mathrm{~m}$ at the receiving end. The terrain in between the endpoints of the path consists mostly of terraced housing, a sports field and other buildings of three stories or less. The maximum width of the first Fresnel zone (halfway along the path) at the featured frequencies is less than $5 \mathrm{~m}$. Thus, considering the height of the antenna locations compared to the intermediate terrain, there are no permanent obstructions affecting the beam significantly.

The experiment was operational from 22 August 2014 up to and including 8 January 2016. Not all instruments had been operational during this entire period though, as is indicated in Fig. 2.2. Also, from 7 to 25 August 2015 all transmitters were nonoperational due to a local power outage.

\subsubsection{Microwave and near-infrared links}

Of the three links one is a Nokia Flexihopper (Nokia), formerly part of a commercial mobile phone network operated by T-Mobile Netherlands. Such links are still used in mobile phone networks around the world and this microwave link could thus be regarded as a typical example of the link systems that would be used in an operational setting. The Nokia is a bidirectional link, but only one receiver was logged in this experiment. 
It is set to transmit and receive at a frequency of $38.17625 \mathrm{CHz}$ in one direction (which was recorded) and $39.43625 \mathrm{CHz}$ in the other direction. The bandwidth of the signals is $0.9 \mathrm{MHz}$. The device transmits and receives only horizontally polarized radiation.

The other two links are custom-built by Rutherford Appleton Laboratories (UK) (RAL). The first operates at $26.00000 \mathrm{CHz}$ and transmits and receives only horizontally polarized radiation. It contains both a linear and a logarithmic detector. The second RAL link operates at $38.00000 \mathrm{GHz}$. The bandwidth of the receivers is $4 \mathrm{KHz}$, while the transmitted signal bandwidth is extremely narrow $(\ll 1 \mathrm{KHz})$. Their oscillators are locked to GPS and thus extremely stable. The receiver contains four detectors, two of which measure horizontally polarized radiation (linear and logarithmic) and the other measures vertically polarized radiation (idem). The phase difference between the horizontally and vertically polarized signals is measured by separate detectors as well. In this chapter we will only deal with data from the logarithmic detectors. Note that the second RAL link measured at roughly the same frequency as the Nokia link. The frequencies are chosen to be far enough apart so as not to cause interference, but are close enough that the scattering characteristics of the radiation with respect to raindrops are almost identical. Additional characteristics of the link antennas are given in Table 1.

A Scintec BLS900 near-infrared boundary layer scintillometer is also placed together with the microwave links on the same path. It operates at a frequency of $340 \mathrm{THz}(880 \mathrm{~nm})$. This provides information about, for example, fog and other visibility-affecting phenomena. Similarly to the microwave links (despite operating in a different scattering regime), it could potentially also be used to estimate rain intensity (Uijlenhoet et al., 2011).

All link receivers are sampled with a Campbell Scientific CR1000 data logger and stored on a remote server on a daily basis. The sampling frequency is $20 \mathrm{~Hz}$. Auxiliary data (e.g. operating temperature) is sampled at a frequency of $2 \mathrm{~min}^{-1}$. The Nokia system consists of separate outdoor and indoor units, the latter containing the digital signal processing circuits and power supply. Note that we have not actively used the indoor unit of the Nokia link system aside from the power supply; Instead, the analogue detector signal normally used for automatic gain control (ACC) is fed directly into the analoguedigital converter (ADC) of the separate data logger. We do this to avoid the significant power quantization error $(1 \mathrm{~dB})$ that would be incurred using the link device's own ACC$A D C$ system. The analogue signal was calibrated in an indoor environment using the signal power indication of the indoor unit as a reference. The RAL links were recalibrated by Rutherford Appleton Laboratories shortly before the beginning of the experiment. The calibration curves are shown below in Fig. 2.3, and used to convert the observed voltages to received powers. The transmitted power for all devices was kept constant, but was not separately measured.

\subsubsection{Additional instruments}

To serve as a ground truth, we use OTT Parsivel laser disdrometers (Fig. 2.1c). These can not only measure precipitation intensity but also the size and velocity distributions of 

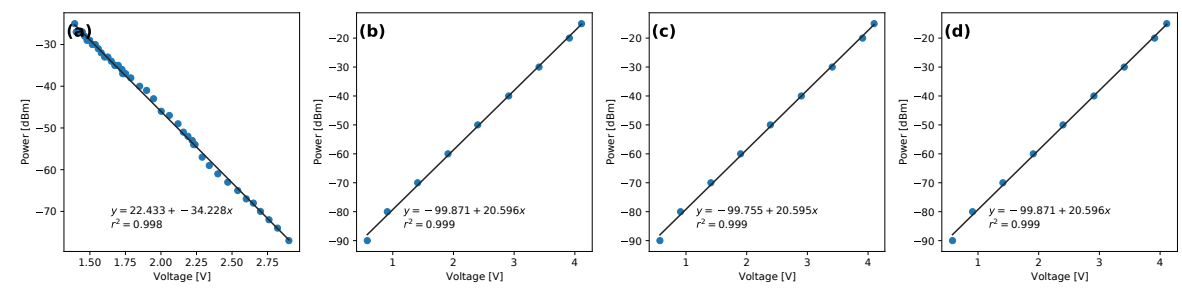

Figure 2.3: Received signal power vs. detector voltage read-out used for the calibration of the detectors. The black line indicates the fitted calibration curve: (a) Nokia, (b) RAL38 GHz horizontal, (c) RAL $38 \mathrm{CHz}$ vertical, and (d) RAL $26 \mathrm{CHz}$ horizontal.

passing precipitation particles over $30 \mathrm{~s}$ intervals. With this information they can provide an approximation of the type of precipitation that occurred. In this manner it is possible to, for example, filter out solid precipitation from the microwave link data or select dry periods to determine the "dry" baseline signal. Due to the small sampling footprint of these devices, they may not give a representative ground truth for the aggregated path measurements. Therefore, five disdrometers are placed at four different locations, spread along the link path (Fig. 2.1a) as evenly as was possible given the urban terrain. At the receiver end of the link path two disdrometers are placed next to each other in close proximity and orthogonal to each other in order to test the accuracy of the disdrometers themselves. All disdrometers are placed on flat or gently sloping rooftops within Wageningen. The disdrometers all contain a built-in preprocessing unit which samples the raw laser amplitude signals, converts them to hydrometeor counts using an algorithm (undisclosed by OTT) based on the principle described in Löffler-Mang and Joss (2000) and aggregates the samples to 30 s intervals. One of the disdrometers at the receiver end had been operational since the beginning of the experiment. It is connected to the same data logger as the link detectors. The other four disdrometers have been operational for a shorter timespan (see Fig. 2.2). They are each connected to a UMTS modem, which relays the disdrometer data to a remote server in real time; see Jaffrain et al. (2011) for more details about these autonomous disdrometer stations.

At the receiver end of the link path an automated tipping bucket rain gauge is placed close to the two disdrometers (Fig. 2.1d), to provide an additional independent measurement. The gauge has a tipping volume of $0.1 \mathrm{~mm}$. Two time-lapse cameras are placed at each end of the link path. On each side one camera is pointed along the path and the other is pointed at the antennas themselves. These serve to allow visual inspection of the link path and the antennas, which can be useful for relating link behaviour to physical events.

For the subsequent data processing we also make use of data from the nearby automatic weather station "Veenkampen" situated roughly $2 \mathrm{~km}$ to the west of Wageningen (operated by the university's Meteorology and Air Quality group) for ambient temperature, relative humidity, wind speed, visibility and pressure measurements. 
Table 2.1: Properties of the link antennas used in this experiment.

\begin{tabular}{llll}
\hline & Nokia & RAL 38 CHz & RAL 26 CHz \\
\hline Antenna diameter & $300 \mathrm{~mm}$ & $150 \mathrm{~mm}$ & $250 \mathrm{~mm}$ \\
Antenna gain & $40.1 \mathrm{dBi}$ & $33.0 \mathrm{dBi}$ & $34.5 \mathrm{dBi}$ \\
Beam width & $1.6^{\circ}$ & $3.5^{\circ}$ & $3.5^{\circ}$ \\
\hline
\end{tabular}

\subsection{Data processing}

\subsubsection{Disdrometers}

\subsubsection{Preprocessing}

The raindrop size and velocity distributions are corrected for known instrumental biases using the method of Raupach and Berne (2015), which involves two steps. Step one is shifting the velocity distributions so that the average velocities per size class match the theoretical terminal velocities for raindrops of that size class. Step two is multiplying the number of detected particles per size class by a class- and rain intensity-dependent correction factor. These correction factors were obtained by Raupach and Berne (2015) from concurrent measurements with a 2-D video disdrometer (2DVD), assuming the 2DVD measurements to be unbiased. Using these corrected distributions we derive rain intensities and other bulk quantities.

Whereas Raupach and Berne (2015) use the theoretical raindrop terminal velocity model of Beard (1977) to determine the bias in velocity distribution we use the model of Beard (1976). The former is a simplification and approximation of the latter, designed to reduce computational expense. However, we found that on a contemporary desktop computer the time needed to compute terminal velocities was negligible using either model. Both models need the ambient pressure and temperature to calculate the raindrop terminal velocity. We used the temperature and pressure measured by the automatic weather station "Veenkampen". As this station is situated outside the built-up area of Wageningen, there might be a slight bias in temperature as compared to the urban areas that the disdrometers are situated in.

The model of Beard (1976) does not compute the terminal velocity directly from only the pressure and temperature but instead needs the density of the water drops and ambient air as well as the surface tension of the air-water interface as input. For the density of water as a function of temperature we use the empirical formula of Kell (e.g. Battan, 1973). For the surface tension of the air-water interface we employ the empirical relation proposed by Vargaftiket al. (1983).

\subsubsection{Derived data}

In the subsequent analysis we compare the attenuation encountered by the microwave link signals with the rainfall along the link path. We also make use of an $R-k$ relation 

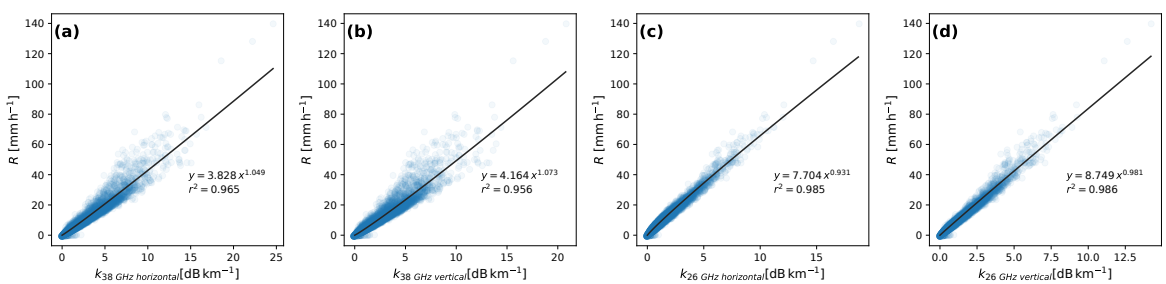

Figure 2.4: Disdrometer-derived rainfall intensities plotted against disdrometer-derived specific attenuation at several frequencies and polarizations of the incident radiation. The black line indicates the fitted curves: (a) $38 \mathrm{CHz}$ horizontal, (b) $38 \mathrm{GHz}$ vertical, (c) $26 \mathrm{CHz}$ horizontal, and (d) $26 \mathrm{CHz}$ vertical.

based on the actual rainfall along the path and the expected attenuation due to this rainfall. In order to do so, we assume that the corrected drop size distributions obtained from the disdrometer stations represent the ground truth for that location. The specific attenuation is derived from the drop size distributions using Eq. (3) and Eq. (4). We derive values for a carrier frequency of both 38 and $26 \mathrm{CHz}$ and for both horizontally and vertically polarized radiation.

We calculate the scattering amplitude matrix for each diameter class using the T-matrix approach developed by Waterman (1965). The computations are done using an algorithm adapted from FORTRAN code developed by Mishchenko et al. (1996); Mishchenko and Travis (1998); Mishchenko (2000) and reimplemented using the Python programming language. As the laser disdrometer cannot provide information on the geometric shape or orientation of the particles, we make use of an orientation averaging scheme. For this purpose we have adapted the particle orientation averaging functions developed by Leinonen (2014) from their T-matrix package. The shape of the raindrops is approximated by an oblate spheroid, with axis ratio dependent on the volume-equivalent diameter. We use the axis ratios suggested by Thurai et al. (2007). The complex index of refraction is needed to calculate the T-matrix. For rain drops we assume the empirically determined formula for the temperature-dependent complex index of refraction for pure liquid water by Liebe et al. (1991) where we use a temperature of $15^{\circ} \mathrm{C}$. Rainfall intensity is calculated with Eq. (2), using the corrected drop size distributions.

In order to provide a comparison for the link measurements, the derived attenuation and rain intensity are then averaged over the link path using a weighted mean over all five disdrometers. For each point along the path, the value of the quantity is taken to be equal to the value derived at the nearest disdrometer. The mean over the path is thus equal to the mean of the disdrometers weighted by the fraction of the path that is closest to that disdrometer. The precipitation type and presence as determined by the Parsivel algorithm are also used. In this case, the path-averaged type is assigned as "mixed" whenever two or more Parsivels register different precipitation types. It is considered "dry" only when all Parsivels agree that there is no precipitation. In all other cases when one or more Par- 
Table 2.2: Coefficients and exponents ( $a$ and $b$ parameters) of the $R-k$ relationship derived from different sources for frequencies of 38 and $26 \mathrm{CHz}$ for both horizontally and vertically polarized radiation. Unit of a is $\mathrm{mm} \mathrm{h}^{-1} \mathrm{~dB}^{-b} \mathrm{~km}^{b}$.

\begin{tabular}{lrrrrrrrr}
\hline & $a_{38 \mathrm{H}}$ & $b_{38 \mathrm{H}}$ & $a_{38 \mathrm{~V}}$ & $b_{38 \mathrm{~V}}$ & $a_{26 \mathrm{H}}$ & $b_{26 \mathrm{H}}$ & $a_{26 \mathrm{~V}}$ & $b_{26 \mathrm{~V}}$ \\
\hline This thesis & 3.83 & 1.05 & 4.16 & 1.07 & 7.70 & 0.93 & 8.75 & 0.98 \\
Leijnse et al. (2010) & 3.35 & 1.02 & 3.70 & 1.05 & 6.72 & 0.91 & 7.79 & 0.95 \\
ITU-R & 2.82 & 1.13 & 3.06 & 1.17 & 5.92 & 1.01 & 6.69 & 1.06 \\
\hline
\end{tabular}

sivels detect precipitation, that precipitation type is assigned as the path-averaged value. We distinguish five broad categories of precipitation: liquid, snow, hail and ice pellets, graupel, and mixed hydrometeors or melting snow. In the subsequent analyses we will mostly be concerned with liquid precipitation, as the other types were rare during the observation period.

\subsubsection{Rainfall intensity-specific attenuation relationship}

The disdrometer-derived rainfall intensities and specific attenuations at the frequencies employed in the microwave links are plotted with respect to each other in Fig. 2.4. Each dot represents a single $30 \mathrm{~s}$ DSD measurement (not path-averaged) from an individual disdrometer. Measurements from all five disdrometers were used. Only data points that were characterized as liquid precipitation by the Parsivel algorithm and where rainfall intensity was higher than $0.1 \mathrm{~mm} \mathrm{~h}^{-1}$ were selected. These data were then used to fit $R-k$ power-law models using a non-linear least-squares algorithm. Coodness-of-fit for these relationships is very high: $r^{2}=0.956$ to $r^{2}=0.986$. Also note that the power-law exponents are all close to one, indicating that specific attenuation and rainfall intensity are nearly proportional to each other at the employed frequencies. These relationships are then applied to the specific attenuations measured with the links.

In Table 2, the determined values are compared with others found in the literature. The values found by Leijnse et al. (2010) were based on drop size distributions collected in the Netherlands as well, but were collected using filter-paper in 1968 (Wessels, 1972). We also compare with the formal ITU (International Telecommunication Union) recommendation regarding the modelling of microwave attenuation due to rain (ITU, 2005). We see that the exponents $(b)$ are very similar for the relationships obtained in this work and those obtained by Leijnse et al. (2010) and the coefficients (a) found by Leijnse et al. (2010) are 11 to $13 \%$ lower than those found here. We can also conclude that the $a$ parameter is too low ( 23 to $26 \%$ ) and the $b$ parameter is too high ( 8 to $9 \%$ ) in the ITU recommendation with respect to our study for the Dutch rainfall climatology. For the analyses in Sect. 2.5 we have used these locally derived power laws where applicable. 


\subsubsection{Microwave links}

In order to calculate the rainfall intensities, the attenuation caused by rainfall and the attenuation caused by other atmospheric effects must be distinguished. Rahimietal. (2003) proposed a two-step approach in order to do so.

The first step is to determine which of the sampled periods are dry. Overeem et al. (2013) uses the assumption of spatial correlation of rainfall to determine "wet" and "dry" periods for microwave links in cellular communication networks. In short, a period is considered "wet" if nearby links show a mutual decrease in received signal levels. As we are considering only a single path, such a method would not be applicable here. An alternative is to use the assumption of temporal correlation of rainfall. Schleiss and Berne (2010) suggest using a moving window standard deviation threshold. Similarly, Chwala et al. (2012) used a Fourier-transform based method to distinguish between wet and dry spells. Other methods applicable to a single link path are e.g. a Markov switching algorithm (Wang et al., 2012) and the use of dual-frequency links (Rahimi et al., 2003). Here, the path-aggregated disdrometer data is used to determine dry periods independently of the microwave link data.

The second step in the algorithm is to determine a suitable baseline signal level using the selected dry periods. The implemented baseline algorithm uses a rolling median over all measurements classified as dry in the surrounding centred $24 \mathrm{~h}$ period to determine the baseline signal for each time step. The specific attenuation is then calculated as

$$
k=\max \left(\frac{P_{\mathrm{ref}}-P}{L}, 0\right),
$$

where $P$ is the received power and $L$ is the path length. Rainfall intensity is derived from the corrected attenuation using the power-law relationship of Eq. (5). The parameters $a$ and $b$ in this equation are obtained from the disdrometer data as described in Sect. 2.4.1.3.

Furthermore, the rainfall intensity is set to 0 when the disdrometer indicates dry weather. Note that we do not perform any a priori additional corrections on the microwave link rainfall estimate, such as correcting for wet antenna attenuations. The goal is, after all, to use this basic estimate to assess potential error inducing phenomena, not to evaluate a best-effort estimation.

\subsection{Results and discussion}

\subsubsection{Overview}

In the following section, we use the rainfall intensity as measured by the Parsivel disdrometers as a reference to assess the link-derived rainfall. Unless stated otherwise, we 
use the corrected DSD-derived rainfall intensities, not the rain intensities that the internal Parsivel algorithm produces.

In order to better understand the different phenomena that contribute to the microwave link attenuation signal, we present a number of illustrative events from the dataset. We search for events that can be related to a single type of attenuating phenomenon in order to gain insight into the separate phenomena. We will first analyse the performance of the simple algorithm for measuring liquid precipitation and take a quick look at solid and mixed precipitation. We will then show how temperature and wet antennas, e.g. caused by dew formation affect the signal. Finally, we will look at some currently unexplained phenomena and also give some examples where different phenomena occur simultaneously. All times in the description of the events are given in UTC.

\subsubsection{Rainfall events}

We compare the link-derived rainfall rates using the simple algorithm (excluding any specific corrections) described in Sect. 2.4.2 with the spatially averaged rainfall rates derived from the disdrometers using the corrected DSDs. To assess the reliability of the disdrometer measurements as a ground truth, we first compare the collocated disdrometers with the tipping bucket rain gauge and each other. We used data of the entire measurement period where rain intensities higher than $0.1 \mathrm{~mm} \mathrm{~h}^{-1}$ were registered. We find that the correlations of the disdrometers with the rain gauge ( $r=0.928$ and $r=0.927$ ) were only slightly lower than the correlation of the disdrometers with each other $(r=$ 0.959). The mean differences between the disdrometers and the rain gauge were 0.039 and $0.129 \mathrm{~mm} \mathrm{~h}^{-1}$, respectively, while the mean difference between both disdrometers amounted to $0.074 \mathrm{~mm} \mathrm{~h}^{-1}$. That means that the disdrometers slightly overestimate the rain intensities as compared to the rain gauge, but this is of the same order of magnitude as the differences between the identical collocated disdrometers. Therefore, we will assume the path-averaged disdrometer measurements to be the "true" path-averaged rainfall for the purpose of evaluating the link measurements.

Figure 2.5 shows an example of a single short isolated rain event on 14 July 2015 as indicated by the disdrometers. We chose this example because there are no attenuating phenomena contributing to the dynamics of the signal other than rain in this event. Note that the received signal level of the Nokia is offset by $14 \mathrm{~dB}$ in order to fit into the plot. This is done consistently for all following figures. As the received power level can vary within the dry periods that we use to determine the baseline power level, we also indicate the 95th and 5th percentile of the received power level over the dry intervals within the surrounding 24-h moving window. This gives an indication of the variability of the baseline power level. Thus, if the rain-induced attenuation is within this range, it cannot be distinguished from variability in the baseline without further processing. The event consists of two distinct small peaks. The first peak of the path-average rainfall intensity only reaches $0.7 \mathrm{~mm} \mathrm{~h}^{-1}$, while the second peak reaches $8 \mathrm{~mm} \mathrm{~h}^{-1}$. We see that the second peak causes a clear attenuation of the received signal level of all the links. The smaller peak in rain intensity causes only a small attenuation in the $38 \mathrm{CHz}$ links, fully within the 95th 
and 5th percentile range of the dry signal. Although the presence of rainfall is detected unambiguously by all the instruments, the magnitude of the response differs between the links. Both the horizontally and vertically polarized detectors in the $38 \mathrm{CHz} \mathrm{RAL} \mathrm{link}$ give very similar responses, which is expected as they receive different components of the same signal and also share a substantial part of their electric signal path, including the antenna itself. Most notable is the difference between the signal of the Nokia link and the RAL link operating at (nearly) the same frequency and polarization. Although the magnitudes are similar, the Nokia link has far less variability of the baseline signal level than all other link instruments. This difference could be caused by the differences in internal electronics of the detector. Another point of interest is that using the median of all dry data points in the $24 \mathrm{~h}$ period, our estimation of the baseline signal level of the $26 \mathrm{CHz}$ RAL link and to a lesser extend the $38 \mathrm{CHz}$ RAL link is too high, resulting in an additive overestimation of the rain intensity. The calculated apparent rainfall during the first peak is completely below this line, indicating that this can be regarded as noise. Regardless, the peak rainfall estimate from the Nokia link is very close to the disdrometer estimate. We can also see that attenuation of the microwave link signal persists for several minutes after the end of the rainfall event (according to the disdrometers) and slowly decays during this time. This could be the consequence of the link antennas becoming wet due to the rain and subsequently drying up after the event (Minda and Nakamura, 2005; Leijnse et al., 2008). In Fig. 2.5a the received signal level of the near-infrared link is also plotted. Attenuation of this signal is indicative of visibility. In this case the visibility loss is highly correlated with rain (correlation coefficient $r=-0.86$ ).

We illustrate the response of the link signals to rain with two more example events of a longer duration. One low-intensity drizzle event and one higher-intensity convective rain event with some spatial heterogeneity. On both occasions we use only the times for which at least one of the disdrometers indicate rain has occurred for further analyses.

The first event, on 24 November 2015, consists of a low-intensity drizzle period (intensities under $2 \mathrm{~mm} \mathrm{~h}^{-1}$ for most of the event) that persists for around $12 \mathrm{~h}$. The course of the event is illustrated in Fig. 2.6. The RAL links show some variability in the baseline power level, varying over a range of 0.11 to $0.14 \mathrm{~dB}$ over the course of the event until 13:00 UTC. The Nokia link, in contrast, stays remarkably stable during the entire event, ranging only $0.02 \mathrm{~dB}$ over the same period. After that, all links show a large drop in baseline power level, with the largest magnitude in the RAL $38 \mathrm{CHz}$ link $(3.46 \mathrm{~dB})$ and the smallest in the Nokia link $(0.33 \mathrm{~dB})$. There is a fairly strong correlation of link derived rain intensity with disdrometer rain intensity for both the Nokia link and the $26 \mathrm{CHz}$ RAL link $(r=0.870$ and $r=0.854$, respectively) and less so for the $38 \mathrm{CHz}$ RAL link ( $r=0.594$ for vertical polarization and $r=0.232$ for horizontal polarization).

We estimate the additive and multiplicative bias in the link-derived rain intensity by the parameters of a simple linear regression with the spatial average of the disdrometer derived rainfall, which is illustrated in Fig. 2.7a-d. All microwave links overestimate the rain intensity to some extent. Additive bias of the RAL links is between 2.2 and $3.7 \mathrm{~mm}$, which is more than the actual rainfall during most of the event. Additive bias is lowest in the 


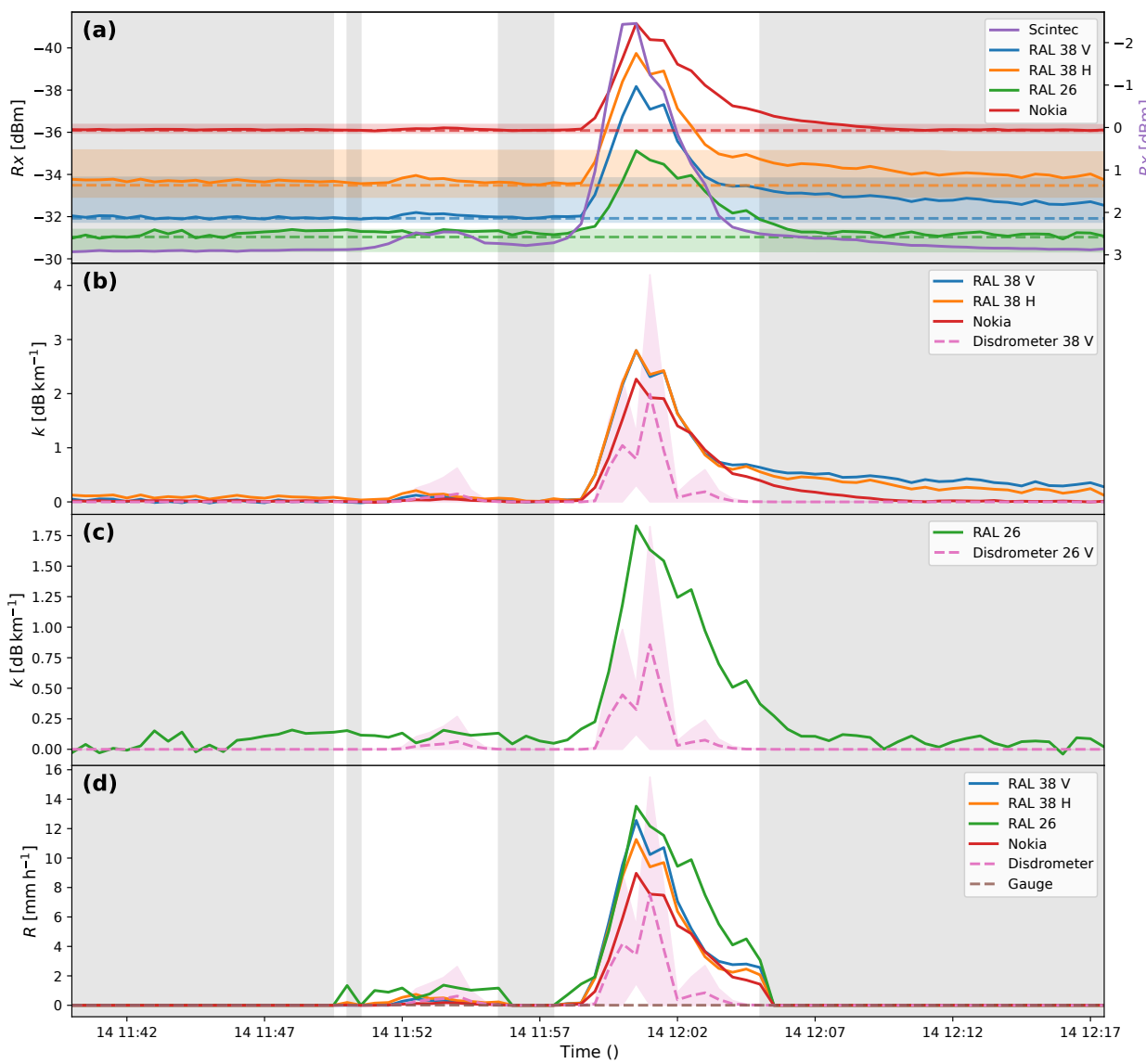

Figure 2.5: Time series of an event on 14 July 2015. (a) Received power levels (solid lines) and referencelevels (median overdry periods in a 24 h moving window: dashed lines). The 5 th and 95 th percentile power level over dry periods in a $24 \mathrm{~h}$ moving window are indicated by the coloured shading. (b) Specific attenuation of the $38 \mathrm{CHz}$ links derived using the reference levels as well as the theoretical specific attenuation at $38 \mathrm{GHz}$ derived from the disdrometers. Both the weighted spatial average (dashed line) and weighted spatial standard deviation (shaded area) are shown. Panel (c) is the same as (b), but for $26 \mathrm{CHz}$. (d) Rainfall intensities derived from the link attenuations using the $R-k$ power law and rainfall intensities derived from the disdrometers. Both the weighted spatial average (dashed line) and weighted spatial standard deviation (shaded area) are indicated. The rainfall intensities derived from the tipping bucket gauge are indicated with the brown dashed line. Dry periods, as determined with the disdrometers, are represented by grey shaded areas. 
Nokia link-derived data, which seems to be in line with the very stable baseline. However, it is still $0.8 \mathrm{~mm} \mathrm{~h}^{-1}$, which is a problem for accurately measuring accumulations from light rain events. We can also see that in this case visibility cannot be reliably used as a proxy for rainfall intensity, as is most clearly seen after 13:00.

The second event, on 4 November 2015, is more spatially heterogeneous ( $C V=0.64$, as opposed to $\mathrm{CV}=0.50 \mathrm{in}$ the previous event.). The higher spatial heterogeneity and higher rainfall intensities suggest a convective rainfall event. The total event lasts for $8 \mathrm{~h}$ (see Fig. 2.8). Peaks in spatially averaged rainfall intensity during this event are on the order of 20 to $30 \mathrm{~mm} \mathrm{~h}^{-1}$, and individual disdrometer measurements reach up to $55 \mathrm{~mm} \mathrm{~h}^{-1}$. Once again the baseline of the Nokia link is remarkably stable (range $=0.19 \mathrm{~dB})$, similar to the $26 \mathrm{CHz}$ RAL link (range $=0.33 \mathrm{~dB}$ ), while the $38 \mathrm{CHz}$ RAL link has a highly variable baseline (range $=1.53 \mathrm{~dB}$ for the horizontally polarized signal and $3.96 \mathrm{~dB}$ for the vertically polarized signal). During most of this event visibility seems to be a reasonable proxy for the rainfall intensity. Correlations of link-derived rainfall with disdrometer-derived rainfall are much higher overall ( $r=0.90$ to $r=0.93$ ) (Fig. 2.7e-h) than for the event on 24 November 2015. Additive bias is of the same order of magnitude as for the drizzle case, which means that the additive bias relative to the rainfall intensities is much less for this event than for the drizzle event. Multiplicative bias varies from a factor of 1.4 to 1.6.

We now compare link derived rain intensity with disdrometers derived rain intensity for the entire measurement period; results are shown in Fig. 2.7i-I. Data points where the path-average rainfall intensity derived from the disdrometer measurements are less than $0.1 \mathrm{~mm} \mathrm{~h}^{-1}$ or at least one of the disdrometers indicate the presence of solid precipitation are excluded. We also exclude the period during which the link transmitters were not functioning. The Nokia link performs better than the RAL links in terms of correlations. In all cases the links significantly overestimate the rainfall intensity, both in an additive (regression intercept ranging from 0.6 to $2.2 \mathrm{~mm} \mathrm{~h}^{-1}$ ) and a multiplicative (regression slope ranging from 1.5 to 1.7) sense. The general overestimation could be attributed to attenuating phenomena other than rain being erroneously processed as rain in the basic algorithm, in part due to the simple baseline determination process not taking these into account and because e.g. no correction was applied for wet antennas. A similar regression in terms of specific attenuations produces nearly identical results. Therefore, we conclude that uncertainties in the $R-k$ relation do not significantly explain uncertainties in the rainfall estimation.

In typical operational settings, longer temporal measurement intervals such as $15 \mathrm{~min}$ are common (e.g. Overeem et al., 2016a). In order to illustrate the performance of a basic algorithm without any sort of correction at this resolution, Fig. $2.7 \mathrm{~m}-\mathrm{p}$ show the scatter plot and linear regression for the entire dataset, but down-sampled using a 15 min mean. The correlation for the Nokia link is slightly higher with the 15 min intervals than it is using 30 s intervals and the scatter around the regression line is lower. In the case of the RAL links, the performance is better for the $15 \mathrm{~min}$ accumulations than for the $30 \mathrm{~s}$ intervals in terms of correlation and scatter, but worse when considering bias. 


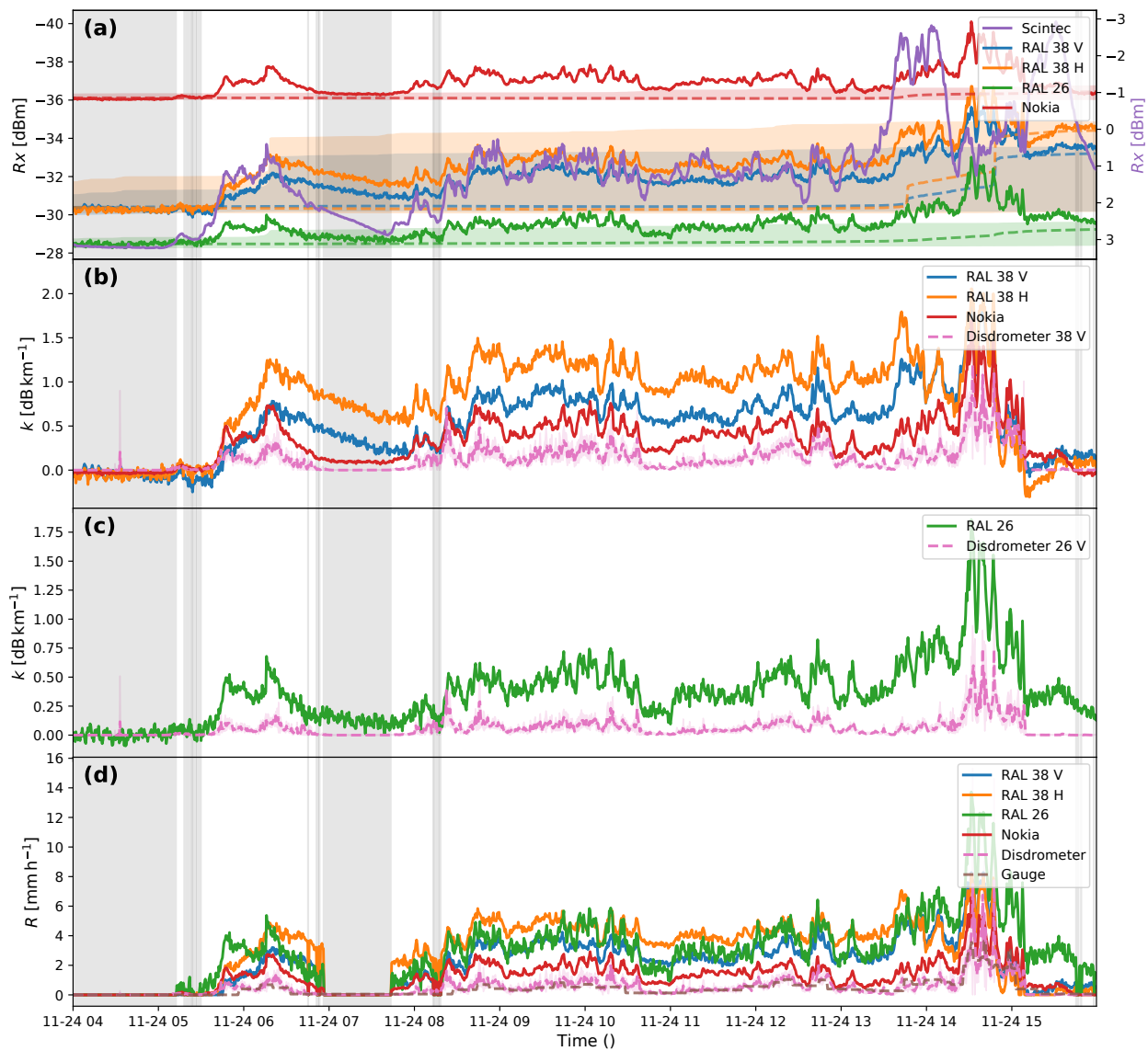

Figure 2.6: Time series of an event on 24 November 2015. (a) Received power levels (solid lines) and reference levels (median over dry periods in a $24 \mathrm{~h}$ moving window: dashed lines). The 5th and 95 th percentile power levels over dry periods in a $24 \mathrm{~h}$ moving window are indicated by the coloured shading. (b) Specific attenuation of the $38 \mathrm{CHz}$ links derived using the reference levels as well as the theoretical specific attenuation at $38 \mathrm{GHz}$ derived from the disdrometers. Both the weighted spatial average (dashed line) and weighted spatial standard deviation (shaded area) are shown. Panel (c) is the same as (b), but for $26 \mathrm{CHz}$. (d) Rainfall intensities derived from the linkattenuations using the $R-k$ power law and rainfall intensities derived from the disdrometers. Both the weighted spatial average (dashed line) and weighted spatial standard deviation (shaded area) are shown. The rainfall intensities derived from the tipping bucket gauge are indicated with the brown dashed line. Dry periods, as determined with the disdrometers, are represented by grey shaded areas. 

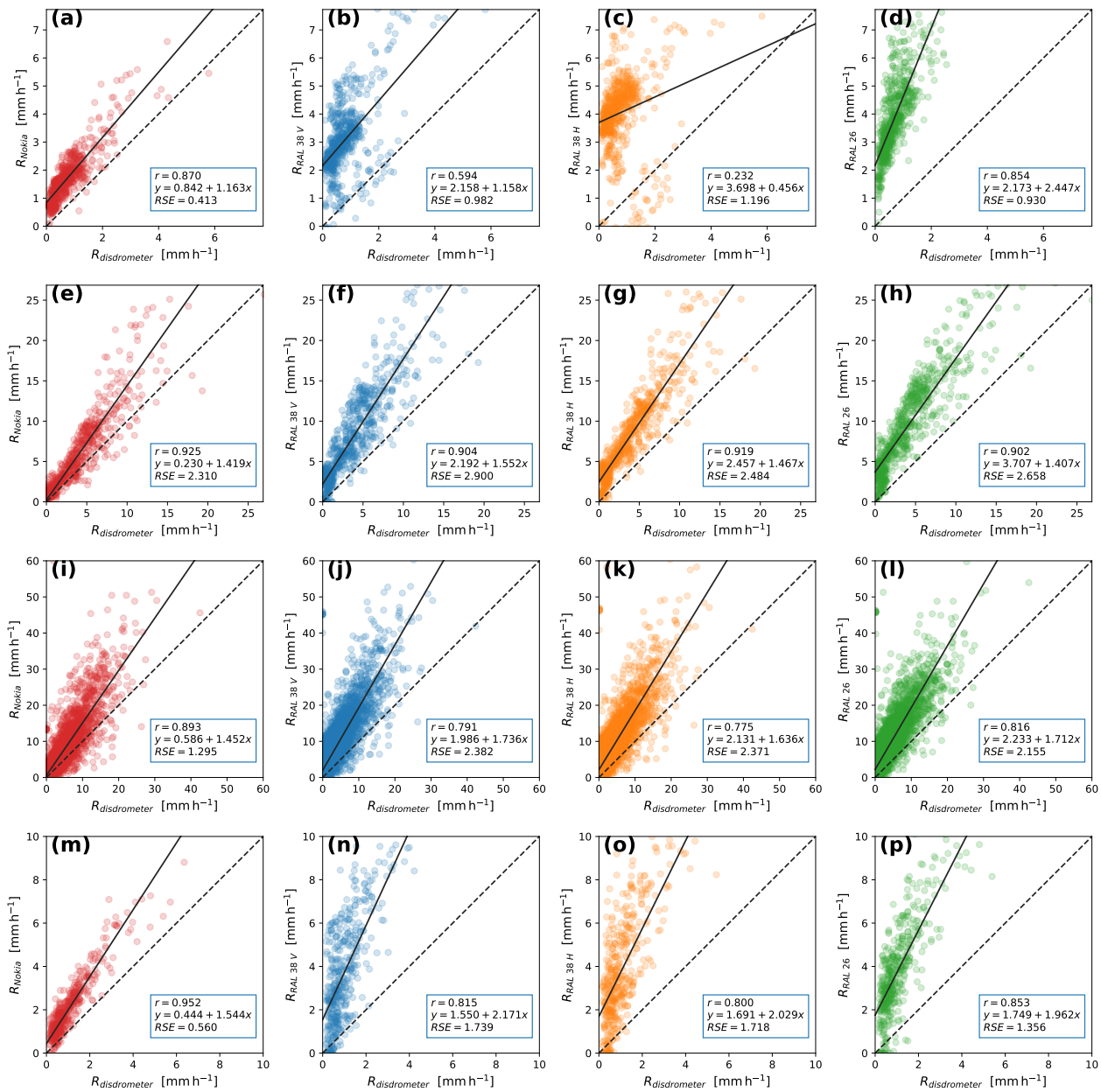

Figure 2.7: Scatterplots of link-derived rainfall intensities vs. disdrometer-derived rainfall intensities. Solid lines indicate a linear least-squares fit, dotted lines indicate the $1: 1$ line. Within each plot the correlation coefficient $(r)$, the fitted line function and the residual standard error (RSE) are also shown. Links from left to right: Nokia, RAL $38 \mathrm{CHz}$ vertical, RAL $38 \mathrm{GHz}$ horizontal, and RAL $26 \mathrm{CHz}$. From top to bottom: 24 November 2015 (down-sampled to 30 s), 4 November 2015 (down-sampled to $30 \mathrm{~s}$ ), whole dataset down-sampled to $30 \mathrm{~s}$, and whole dataset down-sampled to 15 min. 

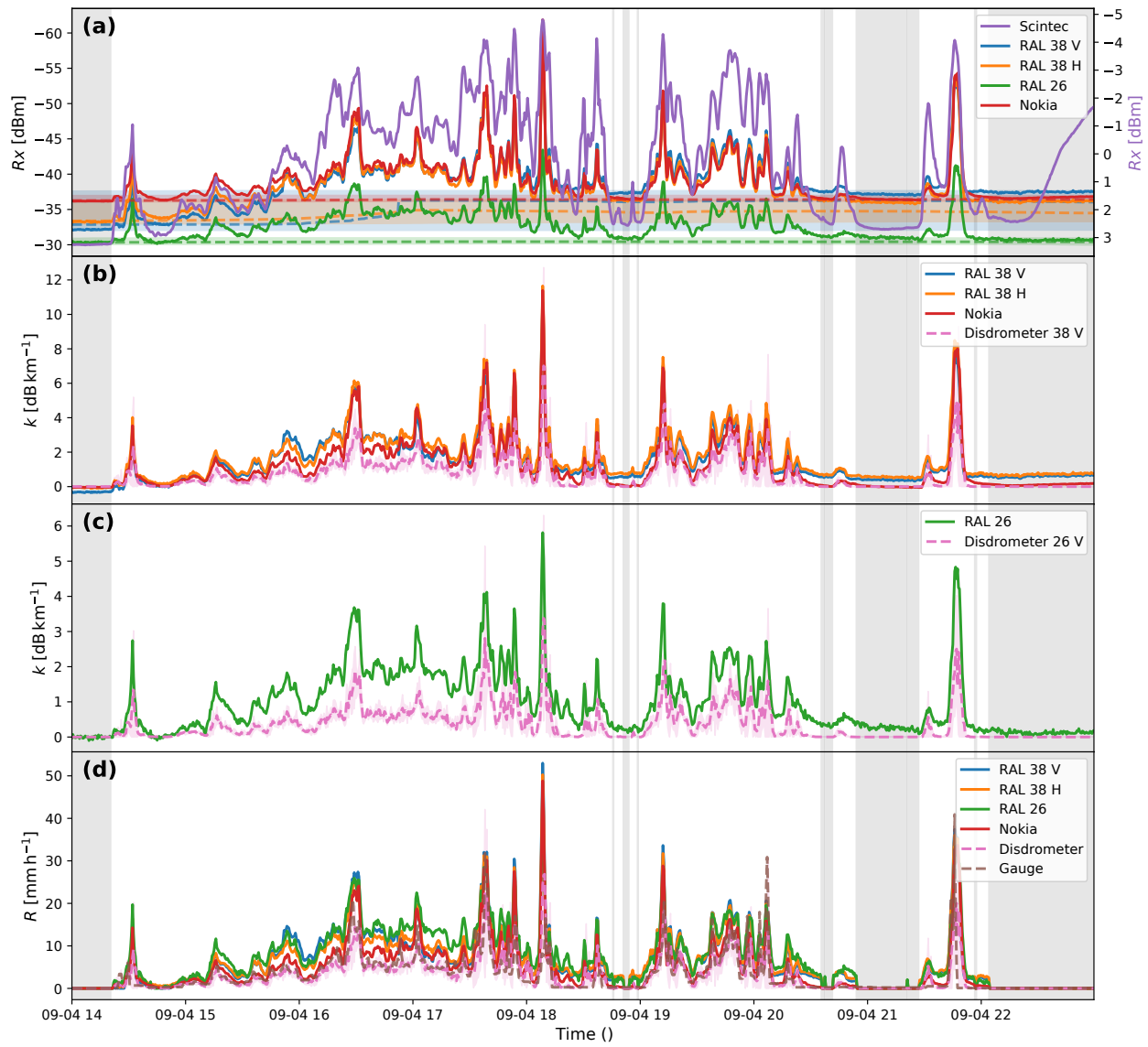

Figure 2.8: Time series of an event on 4 November 2015. (a) Received power levels (solid lines) and reference levels (median over dry periods in a $24 \mathrm{~h}$ moving window: dashed lines). The 5th and 95th percentile power level over dry periods in a $24 \mathrm{~h}$ moving window are indicated by the coloured shading. (b) Specific attenuation of the $38 \mathrm{CHz}$ links derived using the reference levels as well as the theoretical specific attenuation at $38 \mathrm{GHz}$ derived from the disdrometers. Both the weighted spatial average (dashed line) and weighted spatial standard deviation (shaded area) are shown. Panel (c) is the same as (b), but for $26 \mathrm{CHz}$. (d) Rainfall intensities derived from the linkattenuations using the $R-k$ power law and rainfall intensities derived from the disdrometers. Both the weighted spatial average (dashed line) and weighted spatial standard deviation (shaded area) are shown. The rainfall intensities derived from the tipping bucket gauge are indicated with the brown dashed line. Dry periods, as determined with the disdrometers, are represented by grey shaded areas. 


\subsubsection{Solid/mixed precipitation}

Very few solid or mixed precipitation events occurred during our measurement period. Figure 2.9 shows one of the few snowfall occurrences during the campaign, on 4 February 2015 . At this point in the campaign only one disdrometer was yet placed and no rain gauge was available, which limits the potential for a quantitative comparison. Figure 2.9d shows time-lapse camera footage taken during different stages of this event. The background shades in Fig. 2.9b indicate the type of precipitation as indicated by the Parsivel internal algorithm (blue is liquid precipitation, green is snow, red is mixed precipitation). The total event duration is about $40 \mathrm{~min}$, yet the event is quite variable in time. As indicated by the background colours and the camera footage, this short event starts out with a mixture of rain and ice pellets and then turns into snowfall. Along with the mixed precipitation the temperature drops from 4 to $1{ }^{\circ} \mathrm{C}$. During the snowfall, the temperature drops further to $0{ }^{\circ} \mathrm{C}$. The absolute values of the disdrometer-derived precipitation intensity cannot be taken at face value here, as our processing algorithm treats every particle as a raindrop. This results in far too high values during snowfall, as we do not account for the lower density of a typical snowflake. As the disdrometer rainfall intensity shown here is that of only one disdrometer and as it was placed at one far end of the link path, we do not expect the small-scale variations to match exactly with those of the link attenuation. However, the overall dynamics of the intensity and the type of precipitation can provide some useful information.

Between 15:10 and 15:30, the links are attenuated with a magnitude that corresponds roughly with the precipitation intensity measured by the disdrometer, assuming that it is pure rain. Afterwards, when snow starts to fall between 15:30 and 15:55, the precipitation intensity derived from the disdrometers becomes a factor of 10 higher than the link-derived precipitation intensity, but this is likely to be due to the faulty disdrometer algorithm when applied in snow. While both the disdrometer and the camera footage seem to indicate that the precipitation stops after 15:55, the attenuation of the links persists until the signal level returns to its initial value between 16:00 and 16:15. At this point the temperature hovers at a few tenths of degrees above zero, and the camera footage indicates some residual snow is left on the antenna covers. The snow deposits are mostly on top of the covers and is mostly still present by 16:17, when attenuation has decayed fully, so snow deposits alone cannot explain the persistent attenuation. Based on the above observations a possible explanation of the persistent attenuation effect would be the partial melting of residual snow on top of the antenna cover, which then keeps the antenna cover wet. However, the available data is not sufficient to confirm this.

Because there were few snowfall events during the entire campaign period and each of them was of short duration and mixed with other types of precipitation (similar to the event described in this section), no meaningful analyses could be done regarding the relationship between attenuation and snowfall intensity. 

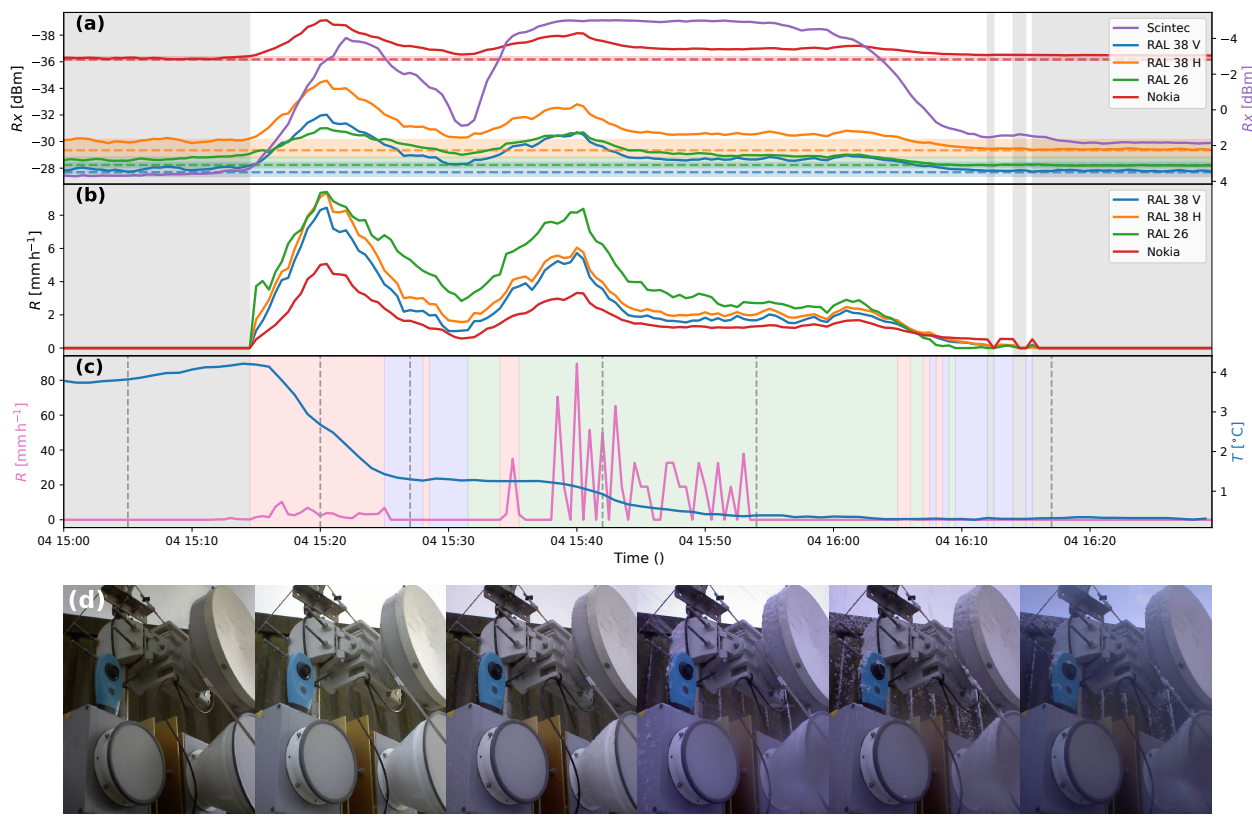

Figure 2.9: Time series of an event on 4 February 2015. (a) Received power levels at the detectors (solid lines) and reference levels (median over dry periods in a $24 \mathrm{~h}$ moving window: dashed lines). The 5th and 95th percentile power levels over dry periods in a $24 \mathrm{~h}$ moving window are indicated by the coloured shading. (b) Derived rainfall intensities using the basic algorithm. (c) Rainfall intensities derived from the disdrometer positioned at "Forum" and ambient air temperature at $2 \mathrm{~m}$ at the "Veenkampen" meteorological station. Dry periods, as determined with the disdrometers, are represented by greyshaded areas. Periods with mixed precipitation are indicated with red shaded areas; periods where only liquid precipitation is detected are indicated in blue; and periods with snow are indicated in green. (d) Images from the time-lapse camera at the location of the transmitting antennas aimed at the antennas. The times at which these images were capture are indicated by the vertical dashed lines in (c). 


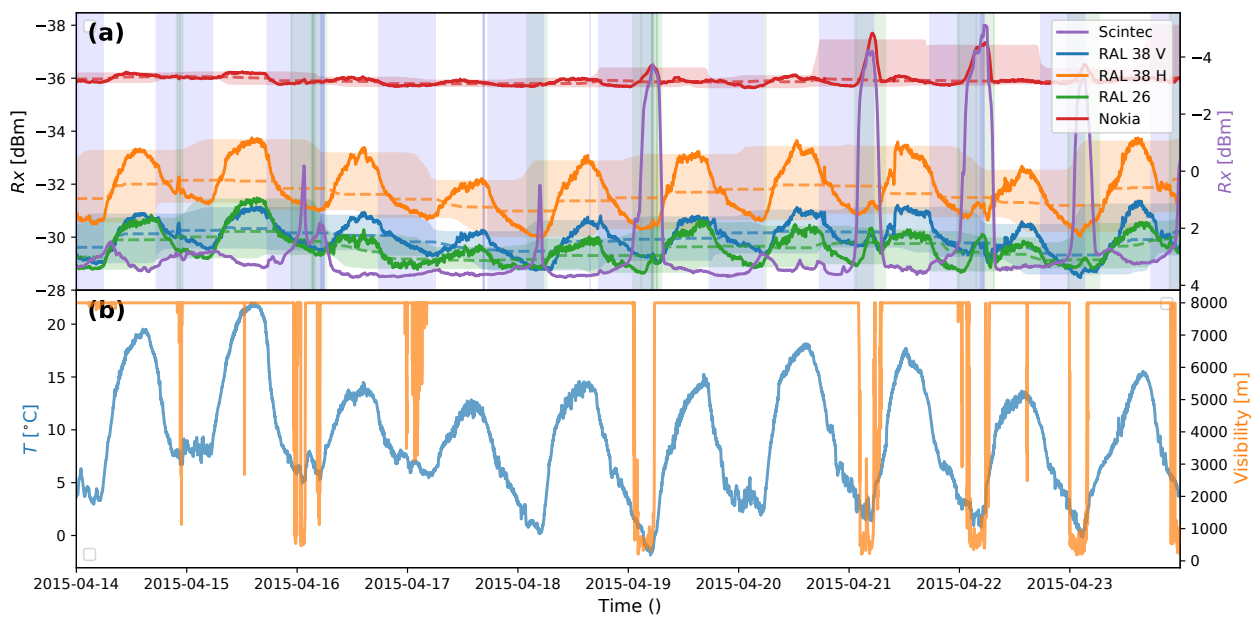

Figure 2.10: Time series of the period between 14 April 2015 and 24 April 2015. (a) Received power levels (solid lines) and reference levels (median over dry periods in a $24 \mathrm{~h}$ moving window: dashed lines). Periods with a negative net radiation flux at the surface are indicated with blue shading. Periods with a relative humidity $>90 \%$ are indicated with green shading. (b) Several atmospheric variables measured at the "Veenkampen" meteorological station: visibility and ambient air temperature at $2 \mathrm{~m}$ indicated with orange and blue lines, respectively.

\subsubsection{Temperature}

Throughout the entire observation period a diurnal oscillation can be seen in the attenuation signal. This diurnal cycle is present in all signals, although the magnitude of the oscillation is in general significantly higher for the RAL links (1.0-1.5 dB) than it is for the Nokia link $(\sim 0.2 \mathrm{~dB})$. The magnitude of the oscillation also varies throughout the observation period. This behaviour does not correspond to any precipitation pattern but seems to follow the known diurnal variations in temperature. Although this pattern can be seen throughout the observational period, the correlation with temperature is not always clear, because the signal is generally much weaker when other attenuating phenomena are present.

Therefore, we will first focus on a relatively long dry period between 14 and 24 April 2015, as shown in Fig. 2.10. During this period the disdrometers picked up no precipitation; however, the received signal levels are not constant. Instead, variations up to $1 \mathrm{~dB}$ are present. In Fig. 2.10b, the time series of ambient air temperature measured by the nearby weather station is plotted for the same period together with the visibility measured at that same station, while in Fig. 2.11, the power levels for this period are plotted against the temperature with a simple linear regression. We performed separate regressions for instances where humidity was above $90 \%$ and for instances where relative humidity was below $90 \%$. This was done to distinguish instances where dew formation on the anten- 

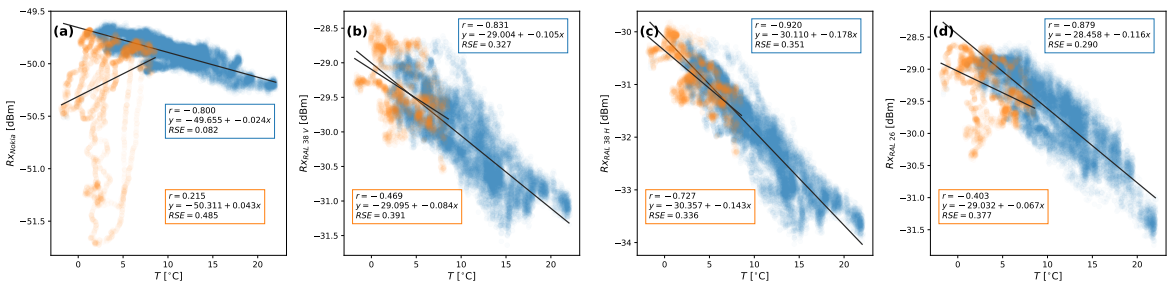

Figure 2.11: Scatterplots of link received power vs. ambient air temperature measured at the "Veenkampen" meteorological station. Blue dots indicate times when relative humidity (as measured at "Veenkampen") is $<90 \%$; orange dots indicate times when relative humidity $>90 \%$. Solid lines indicate linear least-squares regression fit. Links: (a) Nokia Flexihopper, (b) RAL $38 \mathrm{CHz}$ vertical, (c) RAL $38 \mathrm{CHz}$ horizontal, and (d) RAL $26 \mathrm{CHz}$.

nas might have occurred, which we will discuss in the next subsection. In Fig. 2.10a we have also indicated the periods where relative humidity is above $90 \%$ (green shade) and the periods where the net radiation flux towards the surface is negative (blue shade).

There is a strong negative correlation between received power and temperature for all link instruments ( $r=0.80$ to $r=0.92$ ), when relative humidity is below $90 \%$. However, the slope of the linear fit is much lower for the Nokia link $\left(-0.024 \mathrm{~dB} \mathrm{~K}^{-1}\right)$ than for the others (between -0.1 and $-0.2 \mathrm{~dB} \mathrm{~K}^{-1}$ ), even though the Nokia link operates at (nearly) the same frequency and polarization as one of the RAL links. Apparently, the magnitude of the temperature dependence is far more specific to the link hardware, than to the carrier frequency or polarization. The negative correlation between temperature and received power in the RAL devices is much milder when the relative humidity is above $90 \%$ ( $r=-0.40$ to $r=-0.72$ ). For the Nokia link, the temperature dependence disappears at high humidity. This phenomenon is probably related to dew formation at the antennas as is discussed in more detail in Sect. 2.5.5.

In subsequent analyses we expand our investigation of a possible linear temperature dependency to the entire experimental period. However, we exclude two time frames from this analysis. Firstly, the period between 6 and 25 August 2015 when the transmitters were not functioning (but the receivers were). Secondly, we also exclude a period between 11 and 19 May 2015 because a metal construction crane was positioned in the line of sight between the transmitter and receiver (see Sect. 2.5.7) several times in this period. The correlations and regression slopes found for this extended period are shown in Table 2.3 as "whole period".

We consider that dew-related wetting of antennas causes attenuation of the link signal, which muddles the observed temperature dependency. Furthermore, this phenomenon seems only to occur when the nearby weather station registers a relative humidity above $90 \%$. Therefore, we filter the dataset in two more ways in order to separate temperature effects from signal attenuation in the full time period. First, we remove all data points 
Table 2.3: Results of the regression of Fig. 2.11 applied to different subsets of the data.

\begin{tabular}{lrrrrrrrr}
\hline & $\begin{array}{c}\text { Corr. } \\
\text { Nokia }\end{array}$ & $\begin{array}{c}\text { Slope } \\
\text { Nokia }\end{array}$ & $\begin{array}{c}\text { Corr. } \\
\text { RAL 38V }\end{array}$ & $\begin{array}{c}\text { Slope } \\
\text { RAL 38V }\end{array}$ & $\begin{array}{r}\text { Corr. } \\
\text { RAL 38H }\end{array}$ & $\begin{array}{c}\text { Slope } \\
\text { RAL38H }\end{array}$ & $\begin{array}{r}\text { Corr. } \\
\text { RAL 26 }\end{array}$ & $\begin{array}{c}\text { Slope } \\
\text { RAL 26 }\end{array}$ \\
\hline 14-24 April & -0.800 & -0.024 & -0.831 & -0.105 & -0.920 & -0.178 & -0.879 & -0.116 \\
Whole set & 0.019 & -0.003 & -0.461 & -0.153 & -0.565 & -0.179 & -0.546 & -0.113 \\
Rain only & 0.011 & 0.004 & -0.332 & -0.170 & -0.342 & -0.170 & -0.408 & -0.123 \\
Dry only & -0.072 & -0.001 & -0.573 & -0.168 & -0.716 & -0.197 & -0.719 & -0.134 \\
\hline
\end{tabular}

where any disdrometer indicates any form of precipitation. Second, we remove all data points where relative humidity was above $90 \%$. We then find the correlations and slopes indicated in Table 2.3 as "dry only". Furthermore, for completeness we also show correlations and slopes for a subset where, instead of the two abovementioned filters, we apply a filter that only includes periods where any of the disdrometers registered rain (shown in Table 2.3 as "rain only").

The slopes of the linear regression for the RAL links are similar for all data selections (difference within $\pm 15 \%$ compared to the slopes found for 14-24 April), while the correlation coefficients become progressively smaller for the "dry only" selection ( $r=-0.57$ to $r=-0.72$ ), the "whole set" selection ( $r=-0.46$ to $r=-0.57$ ) and the "rain only" selection ( $r=-0.33$ to $r=-0.41$ ). The latter is still surprisingly high considering that the received signal level in this selection includes attenuation by rain. For the Nokia link no significant correlation of received power level to temperature is found for any of the data selections, even though one would expect it based on the findings from 14-24 April.

\subsubsection{Dew and fog}

There is also another phenomenon apparent in Fig. 2.10, especially noticeable in the Nokia link: some sharp drops in received power that evolve from midnight until the early morning ( 00:00-05:00) and then quickly disappear again within $2 \mathrm{~h}$ with peaks of 1 to $2 \mathrm{~dB}$ (see Fig. 2.10a). Comparing to Fig. 2.10b, it is clear that they do not coincide with any change in temperature. Instead, these peaks only appear in periods when the net radiation is negative and the relative humidity is above $90 \%$. The power gradually returns again to the previous level when the net radiation becomes positive and the event is over as soon as the relative humidity drops below $90 \%$. From Fig. 2.10 we can see that these instances (where humidity is above $90 \%$ ) are not correlated to temperature. These characteristics indicate that dew formation on the antennas is a plausible explanation for this phenomenon. The hypothesis is as follows: relative humidity in the air approaching $100 \%$ and a net loss of radiative energy at the surface are indicative of dew formation; water condenses on the antenna covers and builds up a thin layer of water which causes attenuation proportional to the thickness of the layer (see Leijnse et al., 2008); as the net radiative flux changes sign and the water layer dries up, the attenuation slowly returns to the baseline level.

Fog and dew often occur under the same conditions and it is thus difficult to rule out fog 

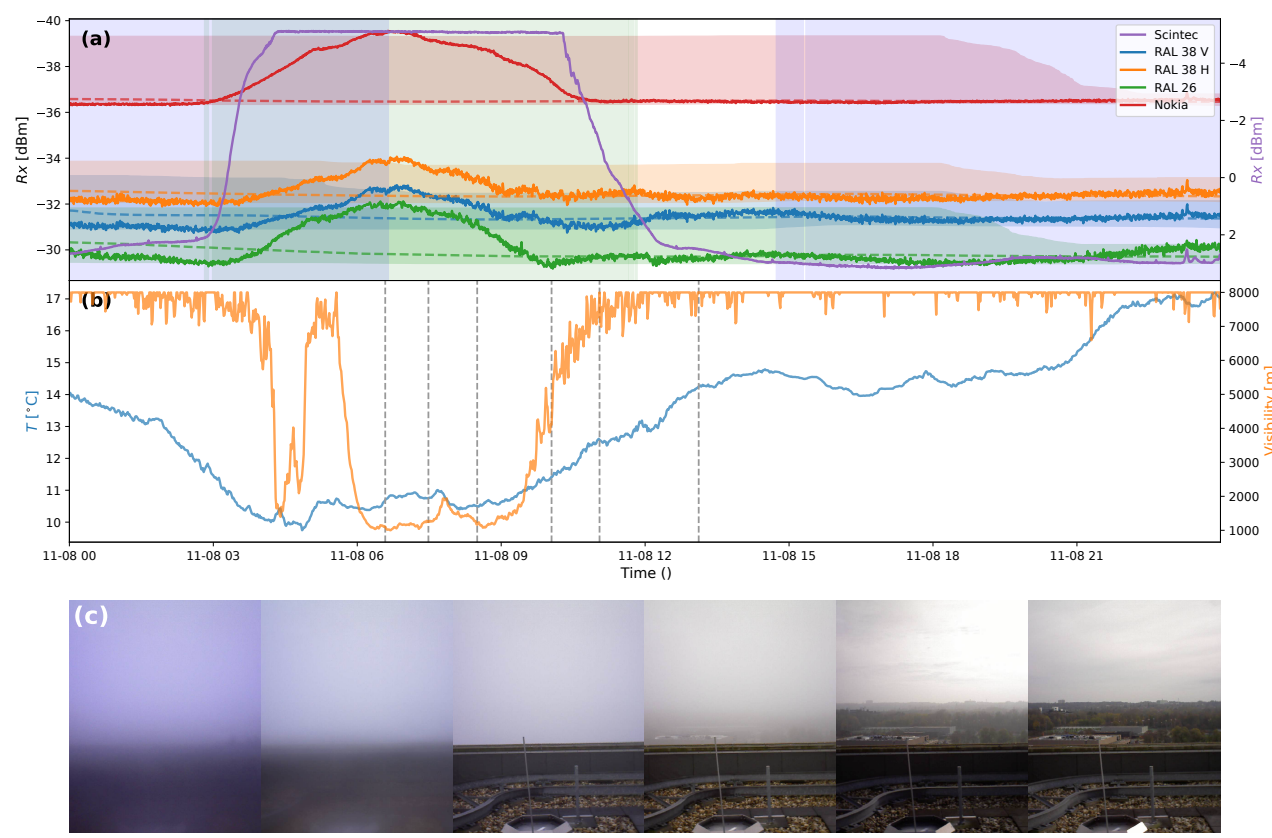

Figure 2.12: Time series of an event on 8 November 2015. (a) Received power levels at the detectors (solid lines) and reference levels (median over dry periods in a $24 \mathrm{~h}$ moving window: dashed lines). Periods with a negative net radiation flux at the surface are indicated with blue shading. Periods with a relative humidity $>90 \%$ are indicated with green shading. (b) Several atmospheric variables measured at the "Veenkampen" meteorological station: visibility and ambient air temperature at $2 m$ indicated with orange and blue lines, respectively. (c) Images from the time-lapse camera at the location of the receiving antennas aimed along the link path. The times at which these images were captured are indicated by the vertical dotted lines.

as the principal cause, from correlations alone. If we take the visibility as indicative of the amount of fog, then we can see that they indeed occur often (but not always) at the same time.

In the case shown in Fig. 2.12, a different fog event is shown in more detail. In this case, time-lapse camera footage was available for a significant portion of the event, and is shown in Fig. 2.12c. Here again, strong attenuation is experienced by all the links with a peak attenuation of $3 \mathrm{~dB}$ in the case of the Nokia link, yet none of the disdrometers detect precipitation. Therefore, it is likely that we are dealing with a different attenuating phenomenon than precipitation. Using the basic rainfall retrieval algorithm, this event would result in an accumulated rainfall depth of $26 \mathrm{~mm}$. As the time series of attenuation is smoother than we would expect of rainfall, we are likely dealing with antenna wetting due to either dew formation, or the result of fog. The effect of fog on microwave link attenuation has also been observed by, Liebe et al. (e.g. 1989); David et al. (e.g. 2013). How- 
ever, as mentioned in those studies, the theoretical attenuation by fog droplets themselves is only $0.75 \mathrm{~dB}$ at $38 \mathrm{CHz}$ for a $1 \mathrm{~km}$ path and a very high liquid water content of $0.8 \mathrm{~g} \mathrm{~m}^{-3}$. Therefore, no more than $1.5 \mathrm{~dB}$ attenuation can be expected due to the fog itself and an attribution to fog must also include the wetting of the antennas. Observation of the accompanying time-lapse camera footage (Fig. 2.12c) reveals a heavy fog during the early morning, which gradually clears up concurrently with the decrease in attenuation in the late morning. No camera footage was available at night during the increasing leg of the attenuation signal, because the cameras cannot record during low-light. However, comparison of the visibility data from the nearby weather station (Fig. 2.12b) with the pattern of attenuation, seems to undermine a direct relationship with fog. Visibility measurements from the NIR link along the path itself are of limited use in this case, as the fog is so heavy that the attenuation is "saturated" for most of the duration. The striking correspondence of the sign switch of both net radiation and attenuation increase makes dew formation the most likely interpretation.

\subsubsection{Wet antennas}

Near the start of the measurement period a simple test case was performed to assess the effect of wet antennas on rainfall retrieval. During a dry sunny day (12 September 2014), while the ambient temperature was $21^{\circ} \mathrm{C}$, both the Nokia and the $\mathrm{RAL} 38 \mathrm{CHz}$ receiving antennas were artificially wetted in short bursts using a spray bottle. The antennas were wetted until visually saturated and then allowed to dry in the sun. In this way, the attenuating effect of wet antennas can be observed, decoupled from the attenuating effect of raindrops in air. The RAL $26 \mathrm{CHz}$ link was not included in the test, as it was not yet installed at the time; however, the antenna cover design and material is identical to that of the RAL $38 \mathrm{CHz}$ link (aside from its diameter) and thus it is assumed that the effect is similar.

In Fig. 2.13a the resulting attenuation signal is shown. It is seen that wetting of one antenna of the Nokia link system can result in an extra attenuation of 3 to $5 \mathrm{~dB}$, which is of the same order of magnitude as what is observed in dew and fog events (where presumably both antennas of a link are affected). This corresponds with a rain intensity of 15 to $22 \mathrm{~mm} \mathrm{~h}^{-1}$ using the power law derived in Sect. 2.4.1.3 (shown in Fig. 2.13b). The signal then follows an exponential decay pattern due to drying, where the time for the attenuation to decrease by $95 \%$ is $3 \mathrm{~min}$. The RAL link response to wetting is completely different, which may be related to the way water collects on the antenna cover surface. The extra attenuation due to wetting is only 1 to $3 \mathrm{~dB}$ and the decay has two distinct stages. The initial peaks drop in less than a second after the spray stops, with no discernible decay pattern. This drop can range from 0.3 to $1.3 \mathrm{~dB}$. However, after the initial peak, the attenuation does not drop to the baseline level; it stays at relatively constant elevated level after the spray. After each new spray the level may or may not change; not necessarily to a higher level. Only 21 min after the last spray, which was administered shortly before 14:56, has the attenuation fully decayed to the dry level (the full length of the decay is not shown on the graph). While the observations of the Nokia link conform to the empirical model of Minda and Nakamura (2005), which effectively describes the drying of a thin water film 


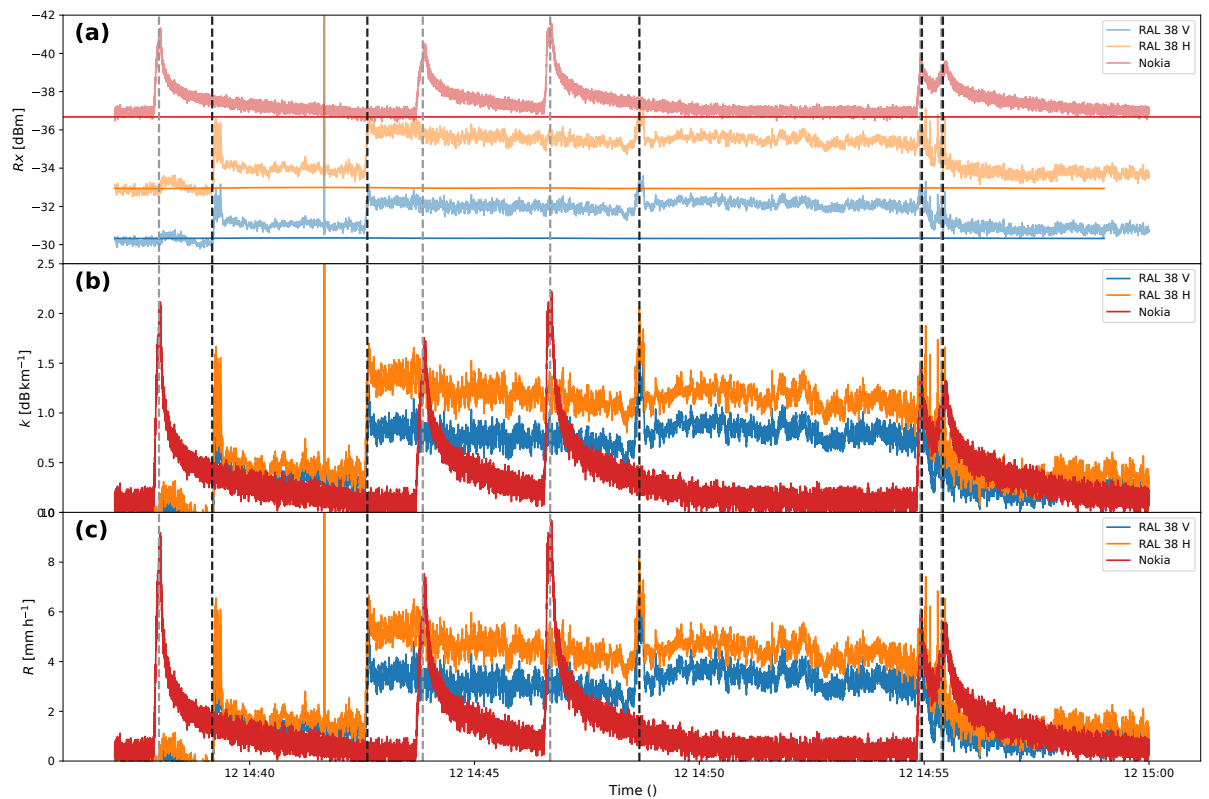

Figure 2.13: Time series of the wet antenna experiment on 12 September 2014. (a) Received power levels at the detectors, with the reference levels indicated in darker hues. The reference levels are singular values manually fitted for this event. These are the raw $20 \mathrm{~Hz}$ sampled data, not the $30 \mathrm{~s}$ resampled data. The dotted vertical lines indicate the moments when a water spray was applied, with the dark grey lines indicating sprays on the RAL antenna and the light grey lines indicating sprays on the Nokia antenna. (b) Specific attenuation of the links. (c) Derived rainfall intensities using the $R-k$ power law.

on the antenna, the observations of the RAL link do not. Figure 2.14, containing photos of the antennas just after wetting, shows that while a thin nearly uniform film of water has formed on the Nokia link antenna, this does not hold for the RAL link antenna cover. Instead of forming a smooth layer, the hydrophobic material of the antenna cover forces the water to either run off immediately or collect into a few large beads. The runoff leads to a reduced peak attenuation and an immediate drop afterwards, as the surface is never fully covered with water. However, the bead formation leads to a long secondary decay time, as the reduced surface to volume ratio (as compared to a uniform layer) hampers evaporation. As recorded video footage shows, with each new squirt of water, some new beads form while some others grow and fall off. The number and sizes of the beads remaining afterwards is highly variable, which might give a tentative explanation as to why the secondary attenuation level changes after each burst (and can even become lower than the previous level). It is interesting to note that Schleiss et al. (2013) also report individual drops on the antennae, rather than a sheet of water. They find drying times ranging from 0.5 to $5 \mathrm{~h}$, which is considerably longer than the under $3 \mathrm{~min}$ reported by Leijnse et al. (2008) and Minda and Nakamura (2005). The results of this experiment seem to indicate 

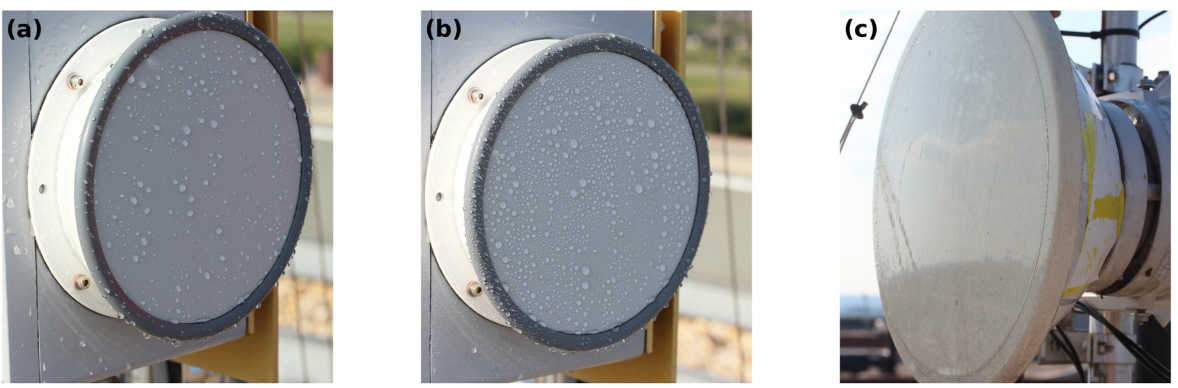

Figure 2.14: Photographs of the receiving antenna covers, during the wet antenna experiment, taken just after the antennas were sprayed. Panels (a) and (b) are two instances of the RAL $38 \mathrm{CHz}$ cover. Panel (c) is the Nokia Flexihopper cover.
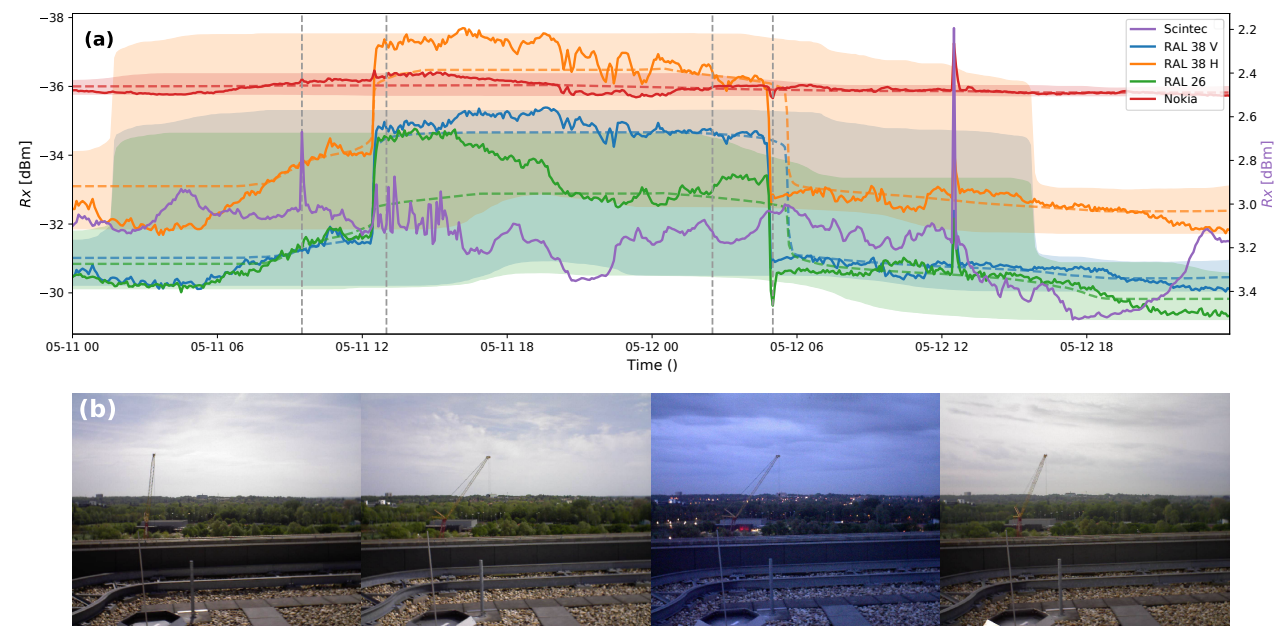

Figure 2.15: Time series of an event on 11 May 2015. (a) Received power levels at the detectors as solid lines, with the reference levels (median over dry periods in a $24 \mathrm{~h}$ moving window) indicated by dashed lines. The 5th and 95 th percentile power levels overdry periods in a $24 \mathrm{~h}$ moving window are indicated by the coloured shading. (b) Images from the time-lapse camera at the location of the receiving antennas aimed along the link path. The times at which these images were captured is indicated by the vertical dotted lines.

that bead formation versus sheet formation is a major reason for the disparity in drying times.

\subsubsection{Clutter}

Figure 2.15 shows an example of a remarkable event that occurred several times in the observation period. Figure 2.12a displays the received power for the four microwave link 
signals in the period of 10 to 12 May 2015. There is a sudden sharp signal decrease and $18 \mathrm{~h}$ later a subsequent increase towards normal levels. The disdrometers do not indicate any significant rainfall event during this time. Inspection of the time-lapse camera footage (shown in Fig. 2.15b) indicates that a large metal construction crane was positioned exactly in front of the link path during this time about $200 \mathrm{~m}$ from the receivers, while it is positioned differently and regularly moving outside this time period. A few hours later we see another momentary drop in the received signal levels at which point the crane moves swiftly through the path. The Nokia link detects no signal loss during the long period, but it does on other similar occasions. As the radius of the first Fresnel zone at this distance is only $1.3 \mathrm{~m}$ and the centres of the Nokia antennas are about $0.5 \mathrm{~m}$ higher than the centres of the RAL antennas, there is a distinct possibility that in some instances the obstacle was only blocking some of the links.

We see similar patterns on multiple occasions and each time a large metallic object was positioned in the path. On other occasions, for example, a window cleaners' metal gondola crane was the cause of the attenuation. These kinds of temporary obstructions of the link path cannot be ruled out in operational settings, and most of the time no continuous visual observations are available. Therefore, it would be advantageous to be able to recognize these signal patterns and remove them algorithmically.

\subsubsection{Compound phenomena}

There are several anomalies present in the dataset that cannot be easily explained by any single observed atmospheric phenomenon as described in the previous sections.

Figure 2.16 shows another example of a rain event. It can be seen that the link attenuation signals mimic the temporal dynamics in the rainfall. It can also be seen that the RAL $38 \mathrm{CHz}$ link shows continued attenuation after the first rain event has stopped. One possible explanation could be because the antennas become wet themselves, which contributes extra to attenuation. However, the duration of the effect in this instance is almost $3 \mathrm{~h}$, which is somewhat inconsistent with the results from Sect. 2.4.6, which suggests a duration in the order of $21 \mathrm{~min}$. It is also inconsistent with some other events during this experiment when only a short attenuation period was observed after a precipitation event. Here as well, after the second rain shower, no lingering attenuation is observed. However, it is consistent with the findings of Schleiss et al. (2013). The Nokia link shows no lingering attenuation in both cases, which is consistent with the results from the wet antenna experiment. It is hard to specify why lingering attenuation effects occur after some rain events and not after others, but ambient conditions such as air humidity, temperature and wind speed might play a role here. The relative humidity hovers around $90 \%$ after the first event, while it drops to $80 \%$ directly after the second event. Concurrently, temperature increases from 14.5 to $18^{\circ} \mathrm{C}$ and wind speed increases from 1 to $6 \mathrm{~m} \mathrm{~s}^{-1}$. It is even harder to specify why some antennas are much more affected by the phenomenon than others at a given time; however, the beading effect of the wet antenna with hydrophobic antenna cover might be related to this. 


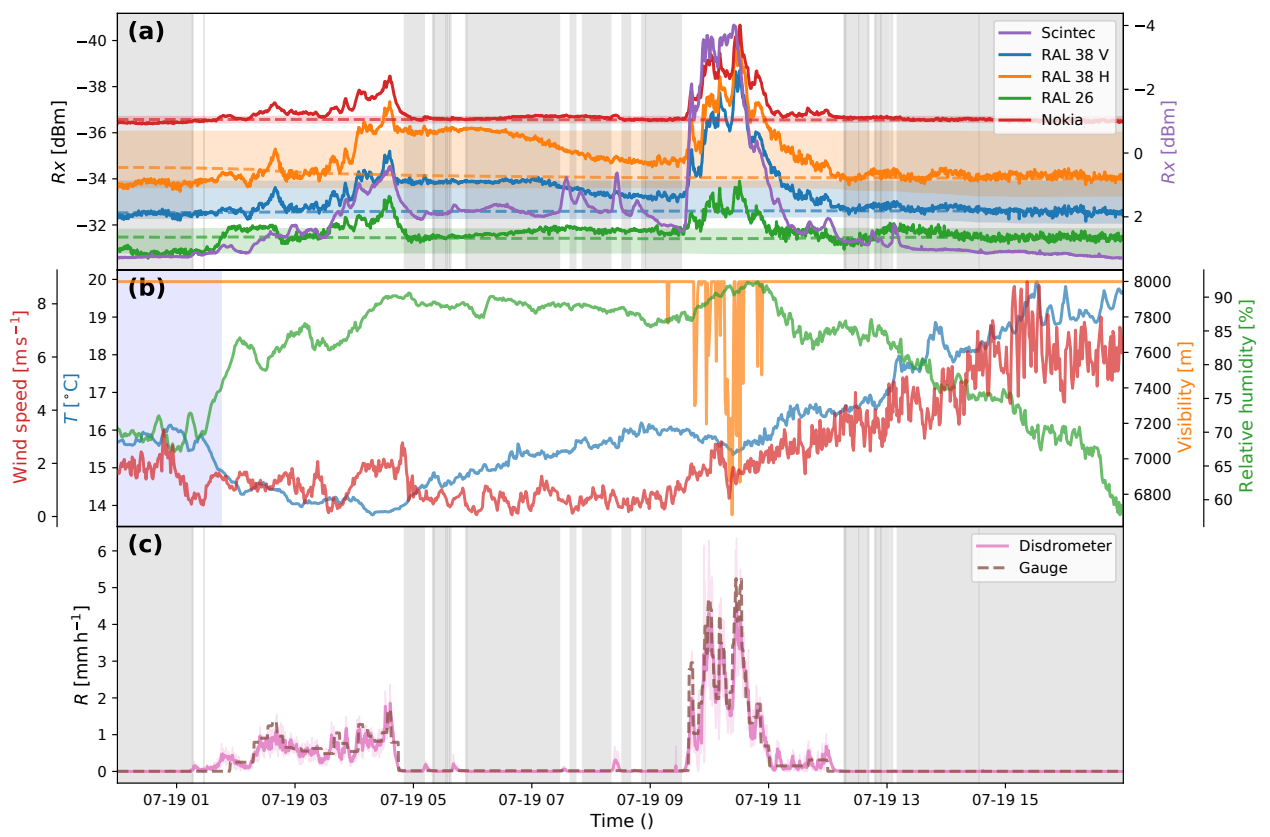

Figure 2.16: Time series of an event on 19]uly 2015. (a) Received power levels at the detectors (solid lines) and reference levels (median over dry periods in a $24 \mathrm{~h}$ moving window: dashed lines). The 5 th and 95 th percentile power levels over dry periods in a $24 \mathrm{~h}$ moving window are indicated by the coloured shading. (b) Several atmospheric variables measured at the "Veenkampen" meteorological station: relative humidity, visibility, and ambient air temperature at $2 \mathrm{~m}$, and wind speed indicated with blue, orange, green, and red lines, respectively. Periods with a negative net radiation flux at the surface are indicated with blue shading. (c) The spatial weighted average rainfall intensities derived from the disdrometers are indicated by the pink line, with the weighted standard deviation among the disdrometers displayed by the pink shaded area. The rainfall intensities derived from the tipping bucket gauge are indicated with the dashed line. Dryperiods, as indicated by the disdrometers, are displayed by grey shaded areas. 


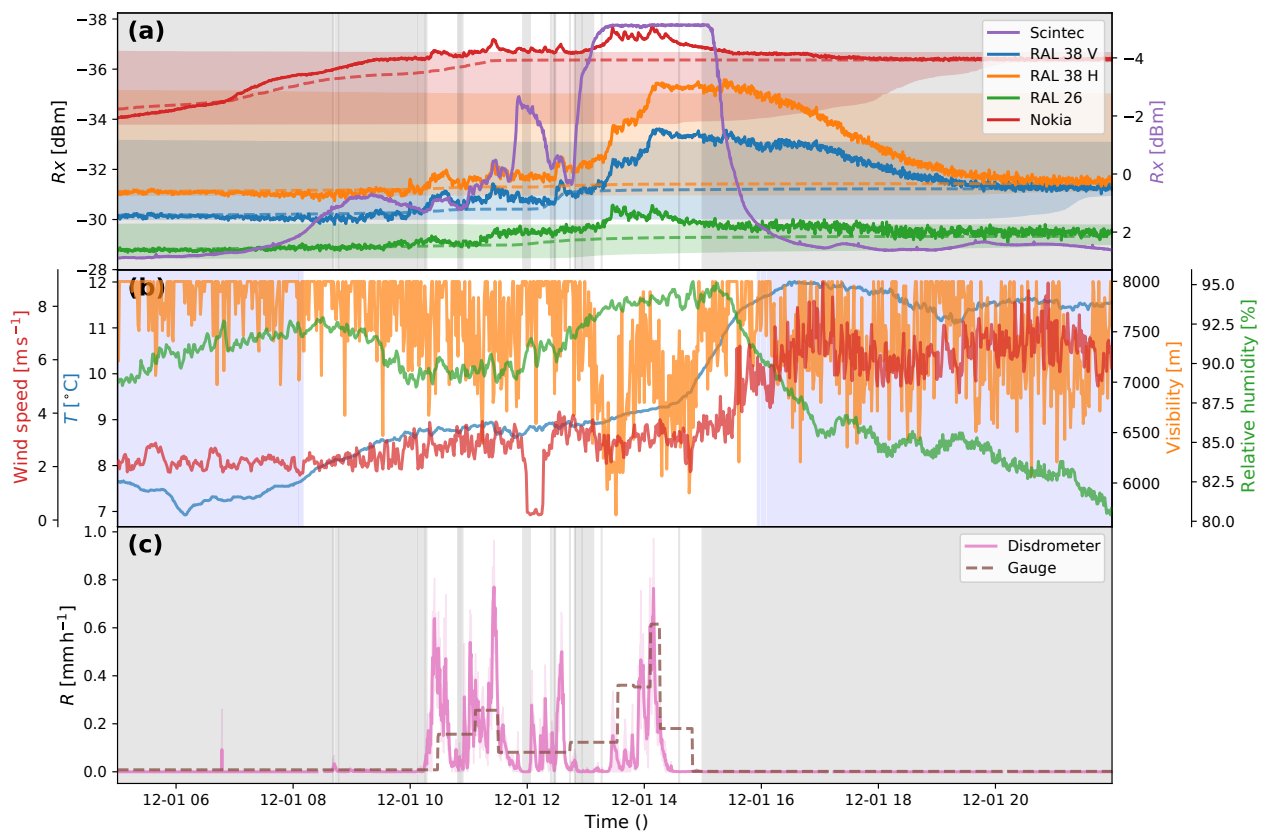

Figure 2.17: Time series of an event on 1 December 2015. (a) Received power levels at the detectors (solid lines) and reference levels (median over dry periods in a $24 \mathrm{~h}$ moving window: dashed lines). The 5th and 95th percentile of power levels over dry periods in a $24 \mathrm{~h}$ moving window are indicated by the coloured shading. (b) Several atmospheric variables measured at the "Veenkampen" meteorologicalstation: relative humidity, visibility, and ambient air temperature at $2 \mathrm{~m}$, and wind speed indicated with blue, orange, green, and red lines, respectively. Periods with a negative net radiation flux at the surface are indicated with blue shading. (c) The spatial weighted average rainfall intensities derived from the disdrometers are indicated by the pink line, with the weighted standard deviation among the disdrometers indicated with the pink shaded area. The rainfall intensities derived from the tipping bucket gauge are indicated with the dashed line. Dry periods, as determined with the disdrometers, are represented by grey shaded areas. 
The examples given above have been simple cases where rain and other attenuating phenomena occur in an isolated fashion. These cases are important to be able to investigate and explain these phenomena. However, many times throughout the investigated period multiple phenomena have occurred simultaneously, which is a complicating factor for retrieval algorithms. Figure 2.17 provides an example of a complex event occurring on 1 December 2015. In this case, there is a simultaneous light drizzle and fog. Figure $2.17 \mathrm{c}$ shows that the disdrometers register rain intensities of below $1 \mathrm{~mm} \mathrm{~h}^{-1}$ over a period of over $4 \mathrm{~h}$. Despite the low intensity, the drizzle does produce attenuation of the links between 10:00 and 13:00, as can be seen in Fig. 2.17a. Fog rolls in at around 13:00 as evidenced by the time-lapse footage (not shown here) and substantiated by the increasing relative humidity and decreasing visibility as seen in Fig. 2.17b. From 13:00 till roughly $14: 30$ fog and drizzle occur simultaneously and both contribute to the attenuation. At 15:30 the fog has blown over or has dissipated. This is captured well by the Nokia link attenuation signal. The RAL link signals remain attenuated until 20:00. This could be due to the antenna covers still being wet. As was pointed out in Sect. 2.5.6, Due to bead formation, the hydrophobic antenna covers can stay wet much longer. Indeed, from 16:00 onwards, net radiation flux towards the surface is negative (indicated by the shading in Fig. 2.17b) and thus only wind drying can take place. The simultaneous occurrence of drizzle and fog could pose a problem for binary dew filtering algorithms such as the one proposed by Overeem et al. (2016b).

\subsection{Conclusions}

In this chapter we have tested a straightforward rainfall retrieval algorithm applied to the microwave link measurements on the basis of a power-law relationship and compared the results with five disdrometers positioned along the path. This allows us to assess what the quality of a retrieval would be without taking into account the effect of other sources of attenuation. It is seen that there is a strong overestimation of rainfall intensities by the microwave links when compared to the disdrometers, when no corrections for the phenomena discussed in this chapter are applied. The response of the link signals to liquid precipitation in terms of additive and multiplicative bias is of the same order of magnitude in both drizzle and heavier rainfall. This means that drizzle is much harder to quantify than heavier rainfall events because there is an additive bias of roughly $0.6 \mathrm{~mm} \mathrm{~h}^{-1}$ in the Nokia link and roughly $2 \mathrm{mmh}^{-1}$ in the RAL links, i.e. of the same order of magnitude as typical rainfall intensities in drizzle.

There are significant differences in the accuracy of the rainfall retrieval between the two different makes of microwave links that we used, operating at the same frequency and polarization. In general, the commercial link has a less noisy and more unambiguously interpretable signal response than the dedicated research link. The latter overestimates the rainfall intensity more during pure rainfall events.

Unfortunately, there were only a few minor instances of snow and ice pellets occurring in the experimental area during the measurement period. Due to the limited amount 
of data no meaningful empirical conclusions can be drawn concerning the accuracy with which solid precipitation can be retrieved.

Of the three microwave links that we tested, two exhibited a significant dependence of received signal level on the ambient temperature. The received signal level of the research links was attenuated by -0.1 to $-0.2 \mathrm{~dB} \mathrm{~K}^{-1}$. The commercial link received signal level was not strongly attenuated by temperature, although a temperature dependence was found for a period where little other dynamic attenuating phenomena (rain, dew, etc.) were present. In this case, the (apparent) attenuation due to temperature was only $-0.024 \mathrm{~dB} \mathrm{~K}^{-1}$. Whether temperature dependence is a problem for rainfall retrieval that needs to be corrected for is thus dependent on the specific link hardware and not on the frequency ( 26 or $38 \mathrm{GHz}$ ) or polarization of operation.

We find attenuation due to fog or dew for all three microwave links. The peak magnitude of this attenuation in a typical event is 2 to $3 \mathrm{~dB}$. The attenuation in the commercial link is slightly stronger than in the research links. The peak attenuation caused by fog or dew is of the same order of magnitude as a moderate rainfall event. If one would interpret such an event as rain the accumulated rainfall depth would be in the order of tens of millimeters. Therefore, it is important to correct for this phenomenon to obtain accurate rainfall data.

The use of a hydrophobic antenna cover should in principle reduce overestimation due to wet antennas. However, in practice, it also leads to bead formation which has adverse consequences. The beads take much longer to evaporate than a thin layer of water under similar circumstances, so after rainfall has stopped, or after dew conditions have subsided, the attenuation lingers much longer. More importantly, they make the magnitude of the attenuation during this drying-up period less predictable, because the configuration of the beads on the antennas is unpredictable. As such, we would tentatively recommend against the use of hydrophobic antenna covers for research links, although a more robust experiment might be needed to confirm this conclusion.

We also observed that in the typical urban environment temporary obstructions of the link path can lead to a link attenuation of several $d B$. Future research should be conducted to detect such sudden shifts in the baseline level and automatically correct for this.

Finally, we found that several of these phenomena can contribute to the apparent attenuation of the link signal concurrently. In particular, we show that light drizzle and fog can appear at the same time, which would lead to an overestimation of the rainfall intensity. Many current microwave link retrieval implementations currently rely on an a priori classification of a given time interval as either a rainy or dry period and assume the baseline signal level to be constant during a rainfall event. This is not sufficient for intervals where multiple effects play a role. Therefore, we believe the development of filtering methods which do not rely on an explicit binary classification should be explored.

In general, the use of two different microwave links operating at the same frequency 
along the same path during the same time (which should theoretically produce the same results) resulted in two remarkably different signal responses to rainfall and other attenuating phenomena. Therefore, we recommend that, when making use of data from commercial networks, note should be taken of the specific manufacturers and models the network is comprised of and the retrieval algorithm should be optimized for those link devices (i.e. by putting an example device in a test environment). This is especially relevant when parts of the network are supplied by different manufacturers. However, the remarkable stability of the Nokia link does demonstrate the value of commercially available microwave links for precise rainfall measurements when sampled at high frequencies.

We have demonstrated the effect of several complicating phenomena in typical microwave attenuation data for rainfall retrieval. The collected data from this experiment could be used to assess the effect of different sampling strategies used by commercial microwave links from cellular communication networks in operational settings. Moreover, the experimental data can also be used as a test dataset to improve existing algorithms (e.g. Overeem et al., 2016a) and devise corrections for the plethora of attenuating phenomena described in this chapter. 


\title{
Chapter 3
}

\section{Rainfall spatio-temporal correlation and intermittency structure}

\begin{abstract}
We investigate the spatio-temporal structure of rainfall at spatial scales from $7 \mathrm{~m}$ to over $200 \mathrm{~km}$. We used data from two networks of laser disdrometers with complementary interstation distances in two Dutch cities and a Dutch nationwide network of automatic rain gauges. The smallest aggregation interval collected by the disdrometers was $30 \mathrm{~s}$, while the automatic rain gauges provided 10 minute rainfall sums. This study aims to supplement other micro- $\gamma$ investigations (usually performed in the context of variability within a weather radar pixel) with new data, while characterizing the correlation structure over an extended range of scales. To quantify the variability, we employ a modifiedexponential model fitted to the spatial correlograms and characterize the parameters of the model as functions of the temporal aggregation interval. This common method allows for meaningful comparison with other studies. We also separately analyzed the intermittency of the rainfall observations. We show that a single parameterization can coherently describe the variability across a wide range of scales. Limiting the range of scales to those typically found in micro- $\gamma$ variability studies skews the parameterization and reduces its applicability to larger scales.
\end{abstract}

This chapter is based on: Van Leth, T.C., Overeem, A., Leijnse, H. and Uijlenhoet, R., Rainfall spatio-temporal correlation and intermittency structure from micro- $\gamma$ to meso- $\beta$ scale, to be submitted. 


\subsection{Introduction}

Quantification of small-scale rainfall variability is important for the design and operation of small-scale sensor networks for flood prediction, particularly in urban areas. It is also important for the assessment of the spatial representativeness of path or area averaged measurement methods such as microwave links, radar and satellite remote sensing. Furthermore the spatial and temporal patterns of variability provide insight into the physical structure of rain and can be used for the construction of rain simulations.

Several measurement campaigns have been carried out in the past decades in order to assess the spatio-temporal variability of rain in a statistical sense at scales comparable to a typical weather radar pixel, corresponding to the micro- $\alpha$ to micro- $\gamma$ scales in the framework of Orlanski (1975). A network of laser disdrometers has been used before to measure the temporal variability of drop size distributions by Tapiador et al. (2010) and Jaffrain and Berne (2012). Jameson et al. (2015) used a similar disdrometer network with logarithmic spacing in order to investigate clustering in rainfall fields. Habib and Krajweski (2002), Ciach and Krajewski (2006), Villariniet al. (2008) and Peleg et al. (2013) did a similar analysis using rain gauges at small scale. Meanwhile, at the meso-scale Van de Beeket al. (2011) used a Dutch nationwide automatic rain gauge network to analyse rain variability at the larger spatial scales at an hourly time scale and intergauge distances of $30-300 \mathrm{~km}$. Schuurmans et al. (2007) employed the same dataset in conjunction with a micro-scale tipping bucket gauge network and radar for high-resolution rainfall field prediction.

Although quite some empirical data is therefore available to investigate spatial variability on scales smaller than $1 \mathrm{~km}$ with sub-hourly aggregation intervals, there remains much uncertainty regarding the applicability of these measurements. Different measurement campaigns find different parameterizations and not enough independent measurement campaigns have been carried out to determine whether these differences are related to climatology, instrumentation, the different ranges of separation distances or other causes. Therefore, more independent spatial variability experiments are needed as well as an effort to reconcile the different distance ranges. This chapter aims to contribute towards filling that gap using data that was originally collected to validate rainfall measurements using microwave links with separation distances ranging from $9 \mathrm{~m}$ to $2200 \mathrm{~m}$. We supplement this data with a medium range dataset collected in the city of Rotterdam as part of an operational hydrometeorological network. Finally, we also use new data from the Dutch nationwide gauge network which is now available at 10 min aggregation intervals. We aim to test the consistency of the findings at different scales and as part of different measurement campaigns.

\subsection{Data}

\subsubsection{Datasets}

We have used disdrometer data from a field campaign (see Chapter 2) conducted in the Dutch town of Wageningen. This field campaign was set up to provide a test bed for mi- 
crowave link rainfall retrieval algorithms. The five OTT Parsivel disdrometers were situated roughly on a line of $2.2 \mathrm{~km}$ length. see Fig. 3.1a. Two of the disdrometers were situated on top of the same building only $7 \mathrm{~m}$ apart, the other three were placed on other buildings in the Wageningen urban area. The disdrometers provided an integrated drop size distribution per 30 seconds and data from all the disdrometers was available from March 2015 until December 2015. From these drop size distributions, rainfall intensities where calculated (see Van Leth et al. (2018) for details). These particular disdrometers were used before in Lausanne, Switzerland (Jaffrain et al., 2011) where they had been part of the disdrometer network used by Jaffrain and Berne (2012).

Another dataset is used in order to extend the analysis to larger spatial and temporal scales. This dataset was collected in and around the Dutch city of Rotterdam, around $50 \mathrm{~km}$ from Wageningen, in the period of January 2010 till October 2011. It was collected by the municipality of Rotterdam using six Thiess LPM laser disdrometers. These disdrometers are functionally quite similar to the OTT Parsivel disdrometers used in Wageningen. The locations of these measurement stations are shown in Fig. 3.1b. These disdrometers provided rain intensity estimates over a 1 minute aggregation interval; We did not have the actual drop size distributions available. This dataset is used to provide spatial correlation estimates across distances ranging from $3 \mathrm{~km}$ to $30 \mathrm{~km}$.

To extend the range of spatial scales further we also make use of data from a Dutch nationwide automatic rain gauge network operated by the Royal Netherlands Meteorological Institute (KNMI). This provides rainfall depths aggregated to 10 minutes at 31 locations throughout The Netherlands (Fig. 3.1c). In contrast to the tipping bucket gauges used in other studies, these gauges measure the rainfall depth using the displacement of a float placed in a reservoir and can therefore record continuously. This validated dataset includes the same measurement period as that over which the Rotterdam dataset was measured. The range of interstation distances $d$ overlaps somewhat with the Rotterdam dataset (20 km $<d<315 \mathrm{~km}$ ). The network of automatic rain gauges is the same as was used by Van de Beeket al. (2011). However, they used a different measurement period (1979-2009) and they only had access to hourly rainfall sums.

\subsubsection{Quality control}

The Wageningen disdrometer dataset included an indication of precipitation type. We used the product of this build-in Parsivel algorithm to exclude anything other than liquid precipitation. We also set any intervals where the Parsivel algorithm indicates dry weather to $0 \mathrm{~mm}$.

The Rotterdam dataset did require some quality control before it could be reliably compared to the Wageningen dataset. Because little metadata was available from the disdrometers, we used gauge-adjusted radar pixels containing the disdrometer locations as a reference to eliminate faulty measurements. This weather radar data was obtained using the two C-band radars operated by KNMI and corrected using both the automatic 

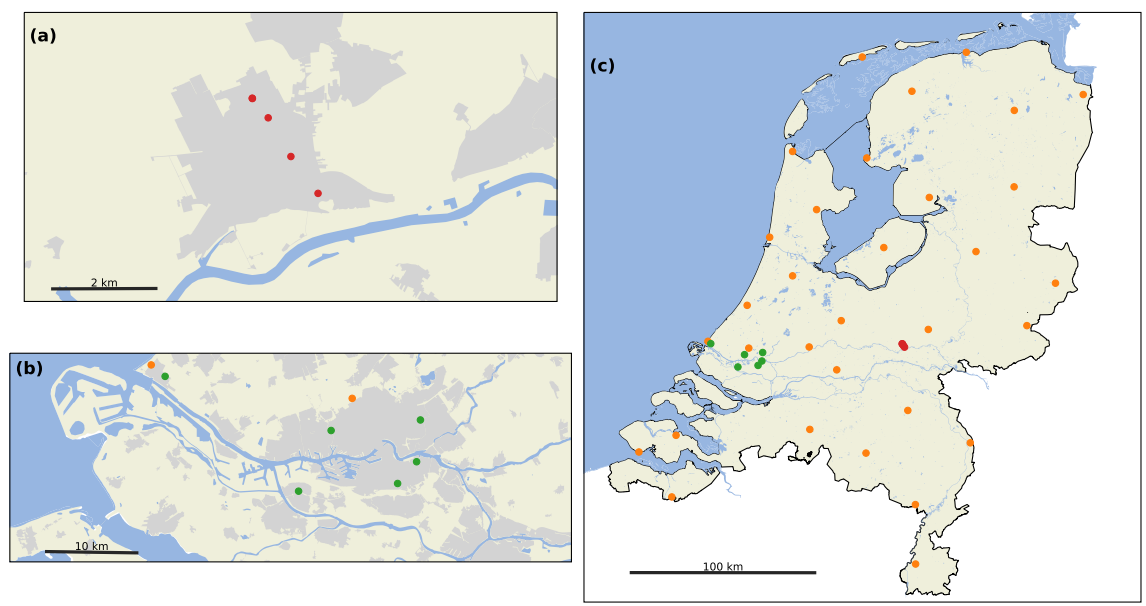

Figure 3.1: a) Relative locations of the disdrometers in Wageningen. b) Relative locations of the disdrometers in Rotterdam. c) Relative locations of the automatic rain gauges and disdrometers in The Netherlands.

rain gauge network and a network of 325 manual gauges (Overeem et al., 2009a, b, 2011) ${ }^{1}$. The temporal resolution is 5 minutes. Of course, weather radar and ground based measurements cannot be directly compared even when the magnitude is adjusted. Because the situation at $1.5 \mathrm{~km}$ height can be different than at ground level, because the radar pixel represents a volumetric average as opposed to a point measurement and because the centers of the pixels do not align perfectly with the position of the disdrometers, directly comparing the two measurements would yield many false positives for exclusion. We recognize several aspects of the data that could lead to intervals being erroneously categorized as faulty measurements: Intermittency of precipitation within an event; a lag or lead time for an entire event; and small-scale spatial variability in magnitude which is not captured by radar. Since spatio-temporal variability at the small scale is exactly what we are investigating here, it is of the utmost importance that we do not unduly influence our results by erroneously excluding events that simply exhibit a high variability. In order to do so we have devised the following filtering algorithm: We first apply a 3-hour moving average filter to both the disdrometer timeseries (after first aggregating to 5 minutes) and the radar timeseries. This smooths out small-scale intermittency. Next, we compare the smoothed radar timeseries and the smoothed disdrometer timeseries and evaluate the following equation:

$$
\left(R_{\text {disdro }, 3 h} / R_{\text {radar }, 3 h}>a\right) \wedge\left(\left|R_{\text {disdro }, 3 h}-R_{\text {radar }, 3 h}\right|>b\right)
$$

If the above equation evaluates as true for a given 5 minute interval then the interval is excluded. Here $a=2$ and $b=1 \mathrm{~mm} \mathrm{~h}^{-1}$, so that intervals are only excluded when the

\footnotetext{
${ }^{1}$ freely available at https://data.knmi.nl/datasets/rad_nl25_rac_mfbs_5min/2.0.
} 
3-hour moving averages diverge substantially. this prevents us from excluding intervals displaying small scale-spatial variability.

\subsection{Methodology}

As an estimator for the spatial correlation we use Pearson's correlation coefficient $\rho$.

$$
\rho=\frac{\overline{X Y}-\bar{X} \bar{Y}}{\sqrt{\left(\overline{X^{2}}-\bar{X}^{2}\right)\left(\overline{Y^{2}}-\bar{Y}^{2}\right)}}
$$

where $X$ and $Y$ are the two stations to be compared and the means are taken over the entire time-series. This in then applied for every combination of two stations in a given dataset. We use a bootstrap procedure to estimate the uncertainty in the correlation coefficient. Here we randomly take $N$ intervals from the $N$-length timeseries and recalculate the correlation coefficient. We perform this procedure 100 times for every combination of stations and calculate the standard deviation from the resulting distributions. This we take as a measure of confidence in the correlation coefficients.

We determine the decorrelation distance with the following 2-parameter exponential spatial correlation model:

$$
\rho(d)=e^{-\left(\frac{d}{d_{0}}\right)^{s_{0}}}
$$

where $d$ is the distance, $d_{0}$ is the decorrelation distance and $s_{0}$ is the shape parameter. This is similar to the 3-parameter exponential model suggested by Krajewski et al. (2003), except we omit the nugget parameter. We take the decorrelation distance to be a reliable measure of the spatial variability for a given temporal accumulation interval. In doing so we therefore assume that the rainfall variability is isotropic (i.e. not dependent on direction). To fit the optimal parameters $d_{0}$ and $s_{0}$ we use a standard LevenbergMarquardt non-linear least squares implementation, where we have used the uncertainties calculated with the bootstrap procedure as weights in the cost function. The confidence bands for the non-linear regression curves are determined using a Monte-Carlo technique. Here, the numerically estimated parameter covariances are used to generate 100 parameter instances of a multivariate Caussian distribution. Using Eq. (3.3), we get 100 instances of the correlation coefficient at each distance, and we determine the $5^{\text {th }}$ and $95^{\text {th }}$ percentile of the correlation coefficient for each distance. We have performed the above-mentioned procedure for several temporal aggregation periods ranging from $30 \mathrm{~s}$ (depending on the available data) to $48 \mathrm{~h}$.

We also investigate the spatial intermittency of the rainfall. That is, disregarding the quantity of the rainfall, what is the spatial variability in dry and rainy sub-intervals within a rainy period. As a measure of spatial intermittency we calculate the following quantity:

$$
I_{i j}=\frac{N_{\left(R_{i, t}>0 \wedge R_{j, t}>0\right)}}{N_{\left(R_{i, t}>0 \vee R_{j, t}>0\right)}}
$$


Here $N_{\left(R_{i, t}>0 \wedge R_{j, t}>0\right)}$ is the number of intervals where both stations register rain throughout the aggregation interval, while $N_{\left(R_{i, t}>0 \vee R_{j, t}>0\right)}$ is the number of intervals where at least one of the stations registers rain at least once during the aggregation interval. By registering rain, we mean here that the rainfall intensity at the original (smallest) aggregation level is nonzero. We will refer to $I$ as the 'intermittency ratio'.

\subsection{Results}

\subsubsection{Spatial correlation}

The correlations between the rainfall intensities from two stations are plotted against the distances between them. This is done for both the Wageningen and the Rotterdam disdrometer datasets as well as the nationwide gauge dataset. The decorrelation distances are determined using Eq. (3.3) for different resampled aggregation intervals. The results are shown in Fig. 3.2. The optimal values found for the fit parameters are given within the figure together with the standard deviations estimated in the regression procedure.

It can be seen that rainfall estimates from the two closest stations ( $7 \mathrm{~m}$ apart) are already highly correlated at a 30 second integration period $(\rho=0.966 \pm 0.002)$. At a 1 minute integration period and a distance of roughly $500 \mathrm{~m}$ ( $483 \mathrm{~m}$ and $490 \mathrm{~m}$ ) the correlation coefficients are $0.860 \pm 0.008$ and $0.875 \pm 0.008$, respectively. This is clearly higher than what Villarini et al. (2008) found for their smallest intergauge distance bin of roughly 500 $\mathrm{m}$ at a 1 minute integration interval. They found correlation coefficients ranging from 0.21 to 0.38 for the Brue catchment using tipping bucket rain gauges. Tapiadoret al. (2010) only analysed a few single days, but for this aggregation period and interstation distance have found similarly high correlation coefficients (but for the radar reflectivity), ranging between 0.90 and 0.97 depending on the date. They used instruments of the same type used in this experiment. Including an additional nugget parameter in the decorrelation model does not improve the goodness-of-fit for either of the two datasets. The coefficient of determination, $R^{2}=0.99$ for the $30 \mathrm{~s}$ aggregation level for the Wageningen dataset, and $R^{2}$ is found to be 0.95 for the 1 min Rotterdam dataset, with no significant difference using either model.

The fitted curves based on the Wageningen dataset and the Rotterdam dataset are quite dissimilar and gradually become more so as the aggregation period increases. However, it also clear that a direct comparison between the two is impossible given their respective interstation distance ranges do not overlap. Especially with regards to the Wageningen dataset it should be noted that the optimally fitted decorrelation distances are in all cases outside of the range of actually measured distances. Therefore, the range of interstation distances in the Wageningen dataset is insufficient to determine the actual decorrelation pattern at this range of temporal aggregation periods. We do see that the prediction bands overlap where the respective ranges of both datasets almost meet. For aggregation periods larger than $1 \mathrm{~h}$ the confidence band for the Wageningen dataset becomes so wide as to render the fit meaningless. Therefore, no fitted models for the Wageningen dataset are shown in Fig. 3.2g-i. 

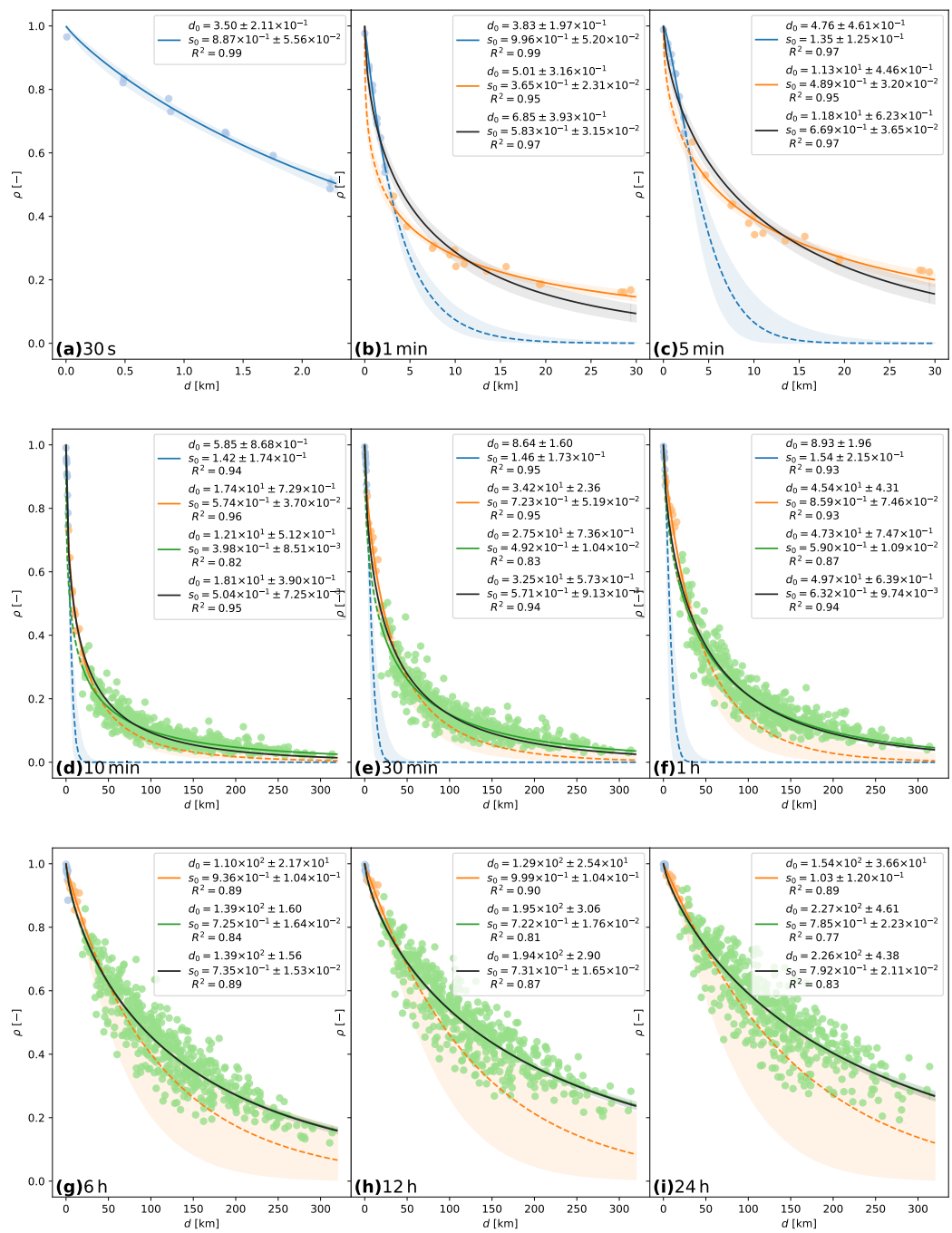

Figure 3.2: Correlograms for different aggregation intervals. Blue dots indicate disdrometer pairs from the Wageningen dataset, while orange dots indicate disdrometer pairs from the Rotterdam dataset and green dots indicate gauge pairs from the nationwide dataset. The correspondingly colored lines indicate the fitted models using only one of the datasets. The fitted model using all datasets is shown in black (in panel b an c the nationwide dataset is not included). Confidence intervals are indicated by the lighter colored bands and extrapolation of the fitted models beyond the range of measurements on which they are based are indicated with dashed lines. The aggregation intervals are from left to right and from top to bottom: $30 \mathrm{~s}, 1 \mathrm{~min}, 5 \mathrm{~min}, 10 \mathrm{~min}, 30 \mathrm{~min}$, $60 \mathrm{~min}, 6 \mathrm{~h}, 12 \mathrm{~h}, 24 \mathrm{~h}$. 

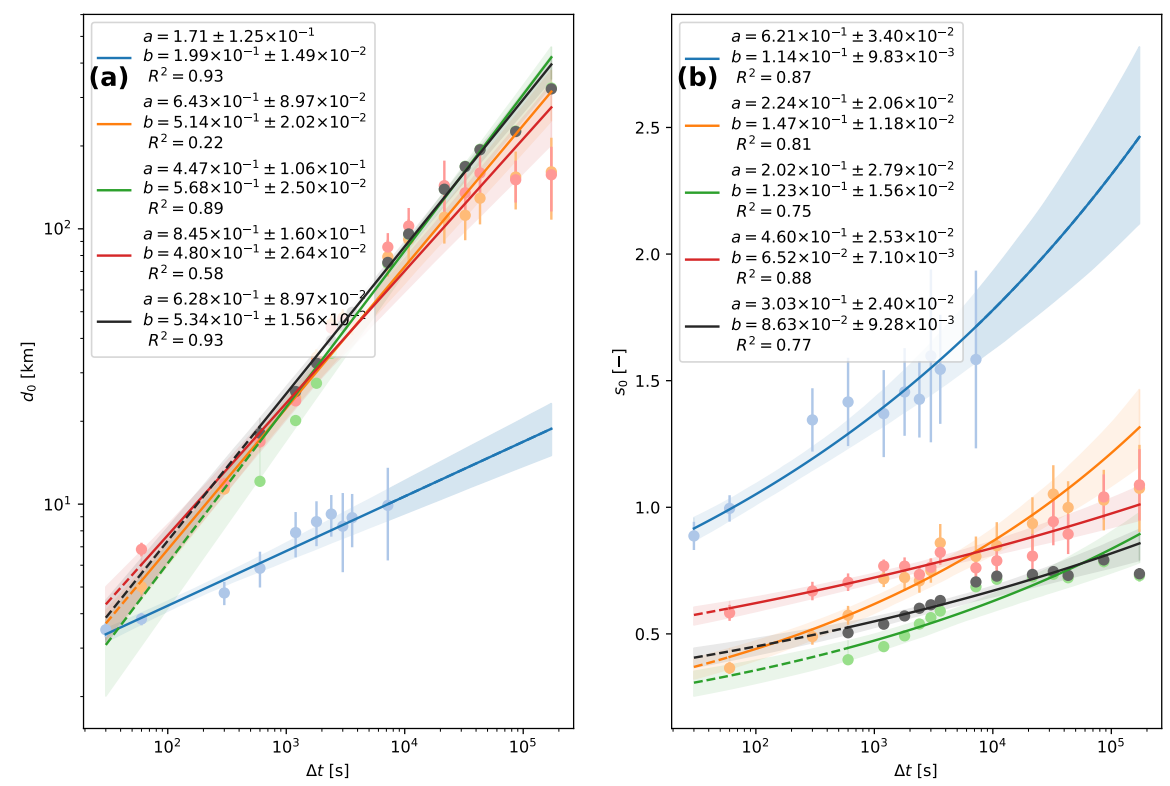

Figure 3.3: a): The decorrelation distance versus the aggregation interval using the Wageningen dataset in blue, the Rotterdam dataset in orange and the nationwide dataset in green. Also included is the decorrelation distance fitted using both the Rotterdam and the Wageningen datasets in red and the decorrelation distance fitted using all datasets in black. b): Same as (a) except showing the shape factor as a function of aggregation interval.

Fig. 3.2d-i also shows the correlograms for the nationwide dataset. Shape factors are lower than for the Rotterdam dataset at all aggregation levels. Decorrelation distances are lower than for the Rotterdam dataset at aggregation levels of $30 \mathrm{~min}$ and below and decorrelation distances are higher at higher aggregation levels.

Because the range of distances covered by the Wageningen and Rotterdam datasets is complementary, we increase the representativeness of the fit by combining the two in a single regression. In doing so, we implicitly assume both datasets to be sampled from the same population: i.e., that there is no climatological difference between the sampled areas and times, and that there are no instrumental differences that could in any way influence the correlations between stations. Although the two datasets are collected in two different time frames, at different locations and with different instruments, the assumption is reasonably justified: the timespan of the datasets is relatively long compared to the aggregation intervals ( 9 months and 1.5 years), they take place within 4 years of each other and both are collected over similar (flat, urban) terrain within $100 \mathrm{~km}$ of each other 
within a larger area which has no significant height differences or mountain ranges. Furthermore, both types of instruments operate along the same general principles: both are optical laser disdrometers.

With that justification, we determine decorrelation distances using correlations found in both datasets, which are shown in Fig. 3.2b and c, and are compared to the regression curve based on only the Rotterdam dataset. Both the shape parameter $s_{0}$ and decorrelation distance $d_{0}$ become higher for the 1 minute aggregation period. For aggregation periods of 5 minutes or above the difference in $d_{0}$ is not significant at a $95 \%$ significance level. For 20 minute aggregation intervals and longer (not shown here, tested up to $1 \mathrm{~h}$ ) the confidence bands overlap over the entire range. Therefore, we can conclude that the rainfall decorrelation pattern observed from just the Rotterdam network is fundamentally in agreement with the pattern observed from both networks. To assess the representativeness of the functions fitted to the combined dataset for both long and short distances, we calculate the coefficient of determination of the function fitted to both datasets applied to the Wageningen dataset or the Rotterdam dataset alone. This yields for e.g. 10 min intervals $R^{2}=0.92$ for Rotterdam and $R^{2}=0.72$ for Wageningen. Therefore this function does accurately describe the decorrelation pattern at smaller $(d<2.3 \mathrm{~km})$ and intermediate $(3.2 \mathrm{~km}<d<30 \mathrm{~km})$ distances.

Similarly, we also fitted the decorrelation model using all three datasets. The same justification applies, except the nationwide dataset is measured with automatic gauges instead of laser disdrometers, which weakens the justification somewhat. The results are illustrated in Fig. 3.2d-i. The decorrelation distances for the aggregation levels of $10 \mathrm{~min}$ and $30 \mathrm{~min}$ are closer to those determined using only the Rotterdam dataset, while at aggregation levels of $1 \mathrm{~h}$ and longer they are not significantly different from those determined using only the nationwide dataset. As before, we assess the representativeness of the parameterization at different distance ranges by calculating coefficients of determination of the fitted function applied to each individual dataset at the $10 \mathrm{~min}$ aggregation level. This gives a reasonable goodness-of-fit for the large $(d>20 \mathrm{~km})$ and intermediate distances ( $R^{2}=0.94$ for Rotterdam and $R^{2}=0.77$ for nationwide), but a failed fit for small distances $\left(R^{2}=-0.94\right)$. Therefore, this provides no evidence that a single parametrization of the extended exponential model can apply to the entire distance range.

Fig. 3.3 shows the fitted model parameters for a larger number of aggregation levels. The relationship between the decorrelation distance and the aggregation interval takes the form of a power-law,

$$
d_{0}=a \Delta t^{b}
$$

where $\Delta t$ is the aggregation period and $a$ and $b$ are fitted parameters. The parameters are given in Fig. 3.3a. A similar power-law is also used to fit the shape factor as a function of the aggregation interval and is given in Fig. 3.3b. The decorrelation - aggregation level relationships obtained using most combinations of datasets are consistent, with confidence bands overlapping over most of the range. The relationship obtained using 
only the Rotterdam dataset results in the lowest goodness-of-fit $\left(R^{2}=0.22\right)$, while the relationship obtained using all three datasets results in the highest coefficient of determination $\left(R^{2}=0.93\right)$. The exception is the relationship obtained using only the Wageningen dataset. The decorrelation distances at the $30 \mathrm{~s}$ level are consistent with the extrapolation obtained from the other fits, but at higher aggregation levels the decorrelation distances are all significantly lower than would have been obtained using any other combination of datasets, up to an order of magnitude difference. The shape factor - aggregation level relationship is not as consistent between the different combinations of datasets. The difference between the fit obtained using all datasets and that obtained using only the nationwide dataset is small but still significant. In all cases and at all aggregation levels the shape factor remains below 1 . The exceptions are the shape factors obtained using only the Wageningen dataset, which are all close to or above 1. In summary: using only the sub-pixel dataset results in anomalous decorrelation parameters, which are inconsistent with the findings from a larger range of scales (both including and excluding the sub-pixel range itself). This could be either due to the limited number of datapoints or the limited range.

We also investigated the effect of using a moving average instead of a sequential averaging scheme for aggregating to longer time intervals. This did not make a significant difference in the results.

In the analyses of the rest of this chapter we will only consider the results obtained using all datasets to fit the model.

\subsubsection{Seasonality of spatial correlation}

In order to investigate seasonal effects in a crude fashion we have performed the abovementioned analyses on two subsets of our data. One subset spanning only the months of December, January and February (winter) and another subset spanning only the months of June, July and August (summer). Because the Wageningen dataset spans only nine months, we performed the analyses only on the Rotterdam dataset and the nationwide dataset. The results for the summer months are very similar to those found for the entire year, while the results for the winter months are very different. Our results show that typical decorrelation distances are far lower in summer than in winter, as can be seen in Fig. 3.4a. This is especially true of the smaller aggregation intervals, while the differences become gradually less towards the larger aggregation intervals. At an aggregation interval of $48 \mathrm{~h}$ there is no significant difference anymore and at even longer aggregation intervals (not shown here) summer decorrelation distances become larger than winter decorrelation distances. However, it should be noted that at these aggregation levels the estimated $d_{0}$ becomes longer than the longest distance over which we have measured. Therefore, this is not necessarily a meaningful result.

There is a striking difference in the values of $s_{0}$ as well as in their relation with aggregation interval found for the winter months and those found for the summer months (Fig. 3.4b). In the summer (and over the entire year) the shape factors all stay below 

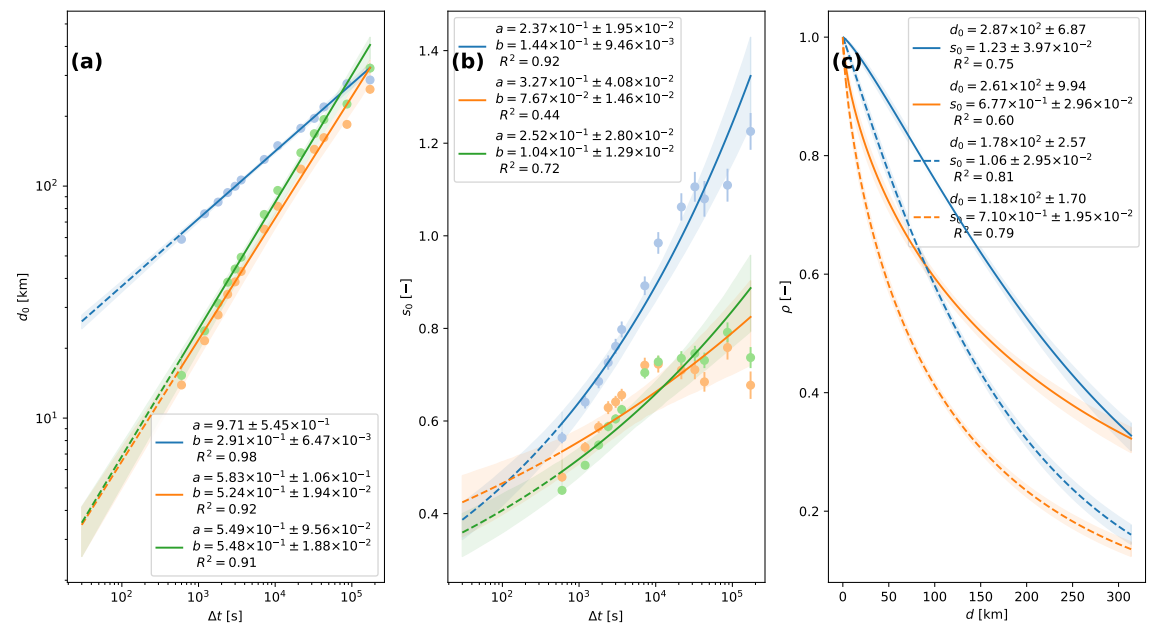

Figure 3.4: a: Decorrelation distance of the disdrometer/gauge pairs versus aggregation interval per season. summer months are indicated in orange, while winter months are indicated in blue. All year values are indicate in green. $b$ : Shape factors of the decorrelation function as a function of aggregation interval per season. Colors are the same as in (a). c: Correlation coefficient as a function of interstation distance. solid lines are based on rainfall aggregated to $48 \mathrm{~h}$, while dashed lines are bases on 6 h aggregated rainfall. Colors as in (a).

approximately 0.8 and they generally do not increase anymore from aggregation intervals of $2 \mathrm{~h}$ onward. As such, the relationship in the summer deviates from the assumed power-law relationship, which is also evident from the goodness-of-fit $\left(R^{2}=0.44\right)$. For the winter months, the shape factor is not only larger than for the summer months, it also increases faster and does not seem to approach an asymptote. Instead, it more closely follows the power-law relationship $\left(R^{2}=0.92\right)$ and it becomes larger than unity for aggregation intervals of $2 \mathrm{~h}$ and larger. In this context, the fact that the decorrelation distances between summer and winter approach each other as the aggregation interval increases can be a bit misleading; for aggregation intervals up to $48 \mathrm{~h}$, the correlation during winter is significantly higher than during summer for the entire distance range. This is illustrated in Fig. 3.4c for aggregation intervals of $6 \mathrm{~h}$ and $48 \mathrm{~h}$.

\subsubsection{Intermittency}

The datasets contain many periods where rain was registered at one or more stations but some other stations were dry at the same time. In addition, there were periods where rain was registered at only certain sub-intervals within a larger temporal aggregation interval. In order to asses the relative effect of such intermittency in rainfall versus the purely quantitative variability in rainfall intensity we have performed the same analysis 

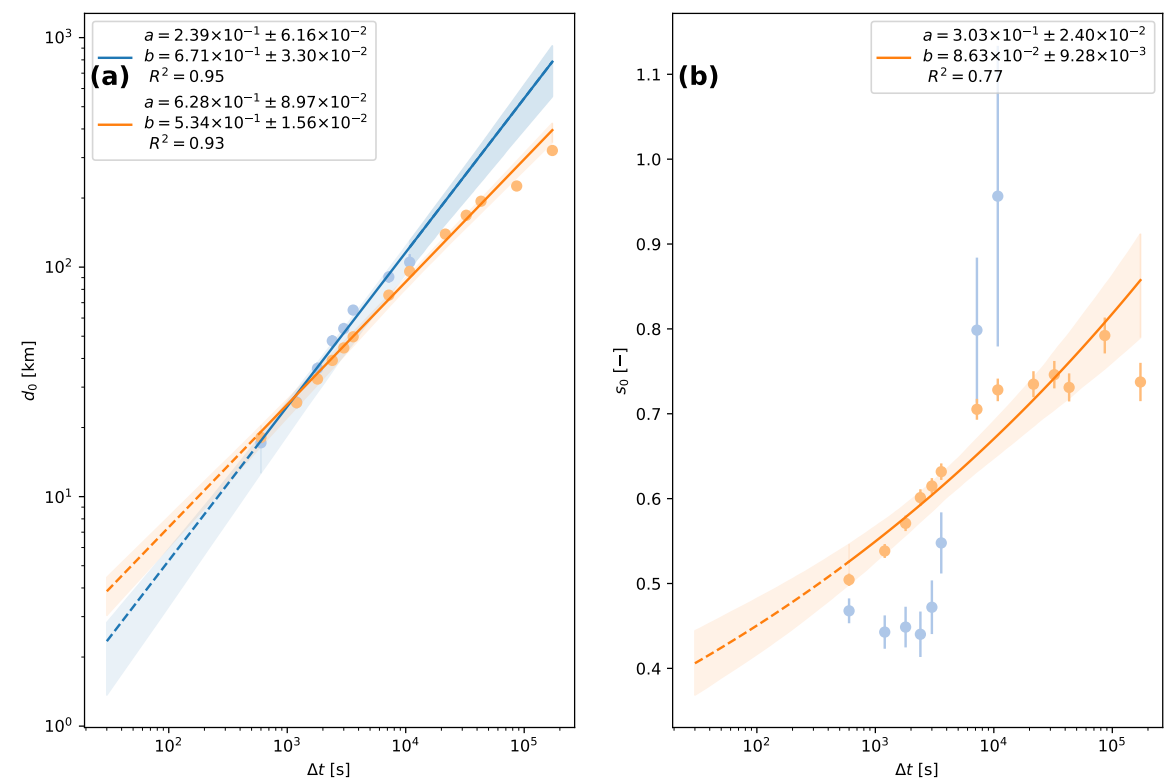

Figure 3.5: a): Dependence of decorrelation on aggregation interval using only the wet intervals. Blue lines and dots indicate estimates using only purely wet intervals for all datasets, while orange includes partially wet intervals as well. b): same as (a) except for the shape factor as a function of aggregation interval.

as described above, but with an extra filtering step. The goal is to exclude any period containing such edge cases. In order to do so we filter out any time intervals per pair of stations where at least one of the two stations for which the correlation coefficient is calculated has reported a rain intensity of $<0.1 \mathrm{~mm} \mathrm{~h}^{-1}$ during any sub-interval. Filtering in this manner leaves $5.0 \%$ of the 1 minute pair-intervals in the Wageningen dataset. Pairintervals where both disdrometers register rainfall intensities $<0.1 \mathrm{~mm} \mathrm{~h}^{-1}$ comprise $92.5 \%$ of the dataset and pair-intervals where at least one of the disdrometers registers rain at at least one subinterval but at least one disdrometer does not do so continuously or at all amount to $2.5 \%$. For the Rotterdam dataset these percentages are $5.0 \%, 89.5 \%$ and $5.5 \%$ respectively.

The decorrelation distances and shape factors calculated from the filtered data are shown in Fig. 3.5 for several aggregation intervals together with the same parameters calculated from the unfiltered dataset. parameters derived from the filtered dataset are not included for aggregation intervals of $6 \mathrm{~h}$ and longer; the number of individual intervals that are completely wet are too few to derive meaningful statistics from. When filtering the data- 
sets in this manner it can be observed that the decorrelation distances are significantly longer as compared to the unfiltered dataset at aggregation levels of $30 \mathrm{~min}$ and longer. However, the distance-time powerlaws have overlapping significance bands over almost the entire range, only diverging at intervals levels of $2 \mathrm{~h}$ and longer. In contrast, the differences in terms of the shape factors are more fundamental. The shape factor-aggregation level relationship in the filtered case is not at all similar to the non-filtered case and cannot be described with a power-law function.

To gain more insight into the spatial and temporal dependancy of the intermittency we use the intermittency ratio, $I$, introduced in Eq. 3.4. The results are plotted against the distance between the stations in Fig. 3.6a. A two-parameter exponential model similar to that used to fit the decorrelation function is also used to fit the distance dependency of the intermittency ratio. The same analysis is performed for different aggregation intervals. The resulting $e$-folding distances and shape factors are shown in Fig. 3.6b-c. A power-law relationship between the aggregation interval and the decorrelation distance is used. No power-law relationship was fitted between the shape factor and the aggregation interval, since it does not provide a good fit.

We see that at e.g. the 10 min scale the conditional probability that two stations at a relative distance $d$ both detect rainfall, given that one of them has detected rainfall is 1 , for $d=0 \mathrm{~km}$ decreasing to 0.1 for $d=300 \mathrm{~km}$. The exponential decrease model fits the observed pattern very well $\left(R^{2}=0.99\right)$. The e-folding distance (analogous to the decorrelation distance) is of the same order of magnitude as the decorrelation distances observed for the same aggregation interval and data selection, but still significantly longer. At longer aggregation intervals, the intermittency ratios become gradually higher. E.g. at a $24 \mathrm{~h}$ aggregation interval the intermittency ratio at $d=300 \mathrm{~km}$ becomes 0.35 . The $e$-folding distance at longer aggregation intervals becomes lower than the decorrelation distance at the same intervals, but still of the same order of magnitude. For all aggregation intervals, the shape factor is lower than 0.5 . The power-law functions fits the efolding distance - aggregation interval relationship quite well $\left(R^{2}=0.86\right)$. Note that the first few aggregation intervals shown in Fig. 3.6b and c provide parameters derived only from the Rotterdam and Wageningen datasets and are therefore not included in the power-law fit. The shape factor-aggregation interval relationship does not conform to a power-law, although some continuous pattern does appear.

The seasonal effect is less pronounced in terms of the intermittency ratios (shown in Fig. $3.6 \mathrm{~b}-\mathrm{c}$ ) than in terms of the rainfall intensities. However, there is still a significant increase in e-folding distance in winter compared to summer. Longer distances in winter are expected given the prevalence of stratiform over convective rainfall. Curiously, the distances are actually lower in the winter when aggregated to intervals of 24 hours or 48 hours. (The fitted trend is not significantly different though). 

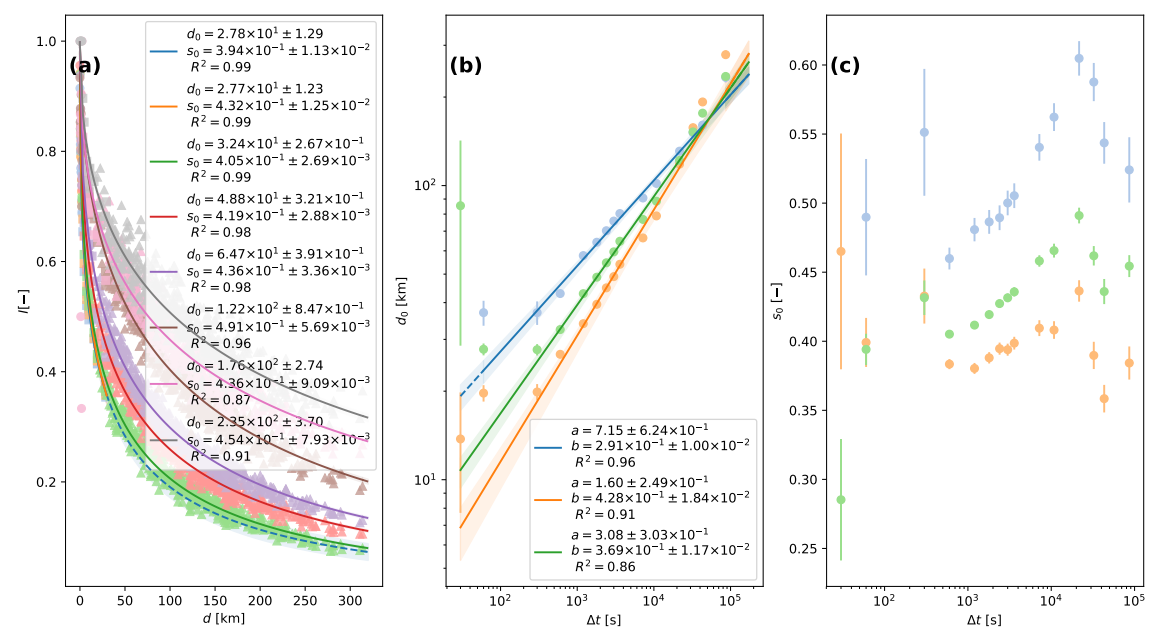

Figure 3.6: a): Intermittency ratios of the disdrometer/gauge combinations plotted against distance for several temporal integration intervals. Circles represent Wageningen disdrometer pairs, while squares represent Rotterdam disdrometer pairs and triangles represent gauge pairs. One shaped exponential function is fitted through all datasets. b): $e$-folding distance of the intermittency ratios of the disdrometer pairs plotted against the aggregation intervals. Parameters determined using all the data are plotted in green. Parameters determined for only the summer months are indicated in orange, while blue shows the parameters determined for only the winter months. c): Shape factor of the intermittency ratio-distance relationship plotted as a function of aggregation interval. Colors are the same as (b).

\subsection{Discussion}

In comparing our findings to the existing literature, a few salient points can be identified: contrary to some other studies we have not included a nugget parameter in our parametric model. Given our relatively small number of point-pairs we wanted to limit the number of degrees of freedom in out model. We find that the inclusion of a nugget parameter does not improve the goodness-of-fit and we find that the nugget is not significantly different from 1. Ciach and Krajewski (2006) estimated the nugget parameter from collocated rain gauges and estimate a nugget for their experiment of 0.996 at a 1-minute integration interval (and even higher at larger intervals), which supports our assertion that the nugget can be neglected. Tokay and Öztürk (2012) also used collocated rain gauges to estimate the nugget parameter; They found a value of 0.97 for their lowest aggregation interval of 5 minutes. Jaffrain and Berne (2012) estimated the nugget from a collection of point-pairs with distances that are all greater than $100 \mathrm{~m}$, which makes their estimation more prone to error. Nevertheless, they do not report nugget parameters lower than 0.92. Peleg et al. (2013) use the same method and get similar results. Villarini et al. 
(2008) however report far lower nugget parameters; 0.51 for 1-minute aggregation intervals, which is in disagreement with the abovementioned reports as well as this study. This may be the result of the lack of point-pairs below 500 m distance in their study. Van de Beek et al. (2011) neglect the nugget as well, however, this is not a fair comparison since they did not consider aggregation intervals shorter than one hour or short distances.

In all the existing literature a roughly power-law relationship is found between decorrelation distance and aggregation interval. We find decorrelation distances that are in good agreement with Ciach and Krajewski (2006) for short aggregation intervals, yet for larger intervals the decorrelation distances found in this study are considerably longer, see Table 3.1. The decorrelation distances found by Villarini et al. (2008) remain in reasonable agreement with our findings throughout the range of time scales. Conversely, the decorrelation distances found by Jaffrain and Berne (2012) and Peleg et al. (2013) are significantly shorter at all aggregation intervals. Jaffrain and Berne (2012) did a separate analysis for convective and frontal rainfall and found that decorrelation distances for frontal rainfall can be roughly 2 to 6 times longer than for convective rainfall, yet still shorter than what we find. Of the studies we have compared only Tokay and Öztürk (2012) reported decorrelation distances that where significantly longer than in our study (almost by a factor of 2 across aggregation intervals).

We find shape factors, $s_{0}$, that are below 1 at all integration intervals and increasing with correlation distance, which is in agreement with Villariniet al. (2008) and Tokay and Öztürk (2012), but counter to the findings of Ciach and Krajewski (2006) and Jaffrain and Berne (2012), who report shape factors above 1 in all cases and most cases, respectively (see Table 3.1). All studies in this comparison reported an increase of the shape factor with aggregation interval except for Peleg et al. (2013), who found shape factors ranging between 0.9 and 1.2 but no trend.

It should be noted that of these studies only Villarini et al. (2008) measured across distances greater than $5 \mathrm{~km}$. As we have shown in Fig. 3.2, not taking into account longer interstation distances, at least of the same order as the decorrelation distance itself, can severely distort the parameter estimates of $d_{0}$ and $s_{0}$. If we take only into account the data from Wageningen, then our $d_{0}$ estimates are more in line with those found by Peleg et al. (2013), while our $s_{0}$ estimates are all around or higher than 1 , increasing at first and from aggregation intervals of $15 \mathrm{~min}$ onwards varying roughly around 1.5.

The longer decorrelation distances that we find in winter can be explained by the prevalence of frontal over convective systems in The Netherlands during winter; the opposite is generally true in summer. Van de Beek et al. (2011) also investigated seasonal differences in rainfall spatial variability in the Netherlands. They analyzed the variability in terms of semivariance, so we cannot directly compare the magnitude of the model parameters. However, they found a range parameter that is much smaller during summer than during winter, which is consistent with our findings. Seasonal differences in rainfall spatial variability have not been investigated extensively at the small scales. Tokay and Öztürk (2012) briefly discuss seasonal differences, but do not provide actual parameter values, making 


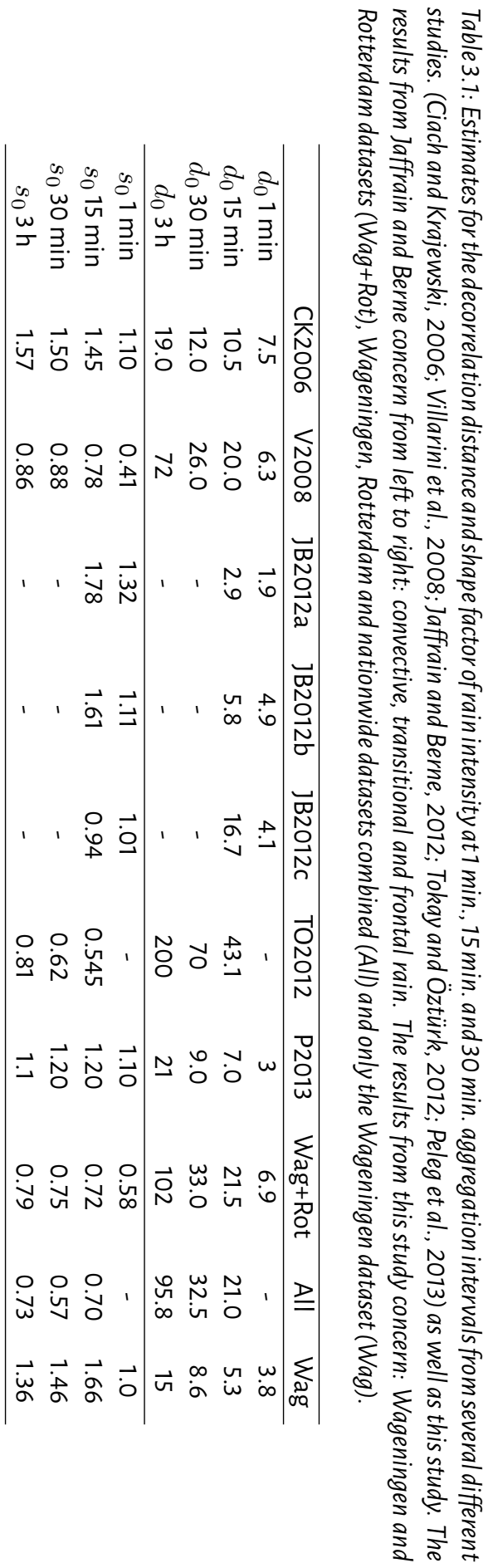


it hard to compare our results to theirs. They investigated two years and found that correlations in winter are higher than in summer during the first year, while correlations in winter were slightly lower than in summer in the second year. They also found large differences between the same seasons in different years. This is quite different than our results, part of which may be due to climatological differences between Virginia, USA and The Netherlands. Both The Netherlands and Virginia are northern hemisphere coastal regions; however, while the climate in Virginia can be classified as humid subtropical, The Netherlands have a temperate maritime climate. Furthermore, the remnants of tropical cyclones can contribute to out-of-season rainfall patterns in Virginia (Tokay and Öztürk, 2012), whereas when such remnants arrive in The Netherlands they are already much weaker.

Habib and Krajweski (2002) and Villariniet al. (2008) did investigate intermittency of rainfall. However, they considered the probability of a rain gauge registering zero rainfall within an area where non-zero rainfall was measured. This is a different metric than we use and not immediately comparable.

\subsection{Conclusions}

We have used data from three different gauge/disdrometer networks at three different spatial scales in the same general area to assess the spatial variability of rainfall. We use decorrelation distance and intermittency ratio as metrics and fit these to an extended exponential model. By combining datasets we obtain a more accurate regression that captures the decorrelation pattern over a broad range of scales. Spatial variability patterns at the small scale are in general agreement with the pattern at larger scales. We confirm that decorrelation distance is related to the temporal aggregation interval by a power law relationship, $d_{0}=0.63 \Delta t^{0.53}$. In winter, the decorrelation distance tends to be significantly higher and follows a different power-law relationship, $d_{0}=9.7 \Delta t^{0.29}$. In general our estimates of the parameters of an exponential decorrelation function of rainfall are in reasonable agreement with estimates by Villariniet al. (2008), but not with other studies that only took into account interstation distances smaller than $5 \mathrm{~km}$. We found that the correlation between stations at the sub $10 \mathrm{~m}$ scale is 1 . This is in agreement with other studies which measured correlations between collocated stations. We have analyzed the spatial patterns of intermittency in rain separately from the spatial pattern in rainfall intensity. The spatial decorrelation pattern of rainfall intensity within contiguous patches of rain is not significantly different than the decorrelation pattern including dry zones within a larger rain field.

Our results show that in order to accurately determine spatial decorrelation distance of rainfall a gauge/disdrometer network with mutual distances of only a few kilometer is not enough. This is demonstrated by the fit obtained using only the Wageningen dataset and is supported by our assessment of other studies. The inclusion of pairs with a mutual distance in the order of tens of $\mathrm{km}$ is sufficient for sub-hour time scales. Nevertheless, we also find that, when taking into account the full scale range from micro- $\gamma$ to meso- $\beta$, 
the the shape of the fitted correlogram does not adequately capture the decorrelation pattern at the micro- $\gamma$ scale, hinting that the modified-exponential model might not be general enough to capture the true shape. Finding a better model could be an avenue for further research.

The spatial pattern in intermittency ratio could be further employed to provide guidelines for the maximum spatial range between which measurement stations may be compared for quality control purposes. For example, in the case of personal weather stations, the range so determined may be used to find with which surrounding stations the PWS must be compared (De Vos et al., 2017). 


\title{
Chapter 4
}

\section{Estimating raindrop size distributions using microwave link measurements}

\begin{abstract}
We present a novel method of using two or three collocated microwave link instruments to estimate the three parameters of a gamma raindrop size distribution (DSD) model. This allows path-average DSD measurements over a path length of several kilometers as opposed to the point measurements of conventional disdrometers. Our model is validated in a round-trip manner using simulated DSD fields as well as a five laser disdrometers installed along a path. Different potential link combinations of frequency and polarization are investigated. We also present preliminary results from the application of this method to an experimental setup of collocated microwave links measuring at $26 \mathrm{CHz}$ and $38 \mathrm{GHz}$ along a $2.2 \mathrm{~km}$ path. Simulations show that a DSD retrieval on the basis of microwave links can be highly accurate. We found that a triple link retrieval provides no added benefit over a dual link retrieval. In practice, the accuracy and success of the retrieval is highly dependent on the stability of the base power level.
\end{abstract}

This chapter is based on: Van Leth, T.C., Overeem, A., Leijnse, H. and Uijlenhoet, R., Estimating raindrop size distributions using microwave link measurements, Atmos. Meas. Tech Discussions, submitted. 


\subsection{Introduction}

The use of microwave links to measure rainfall intensity has received significant attention in the last decade. The main driver for this has been the insight that the backhaul links of mobile communication networks are suitable for such measurements and are available in greater numbers than dedicated rainfall measurement stations in many countries (Messer et al., 2006; Leijnse et al., 2007a; Cosset et al., 2016). In addition, efforts have been taken to expand the range of atmospheric phenomena that can be measured with microwave links including fog (David et al., 2013), solid precipitation (Cherkassky et al., 2014) and evapotranspiration (Leijnse et al., 2007b). Another enticing possibility is the use of multiple link instruments along the same path to measure not just the bulk rainfall intensity, but the path-average raindrop size distribution (DSD) (Rincon and Lang, 2002).

DSD estimates can be used to derive all other bulk rainfall variables and are therefore valuable for a variety of purposes (see e.g. Uijlenhoet and Stricker (1999) for an overview of relevant statistical moments). Examples include precipitation microphysics (e.g. Uijlenhoet et al. (2003)), soil erosion by rain (e.g. Angulo-Martínez and Barros (2015); Salles et al. (1999); Salles and Poesen $(1999,2000)$ ) and radar validation (e.g. Hazenberg et al. (2014)). The use of microwave links for such purposes promises measurements that are more spatially representative of radar or satellite pixels than what is offered by the usually sparse networks of impact, laser and video disdrometers that are currently the most common way DSDs are measured.

In order to estimate the DSD from a limited number of statistical moments, a parameterization has to be used. The gamma distribution with three parameters provides a good estimate for a wide variety of rainfall events (Ulbrich, 1983). Rincon and Lang (2002) where first to attempt a gamma DSD parameter retrieval using two microwave links with promising results. However, their methods require an a priori parameter estimate in addition to the parameters derived from the two measurements. To the best of our knowledge, no further research has been published regarding DSD estimations using microwave links. However, the adjacent field of polarimetric radar measurement has seen much development (see e.g. Fabry (2015b)) and many different methods to estimate DSDs from polarimetric radar have now been developed and tested.

A handful of different techniques can be identified that have been developed to retrieve DSD parameters from a limited number of polarimetric radar moments: The constrained gamma method developed by Zhang et al. (2001), the $\beta$ method proposed by Corgucci et al. (2002) and double-moment DSD normalization (Raupach and Berne, 2017). Several machine learning approaches have also been used: neural networks (Vulpianietal., 2006), Bayesian regression (Cao et al., 2010) and tree-based genetic programming (Islam et al., 2012).

In this chapter we will explore the potential of a numerical retrieval from microwave attenuation and/or differential propagation phase shift. We consider two different techniques: The first method uses three measured microwave link variables to derive the three 
parameters of the gamma distribution. Here the gamma parameters are weakly constrained (i.e., only a limited range of parameter values is allowed). The other method uses two measured microwave link variables to derive two parameters of the gamma distribution. Here, the third parameter is completely constrained by the other two, similar to the method used by Zhang et al. (2001) for radar moments. We will apply these techniques first using a simulated dataset based on radar and disdrometer measurements from the Ardèche region, France. Next, we will apply the method to microwave link variables derived from a set of path-averaged measurements from five laser disdrometers in Wageningen, The Netherlands. Lastly, we will test the methods on measurements from microwave link instruments along the same path as the disdrometers in Wageningen (see Chapter 2) and compare the resulting DSDs with the DSDs measured from the disdrometers.

The chapter is organized as follows: In Section 4.2 we present in more detail the datasets used in this chapter. In Section 4.3 we present the theory and the methods used to retrieve DSDs from microwave link variables. We will also describe the validation methods employed. In Section 4.4 we discuss the results retrieved from the Ardèche dataset. In section 4.5 we discuss the results of several tests using simulated attenuations from the Wageningen disdrometer dataset. We also consider the efficacy of different frequency combinations and the robustness of the retrieval to measurement uncertainty. In Section 4.6 we will apply the developed methods to actual link measurements that were obtained in Wageningen. In Section 4.7 we will present our thoughts on the feasibility of the techniques in practice and the choices made in this chapter. Finally, in Section 4.8 we come to conclusions and give recommendations for further study.

\subsection{Data}

\subsubsection{Ardèche DSD reanalyses}

Our first test-case consists of 2-dimensional interpolated DSD fields based on polarimetric radar data as well as disdrometer data measured in the Ardèche, France, as part of the HyMeX campaign (Raupach and Berne, 2016). These fields were generated using an advection-based temporal interpolation technique (Raupach and Berne, 2017). The data used here is based on a slightly improved version of that technique and was introduced by De Vos et al. (2018). This field has spatial dimensions of $20 \times 20 \mathrm{~km}$ and a resolution of $100 \mathrm{~m}$. It covers two distinct events on 27 November 2012 and 27 October 2013. Their durations are in the order of several hours and the time step is $30 \mathrm{~s}$. The DSD is divided in 20 diameter bins with unequal bin width based on the detection bins of the OTT Parsivel ${ }^{2}$ laser disdrometer measurements upon which the reanalysis is partially based. The smallest drop diameter class is $0.3 \mathrm{~mm}$, while the largest diameter is $6.5 \mathrm{~mm}$.

We take a transect through this field over the entire length of the field (Fig. 4.1a) and average the DSD over this transect to approximate the footprint of a microwave link. We calculate the attenuations and differential phase shifts for a number of frequencies: $15 \mathrm{CHz}$, $26 \mathrm{CHz}, 32 \mathrm{CHz}$ and $38 \mathrm{CHz}$. This includes the frequencies employed in the Wageningen 

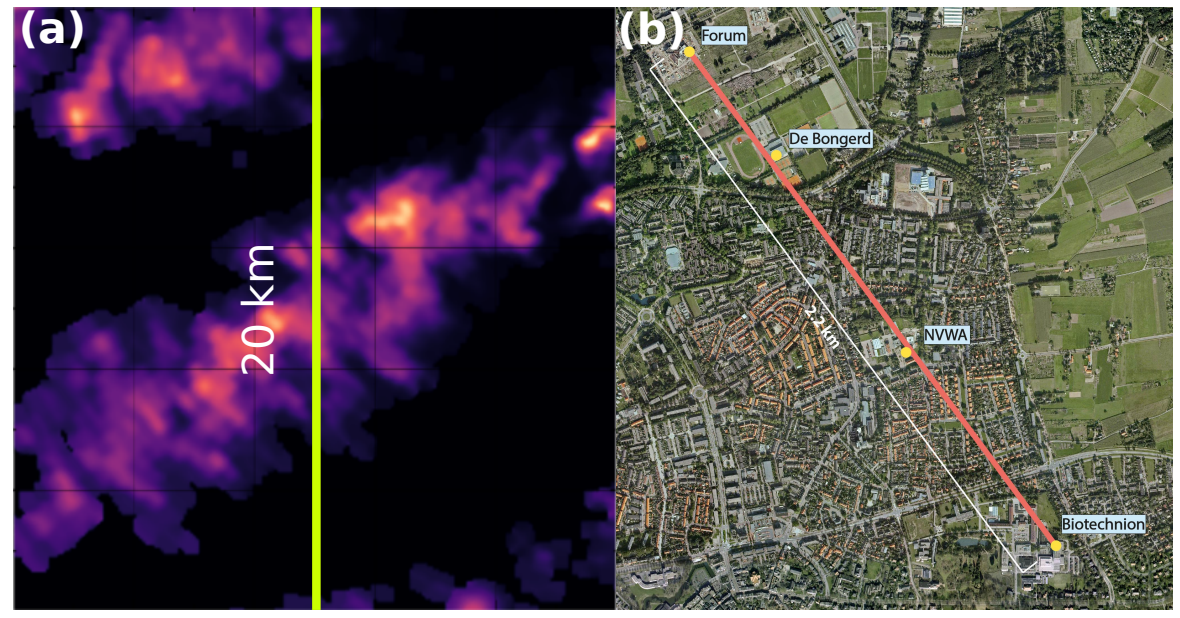

Figure 4.1: a) Sample rain field from the Ardèche dataset with the chosen transect. b) Positions of the disdrometers and the link path in the Wageningen dataset.

experiment (see below) and covers the range of most commonly employed carrier frequencies in mobile phone networks.

In order to calculate the microwave link variables from the DSD data we first need to interpolate the data in the diameter dimension from the irregular bins to a regular diameter grid with a resolution of $0.1 \mathrm{~mm}$. The main reason for this is to accurately reproduce the shape of the scattering cross sections as a function of diameter, as simply assuming the scattering cross section to be constant for the entire $d D$ interval would introduce too much error. We have used a simple linear interpolation method for this purpose.

\subsubsection{Wageningen link experiment}

Our second second test case is a microwave link experimental setup in Wageningen, The Netherlands (Chapter 2 ). The setup consists of three collocated microwave links arranged between two buildings $2.2 \mathrm{~km}$ apart and covering mostly built-up terrain. The setup contains one dual-polarized $38 \mathrm{CHz}$ link, which also measures the phase difference between the two polarizations, one additional single polarized $38 \mathrm{CHz}$ link (not used for this chapter) and a single polarized $26 \mathrm{CHz}$ link. In addition five OTT Parsivel laser disdrometers are positioned at four locations beneath the link path, including the sites of the transmitting and receiving antennas, as shown in Fig. 4.1b. The data used in the following analyses is all taken from the period between 1 April 2015 and 1 January 2016. We also specifically focus on one event on 27 July 2015 for illustrative purposes.

In order to use the disdrometer data to represent the DSD of the link path we take a weighted spatial average over the disdrometer data. As with the Ardèche data, we interpolate the DSD data in the diameter dimension from the irregular bins to regular in- 
tervals before calculating the microwave link variables. In order to improve the robustness of the results, we only consider measurement intervals where each one of the five disdrometers counted at least 50 drops (see Uijlenhoet et al. (2006) on the effect of drop sample size on the robustness of the DSD estimate). We also apply the correction method of Raupach and Berne (2015) to the disdrometer DSDs.

\subsection{Methods}

\subsubsection{Basic procedure}

To determine the underlying DSD from a limited number of statistical moments we need an approximation with a limited number of parameters. One of the most widely used approximations for rain DSD is the gamma distribution suggested by Ulbrich (1983),

$$
N(D)=N_{0} D^{\mu} e^{-\Lambda D}
$$

where $D$ is the drop diameter in $\mathrm{mm}$, and $N_{0}, \mu$ and $\Lambda$ are the parameters determining the shape and drop concentration. The parameter $\mu$ is a dimensionless shape parameter and $\Lambda$ is a slope parameter with a unit of $\mathrm{mm}^{-1}$. Note that the dimension of $N_{0}$ is dependent on the value of $\mu$. Therefore it is convenient to also use a derived parameter,

$$
N_{T}=N_{0} \Lambda^{-(\mu+1)} \Gamma(\mu+1)
$$

where $\Gamma$ is the gamma function. $N_{T}$ has the unit $\mathrm{m}^{-3}$ and is equal to the total drop concentration (assuming integration limits of 0 to $\infty$ ), resulting in

$$
N(D)=N_{T} \frac{\Lambda}{\Gamma(\mu+1)}(\Lambda D)^{\mu} e^{-\Lambda D}
$$

The specific attenuation of a link signal in $\mathrm{dB} \mathrm{km}^{-1}$ at a given frequency can be described in terms of the DSD,

$$
\begin{aligned}
& k_{H}(\lambda)=C_{k} \frac{\lambda^{2}}{\pi} \int_{0}^{\infty} \Im\left[S_{H H}(\lambda, D)\right] N(D) d D \\
& k_{V}(\lambda)=C_{k} \frac{\lambda^{2}}{\pi} \int_{0}^{\infty} \Im\left[S_{V V}(\lambda, D)\right] N(D) d D,
\end{aligned}
$$

where $\lambda$ is the wavelength of the incoming and outgoing waves in $\mathrm{mm}, \Im$ is an operator indicating the imaginary part of its argument and $C_{k}=10^{-3} \ln (10)^{-1} \mathrm{~dB} \mathrm{~m}^{3} \mathrm{~km}^{-1} \mathrm{~mm}^{-2}$ 
is a unit conversion factor. $S_{H H}$ and $S_{V V}$ (dimensionless) are the diagonal components of the forward scattering amplitude matrix $\mathbf{S}$ defined by the relationship

$$
\vec{E}=\mathbf{S} \cdot \overrightarrow{E_{0}}
$$

where $\vec{E}_{0}$ is the electric field strength of the incoming electromagnetic (EM) wave and $\vec{E}$ is the electric field strength of the outgoing EM wave. The specific phase shift (in rad km${ }^{-1}$ ) between the horizontal and vertical components of an outgoing EM wave of a given frequency due to forward scattering can also be described as an integral over the DSD,

$$
\phi(\lambda)=C_{\phi} \frac{\lambda^{2}}{\pi} \int_{0}^{\infty} \Re\left[S_{H H}(\lambda, D)-S_{V V}(\lambda, D)\right] N(D) d D,
$$

where $\Re$ is an operator indicating the real parts instead of the imaginary parts of the diagonal components of the scattering amplitude matrix. $C_{\phi}=10^{-3} \mathrm{~m}^{3} \mathrm{~km}^{-1} \mathrm{~mm}^{-2}$ is another unit conversion factor.

The scattering efficiencies are defined as

$$
\begin{array}{r}
Q_{H}(\lambda, D)=\frac{4 \lambda^{2}}{\pi^{2} D^{2}} \Im\left[S_{H H}(\lambda, D)\right] \\
Q_{V}(\lambda, D)=\frac{4 \lambda^{2}}{\pi^{2} D^{2}} \Im\left[S_{V V}(\lambda, D)\right] \\
Q_{\phi}(\lambda, D)=\frac{4 \lambda^{2}}{\pi^{2} D^{2}} \Re\left[S_{H H}(\lambda, D)-S_{V V}(\lambda, D)\right] .
\end{array}
$$

It is the subtle differences in the shapes of the scattering efficiencies as functions of diameter for different frequencies and polarizations that contain the information necessary to retrieve the DSD. We calculate the scattering amplitude matrix using the T-matrix method (Waterman, 1965) following the approach of Mishchenko et al. (1996); Mishchenko and Travis (1998); Mishchenko (2000). We assume the drops to be oblate spheroids with axis ratios dependent on the drop diameters following the relationship of Thurai et al. (2007) and averaging over canting angles following a Gaussian distribution with a mean of $0^{\circ}$ (vertical) and a standard deviation of $2^{\circ}$. Resulting scattering efficiencies at some relevant frequencies are shown in Fig. 4.2 as a function of volume-equivalent diameter.

The variables used as input in the retrieval could be attenuation of horizontally or vertically polarized radiation or phase differences between horizontally and vertically polarized radiation at one or several frequencies. In order to be able to use attenuations and 


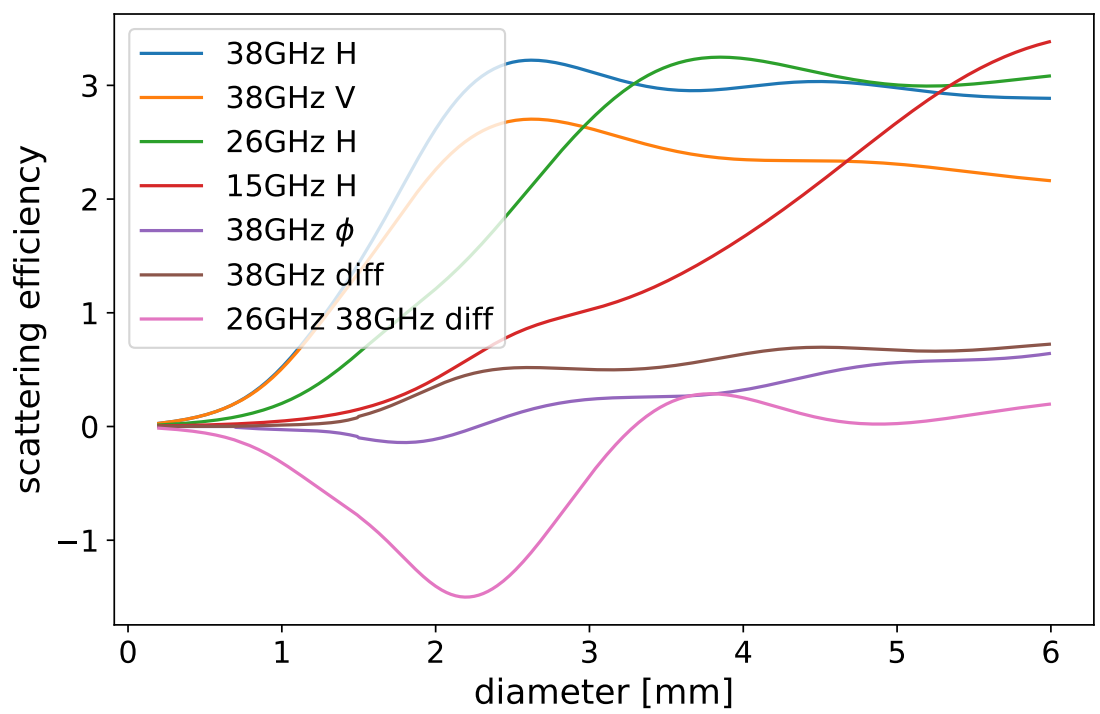

Figure 4.2: Scattering efficiency of raindrops as a function of drop volume-equivalent diameter modeled with the T-matrix method, at a temperature of $288 \mathrm{~K}$.

phase differences interchangeably in the retrieval algorithm we rearrange Eqs (4.4), (4.5) and (4.7) to have the same form.

$$
\begin{aligned}
& k_{H}^{*}=\int_{0}^{\infty} \sigma_{H}(D) N(D) d D \\
& k_{V}^{*}=\int_{0}^{\infty} \sigma_{V}(D) N(D) d D \\
& k_{\phi}^{*}=\int_{0}^{\infty} \sigma_{\phi}(D) N(D) d D,
\end{aligned}
$$

where $k_{H}^{*}=k_{H} / C_{k}, k_{V}^{*}=k_{V} / C_{k}, k_{\phi}^{*}=\phi / C_{\phi}, \sigma_{H}=\frac{\lambda^{2}}{\pi} \Im\left[S_{H H}\right], \sigma_{V}=\frac{\lambda^{2}}{\pi} \Im\left[S_{V V}\right]$ and $\sigma_{\phi}=\frac{\lambda^{2}}{\pi} \Re\left[S_{H H}-S_{V V}\right]$. We will from here on refer to an arbitrary input microwave link variable as $k_{i}$, where $i \in 1,2,3$, such that

$$
k_{i}=\int_{0}^{\infty} \sigma_{i}(D) N(D) d D .
$$



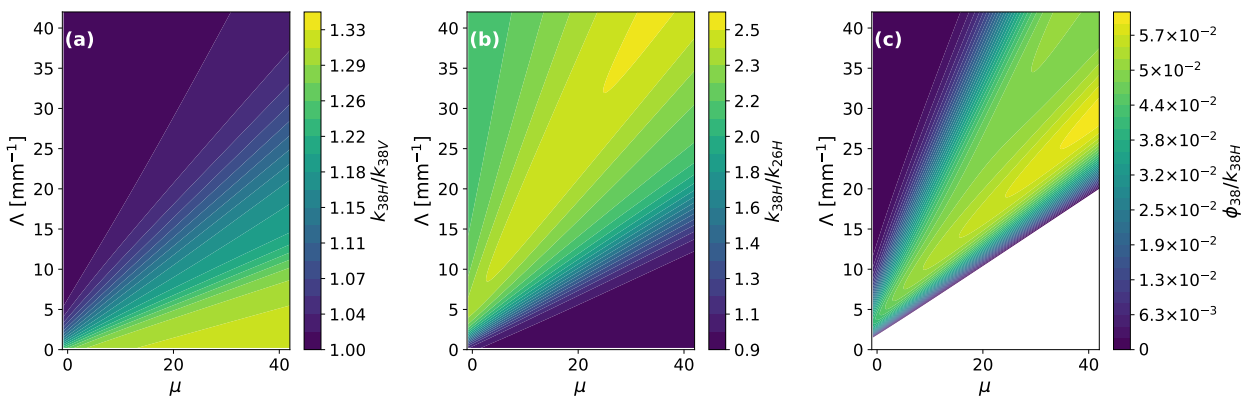

Figure 4.3: Several ratios of microwave link observables that can be used as input to the DSD retrieval, as a function of parameters $\mu$ and $\Lambda$. a) $k_{38 H} / k_{38 \mathrm{~V}}$, b) $k_{38 \mathrm{H}} / k_{26 \mathrm{H}}$ and c) $\phi_{38} / k_{38 \mathrm{H}}$.

\subsubsection{Three-parameter method}

Inserting Eq. (4.1) into Eqs. (4.11) - (4.13) and taking the ratio of two variables we arrive at the following set of equations:

$$
\begin{aligned}
& \frac{k 1}{k 2}=\frac{\int_{0}^{\infty} \sigma_{1}(D) D^{\mu} e^{-\Lambda D} d D}{\int_{0}^{\infty} \sigma_{2}(D) D^{\mu} e^{-\Lambda D} d D} \\
& \frac{k 2}{k 3}=\frac{\int_{0}^{\infty} \sigma_{2}(D) D^{\mu} e^{-\Lambda D} d D}{\int_{0}^{\infty} \sigma_{3}(D) D^{\mu} e^{-\Lambda D} d D}
\end{aligned}
$$

Note that these ratios do not depend on the $N_{0}$ or $N_{T}$ parameter. If we now replace the integrals by discrete summations we can use an iterative nonlinear root-finding technique to find values for $\mu$ and $\Lambda$ from two ratios of microwave moments. Knowing $\mu$ and $\Lambda$, we can directly solve the discretized equation of one of the microwave link variables for $N_{T}$.

Fig. 4.3 shows several possible ratios of microwave link variables as a function of $\mu$ and $\Lambda$. These observables are calculated using the forward model of Eq. (4.15). Because it is possible to detect the attenuation of the horizontal signal and the vertical signal and the phase difference at a given frequency using a single set of antennae, we prefer this combination of variables over combinations of attenuations at multiple frequencies. We can see in Fig. 4.3 that the ratios of $k_{H} / k_{V}$ and $\phi / k_{H}$ provide complementary information and are therefore in principle suitable.

We use the Powell hybrid method to solve the system of equations of Eq. (4.15). We also tested several other gradient-based root-finding methods such as Levenberg-Marquardt and this makes little difference in the stability of the retrieval. The convergence of the retrieval is highly dependent on the initial guess. This is problematic, as we want to automatically retrieve a large number of DSDs without manual input. In many retrieval at- 

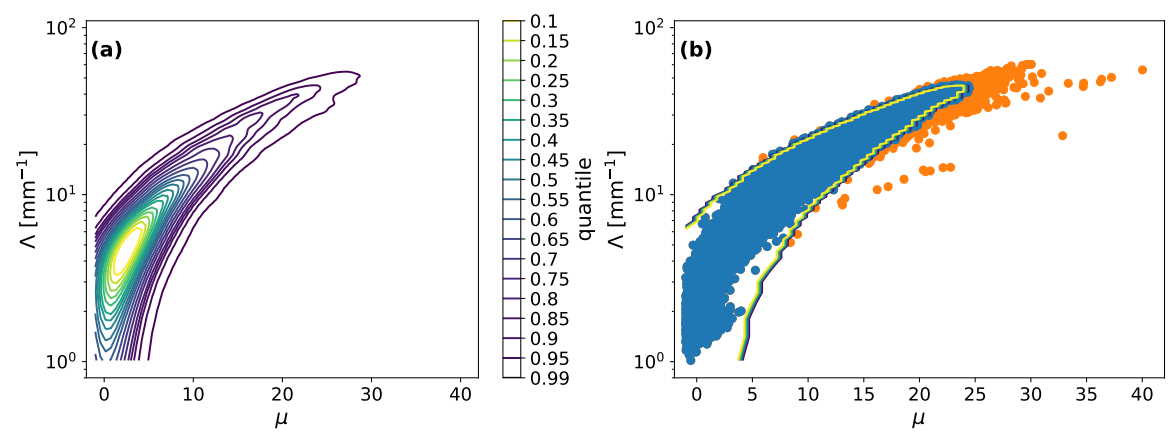

Figure 4.4: a) Density plot of parameter results from the method of moments, where density contours are given as quantiles enclosed within the contour. Results are for the complete 9-month set of path-averaged disdrometer data. b) 0.95 quantile contour overlaid on a scatterplot of all parameter combinations retrieved with the method of moments.

tempts the gradient-based root-finding algorithm diverges towards infinity. In order to prevent this we restrict the root finding algorithm to a limited range of parameter values. If the estimates reach the edge of the range, we reset the parameters to a new initial guess which is taken from within the allowed range, but offset from the first guess by $\Delta \Lambda=0.2 \mathrm{~mm}^{-1}$ or $\Delta \mu=0.2$. We systematically traverse the $\mu-\Lambda$ space in this way starting from $(-1,0)$ until convergence is reached.

Even when the algorithm converges, the solution of the system of equations is not necessarily unique. We need an extra set of constraints to make sure we retrieve the parameters that are the most plausible. In order to do so we use the parameters retrieved by the analytical method of moments of Tokay and Short (1996) as a rough indication of plausible combinations of $\mu$ and $\Lambda$ values. We have calculated the parameters using this method for all individual timesteps in the 9-month record of the spatially weighted average of the disdrometers placed in Wageningen. Figure $4.4 \mathrm{~b}$ shows all these individual retrievals in the $(\Lambda, \mu)$ space. We then apply a kernel density estimator to this dataset (shown in Fig. 4.4a) and calculate the total fraction of data points contained within the contour lines. We then choose the contour corresponding to the 0.95 quantile as a mask. This mask is also shown in Fig. 4.4b.

The mask is then applied to all attenuation/phase-based numerical retrievals to define the range of allowed parameter values. If the estimate is outside this contour, we reset the root-finding algorithm with a new initial guess that is within the contour, but slightly perturbed from the previous initial guess as described above. This is continued until we find convergence within the contour or we reach the maximum number of iterations without a solution. 


\subsubsection{Two-parameter method}

When only two microwave link variables are available the system of equations of Eq. (4.15) is reduced to just one equation:

$$
\frac{k 1}{k 2}=\frac{\int_{0}^{\infty} \sigma_{1}(D) D^{\mu} e^{-\Lambda D} d D}{\int_{0}^{\infty} \sigma_{2}(D) D^{\mu} e^{-\Lambda D} d D}
$$

In order to still solve for the two parameters an additional equation is required for the relationship between $\mu$ and $\Lambda$. We obtain this relationship empirically. We obtain gamma DSD parameter values via the analytical method of moments from the Wageningen disdrometer dataset. We then fit a second order polynomial function (as shown in Fig. 4.5) to the values of $\mu$ and $\Lambda$ with a linear least-squares method. All 9 months of data were used to fit this function. The resulting relationship is

$$
\Lambda=2.5 \cdot 10^{-2} \mu^{2}+1.0 \mu+2.0
$$

which is similar in magnitude but still somewhat different to the relationship found by Zhang et al. (2003). We can substitute this equation for $\Lambda$ in Eq. (4.16) and then solve for $\mu$ using Brent's root finding method. We prefer this method because it is not based on gradients and therefore guaranteed to find a solution if it exists. It is, however, not applicable to multivariate problems.

Figure 4.6 shows the ratio of Eq. (4.16) as a function of $\mu$ for two combinations of microwave attenuations that can be measured with the Wageningen link setup: two polarizations at $38 \mathrm{CHz}$ and two frequencies $(26 \mathrm{GHz}$ and $38 \mathrm{CHz})$ at horizontal polarization. From this we can see that the dual polarization configuration is more suitable for retrieving the DSD; the dual frequency configuration has non-unique solutions for high underlying values of $\mu$ whereas the dual polarization model is monotonously decreasing over the entire range of valid $\mu$ values. On the other hand, the dual-frequency ratio of attenuations is much more sensitive to changes in $\mu$ for $\mu$ between -2 and 8 , potentially yielding more accurate estimates of this parameter. For the dual-polarization ratio (at $38 \mathrm{CHz}$ ) it can also be seen that ratios lower than 1 and higher than 1.25 are not valid. If such ratios are observed they would yield no solution.

\subsubsection{Validation methods}

We test the capability of the methods to accurately retrieve DSDs and their associated statistical moments with two different datasets of measured drop size distributions. We use Eqs (4.11), (4.12) and (4.13) to calculate the microwave link variables from the known DSDs. We then use those variables as input to the retrieval algorithms described in the previous sections to retrieve the parameters of the gamma distribution approximating 


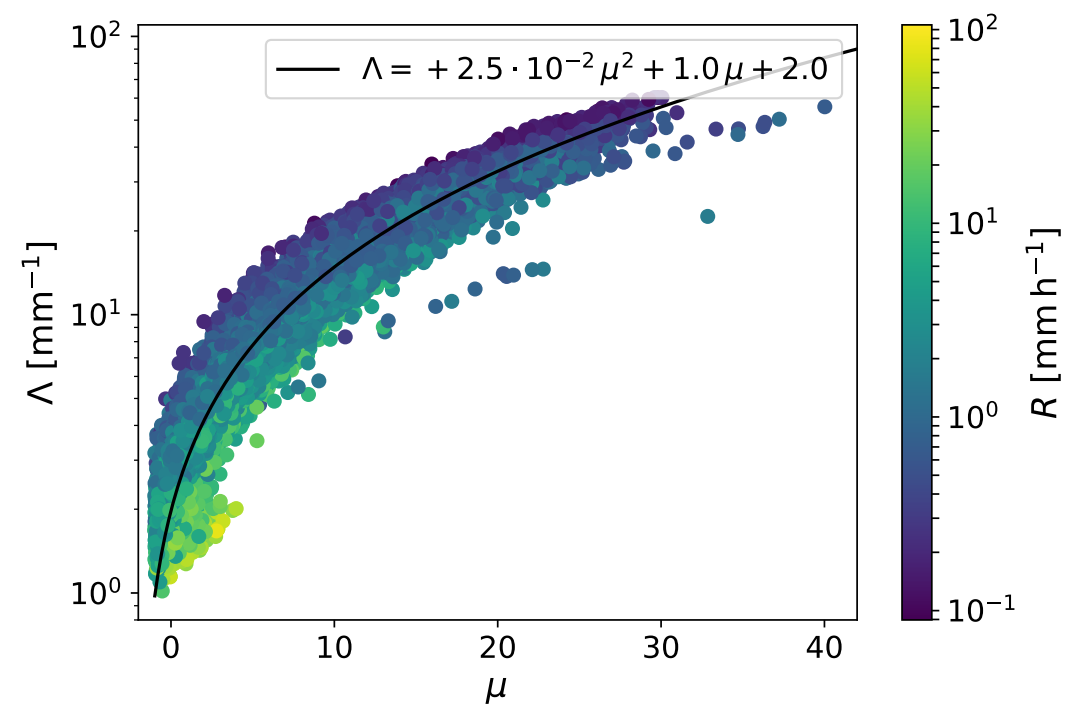

Figure 4.5: Polynomial fit of the $\Lambda$ and $\mu$ parameters in the gamma distribution function fitted to parameter values obtained from the TS96 method of moments. based on the 9 months of pathaveraged disdrometer data from Wageningen. The colors indicate the corresponding rain intensities.

the DSD. From those parameters the complete DSD and its statistical moments are reconstructed. We then compare these to the original DSD and its moments to assess the systematic bias and random error in the retrieval. To be able to distinguish between cases where the gamma distribution is simply not a good fit for the measured DSD and cases where the retrieval itself is the cause for inaccuracies we also calculate the gamma parameters using the analytical method based on integer moments of DSD developed by Tokay and Short (1996) (TS96). These can be used to directly evaluate the parameters themselves as well if we assume the TS96 approach yields the correct parameters.

In order to assess the performance of the retrieval methods we will use a number of statistical measures throughout the results section. As a measure of the accuracy of the retrieval we use the median of the residuals (MOR). As a measure of the precision of the retrieval we use the median absolute deviation of the residuals with respect to the median of the residuals (MAD). We chose MAD and MOR over the use of standard deviation and means, because there where a relatively small number of extreme deviations which would otherwise have too much influence and thus would not give much information about the typical precision. The statistical metrics employed here are less influenced by non-normality and outliers. Because the number and severity of the extreme deviations is also an important part of the performance assessment of the retrieval, we also compute the 95 th percentile absolute deviation with respect to the median of the residuals 


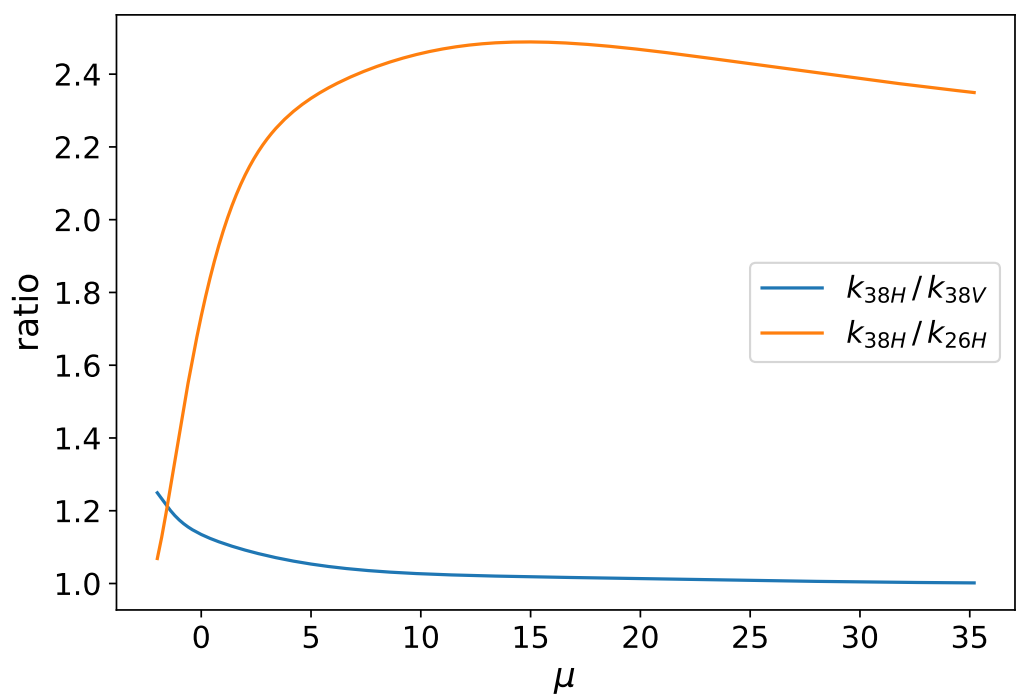

Figure 4.6: Ratios of attenuations as a function of the $\mu$ parameter for the two-parameter retrieval method.

(95AD). Together with the MAD this gives a more complete picture of the distribution of the errors, while still being insensitive to the real outliers. All metrics are normalized with respect to the median of the original quantities.

\subsection{Validation using simulated DSD}

\subsubsection{Single retrieval}

Figure 4.7a shows a typical three-parameter retrieval result using horizontal attenuation, vertical attenuation and phase difference at a single frequency ( $38 \mathrm{CHz}$ in this case). The normalized difference between the retrieved DSD and the original simulation procedure,

$$
\Delta N^{*}(D)=\frac{N_{r}(D)-N_{m}(D)}{N_{T}},
$$

where $N_{r}$ is the retrieved DSD, $N_{m}$ is the originally measured DSD and $N_{T}$ is the integral over all diameters of the original DSD, is illustrated in Fig. 4.7c. $\Delta N^{*}$ is within $10^{-4}$ for particles larger than $1 \mathrm{~mm}$. However, at the smallest sizes the results tend to diverge up to $4.5 \cdot 10^{-3}$. In practice we are often more interested in quantities that scale with the statistical moments of the DSD rather than in the DSD itself. Important quantities are e.g. liquid water content $W$ (3rd order moment), rain intensity $R$ (close to 4 th order) 

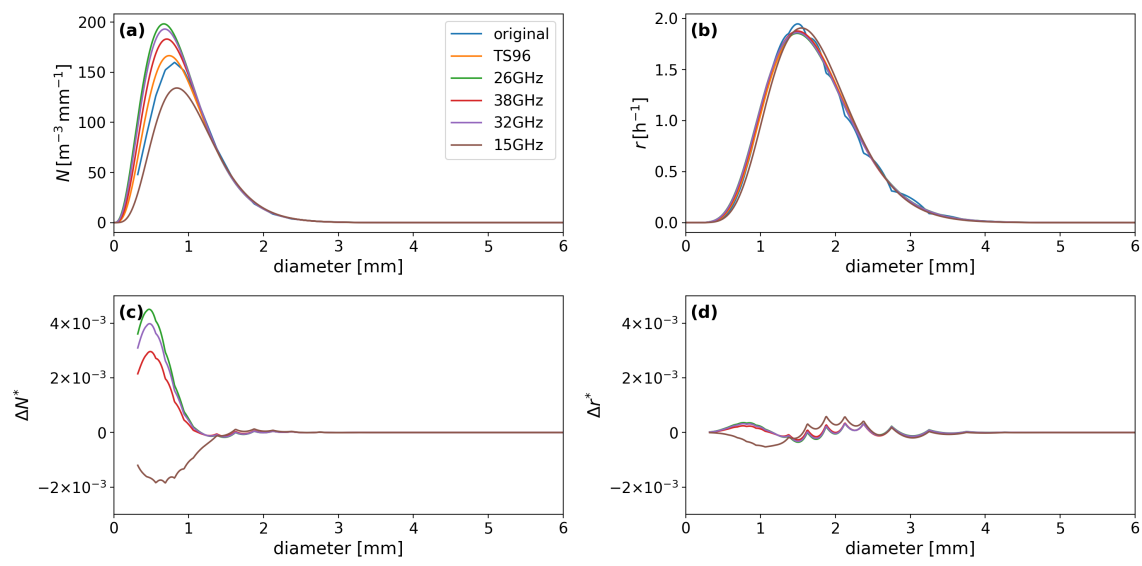

Figure 4.7: a) DSD retrieved from a single timestep in the first Ardèche event using several different microwave frequencies. b) Specific rain intensity for the same timstep. c) Relative difference in DSD compared to the original DSD. d) Relative difference in specific rain intensity compared to original DSD.

and radar reflectivity $Z$ (6th order). For higher order moments, the contribution of the smallest drop sizes decreases. To illustrate this we also show the partial rain intensity as a function of drop diameter,

$$
r(D)=\frac{\partial R(D)}{\partial D}
$$

for the same retrieval parameters in Fig. 4.7b. The normalized difference in the partial rain intensity (illustrated in Fig. 4.7d) is given by

$$
\Delta r^{*}(D)=\frac{r_{r}(D)-r_{o}(D)}{R_{o}}
$$

where $R_{o}$ is the total rain intensity based on the original DSD, $r_{r}(D)$ is the retrieved diameter-specific rain intensity and $r_{o}(D)$ is the original diameter-specific rain intensity. From Fig. $4.7 \mathrm{~d}$ it can be seen that $-10^{-3}<\Delta r^{*}<10^{-3}$ for all drop sizes. The difference in the total drop concentration is $\Delta N_{T}<0.2 \cdot N_{T}$ in the first case, while the difference in the total rain intensity is $\Delta R<0.03 \cdot R_{o}$. The assessment of the accuracy of such a retrieval must therefore take into account its most likely application. We will focus our attention in this section on the rainfall intensity and to a lesser extent on the individual gamma DSD parameters. In further sections, when more detailed comparison is required, we will also look at a range of integer moments. 

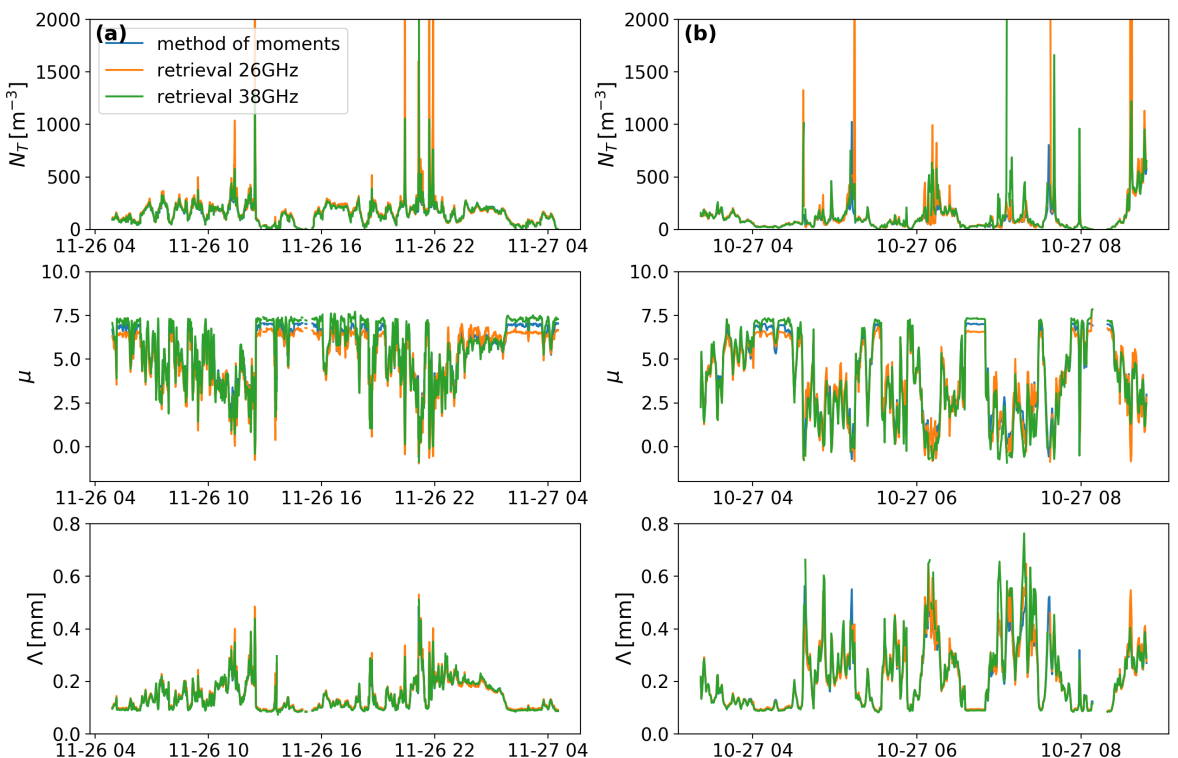

Figure 4.8: Gamma distribution parameters retrieved from the two simulated events from the Ardèche region on 26/27 November 2012 (a) and 27 October 2013 (b). The retrievals are performed using the three-parameter numerical microwave method and the TS96 method is shown as a reference.

\subsubsection{Complete events}

Figure 4.8a shows the results of the 3-parameter retrieval for the first Ardèche event, which took place from 26 November 2012 at UTC 4:54 to 27 November at UTC 4:36. The total duration is almost $24 \mathrm{~h}$ and the precipitation intensity averages at $1.77 \mathrm{~mm} \mathrm{~h}^{-1}$ with a maximum of $10.29 \mathrm{~mm} \mathrm{~h}^{-1}$. The gamma DSD parameters of the retrieval are mostly very close to those of the direct method-of-moments procedure, with a few rather large exceptions, particularly noticeable in the $N_{T}$ parameter. These outliers do not seem to correspond with any particularly high or low precipitation intensity. It can be seen that the temporal evolution of $\mu$ is very close to the temporal evolution of $\Lambda$, with a correlation coefficient of 0.86. Similar results can be observed for the second event (see Fig. 4.8b), which took place on 27 October 2013 from UTC 03:22 to UTC 8:48, with a total duration of $3.5 \mathrm{~h}$, an average intensity of $1.58 \mathrm{~mm} \mathrm{~h}^{-1}$ and a maximum intensity of $27.05 \mathrm{~mm} \mathrm{~h}^{-1}$. The correlation coefficient between $\mu$ and $\Lambda$ is $\rho=0.87$.

Looking at the rainfall intensity over time for both events (Fig. 4.9a and c), we see that the 

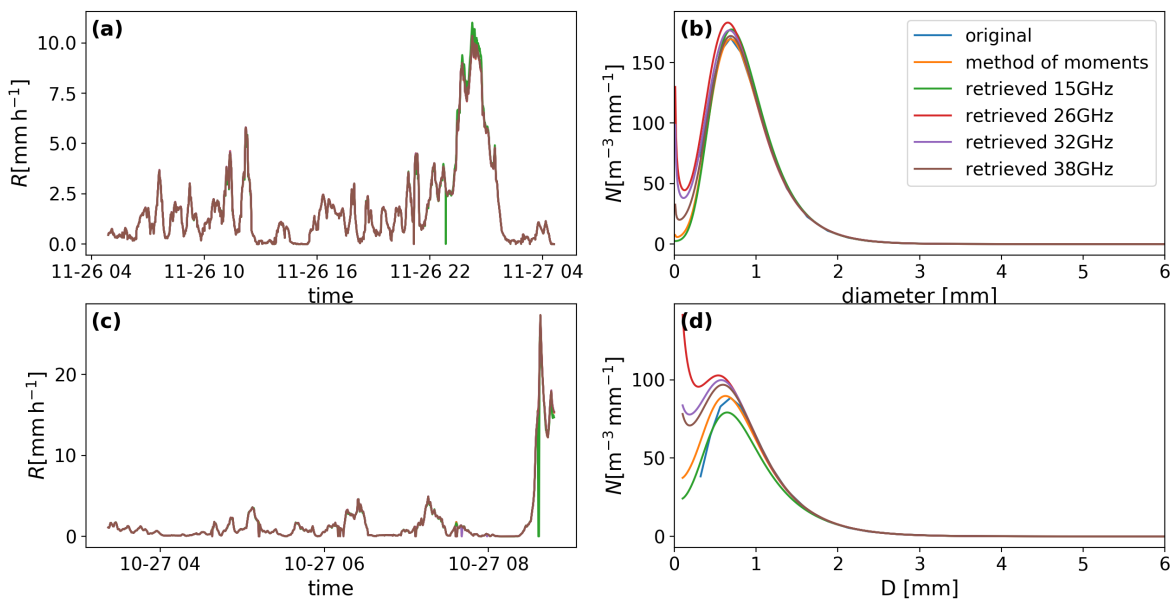

Figure 4.9: Rainfall intensity and mean drop size distribution retrieved on the basis of the two simulated events from the Ardèche region on 26/27 November 2012 (a) and 27 October 2013 (b). The retrievals are performed using the three-parameter method. The actual drop size distribution of the simulations and the DSD retrieved using the TS96 method is provided for comparison.

retrieval not only corresponds closely with the analytical method-of-moments approach, but also with the rainfall intensity derived from the original DSD. There are only a handful of outliers here, the exact timing of which is dependent on the carrier frequencies for which the retrieval is attempted. The MOR, MAD and 95AD of the rain intensity is given in Table 4.1 for retrievals based on several different carrier frequencies. Another way of assessing the performance of the retrievals is to visualize the temporal mean of the DSD. This is shown in Fig. 4.9b and d. We can see that the retrieval gives an overestimation for small diameters (with the exception of very low carrier frequencies), while becoming more accurate at larger diameters. At diameters larger than $1 \mathrm{~mm}$ the residuals are lower then $0.1 \mathrm{~m}^{-3} \mathrm{~mm}^{-1}$.

\subsection{Retrieval from disdrometers}

We apply both the two-parameter and three-parameter retrieval algorithms to the Wageningen disdrometer dataset. The results are shown in Fig. 4.10. We selected a single rain event on 27 July 2015 starting at 9:40 UTC and ending at 12:00 UTC. The first notable difference between the Ardèche and the Wageningen datasets is that in the latter the values for $\mu$ and $\Lambda$ are generally much higher. For both the TS96 approach and the numerical retrieval, values higher than 20 occur regularly for both the $\mu$ and the $\Lambda$ parameter, which is not consistent with what is typically found in the literature (e.g. Raupach and Berne (2016) for the Ardèche, Zhang et al. (2003) for Florida and Atlas and Ulbrich 
Table 4.1: Statistics of rainfall intensity $R$ relevant to the accuracy and precision of the threeparameter retrieval for two simulated events from the Ardèche region. All statistics are normalized with respect to the median of the original rainfall intensity.

\begin{tabular}{lcccc}
\hline \multicolumn{1}{c}{$15 \mathrm{CHz}$} & $26 \mathrm{GHz}$ & $32 \mathrm{CHz}$ & $38 \mathrm{GHz}$ \\
\hline \multicolumn{5}{l}{ Event 1 (mean: 1.77 $\mathrm{mm} \mathrm{h}^{-1}$, median: $1.08 \mathrm{~mm} \mathrm{~h}^{-1}$ ) } \\
\hline MOR & 0.0048 & 0.0084 & 0.0068 & 0.0049 \\
MAD & 0.0037 & 0.0046 & 0.0042 & 0.0035 \\
95AD & 0.355 & 0.0202 & 0.0298 & 0.0362 \\
\hline \multicolumn{5}{l}{ Event 2 (mean: 1.58 $\mathrm{mm} \mathrm{h}^{-1}$, median: $0.68 \mathrm{~mm} \mathrm{~h}^{-1}$ ) } \\
\hline MOR & 0.0017 & 0.0028 & 0.0045 & 0.0044 \\
MAD & 0.0065 & 0.0040 & 0.0037 & 0.0036 \\
95AD & 0.2304 & 0.0036 & 0.1197 & 0.0886 \\
\hline
\end{tabular}

Table 4.2: Statistics of rainfall intensity $R$ relevant to the accuracy and precision of the retrieval for 1 event and the whole dataset using the two parameter method based on the Wageningen disdrometer dataset. All statistics are normalized with respect to the median of the original measured rainfall intensity.

\begin{tabular}{lcccc}
\hline \multicolumn{1}{c}{$15 \mathrm{CHz}$} & $26 \mathrm{CHz}$ & $32 \mathrm{GHz}$ & $38 \mathrm{CHz}$ \\
\hline \multicolumn{5}{l}{ Event 1 (mean: $7.81 \mathrm{~mm} \mathrm{~h}^{-1}$, median: $5.46 \mathrm{~mm} \mathrm{~h}^{-1}$ ) } \\
\hline MOR & 0.0000 & 0.0084 & 0.0081 & 0.0083 \\
MAD & 0.0176 & 0.0251 & 0.0220 & 0.0241 \\
95AD & 0.2533 & 0.1603 & 0.1486 & 0.2202 \\
\hline 9 months (mean: 1.43 $\mathrm{mm} \mathrm{h}^{-1}$, & median: 0.69 & $\mathrm{~mm} \mathrm{~h}^{-1}$ ) \\
\hline MOR & 0.0003 & 0.0003 & 0.0001 & 0.0001 \\
MAD & 0.0103 & 0.0167 & 0.0151 & 0.0141 \\
95AD & 0.3389 & 0.2523 & 0.2328 & 0.2473 \\
\hline
\end{tabular}

(2006) for Kapingamarangi Atoll (western tropical Pacific)). We can also see that in several timesteps the $\mu$ and $\Lambda$ parameters in the retrieval are several times higher than they are in the TS96 method, but that this does not result in a significantly different rain intensity. Furthermore, when averaged over an entire event, the retrieval overestimates the number of drops with a diameter lower than $1 \mathrm{~mm}$ and underestimates the number of drops with a diameter between 1 and $7.5 \mathrm{~mm}$ when compared to the measured DSD (except for very low carrier frequencies). The correlation between the method-ofmoments derived $\mu$ and $\Lambda$ parameters is very high at $\rho=0.96$. The MOR, MAD and 95AD are given in Table 4.2. In general, the precision of the retrieval is an order of magnitude lower (higher MAD and 95AD), while the accuracy is actually higher (lower MOR) when compared with the Ardèche dataset. 

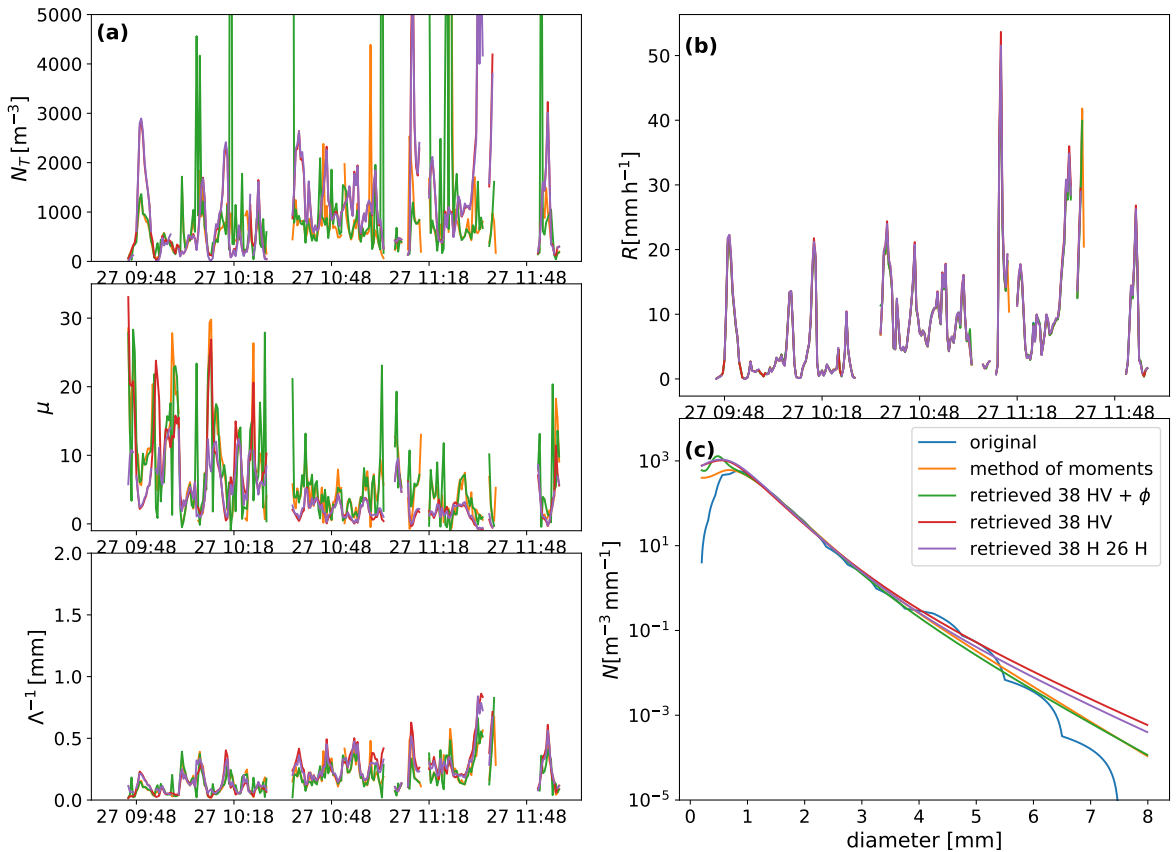

Figure 4.10: Retrieval of the event of 27July 2015 using only the two attenuations at $38 \mathrm{CHz}$ and a predetermined $\mu-\Lambda$ relation.

\subsubsection{Differences between two or three parameter retrievals}

We summarize the differences in accuracy and precision between the two-parameter and the three-parameter method in Table 4.3. The analyses are all performed with respect to a $38-\mathrm{CHz}$ dual-polarization retrieval (the third microwave link variable is the differential propagation phase). In addition, the number of failed retrievals (no solution at all) was $1.7 \%$ higher when using only 2 moments. This indicates that there is at least some advantage to using 3 moments.

However, the differences in accuracy and precision of the retrieval between the threeparameter and two-parameter retrieval as measured by a range of integer moments is small and in many cases the two-parameter retrieval proved to be more reliable. Especially the number of sub-millimeter rain drops is severely overestimated by using the three-parameter method, as shown in Fig. 4.11. Therefore, the addition of a third microwave link variable does not improve the retrieval (in many cases it harms the retrieval) and is unnecessary. Aside from needing one less attenuation moment, the two-parameter 


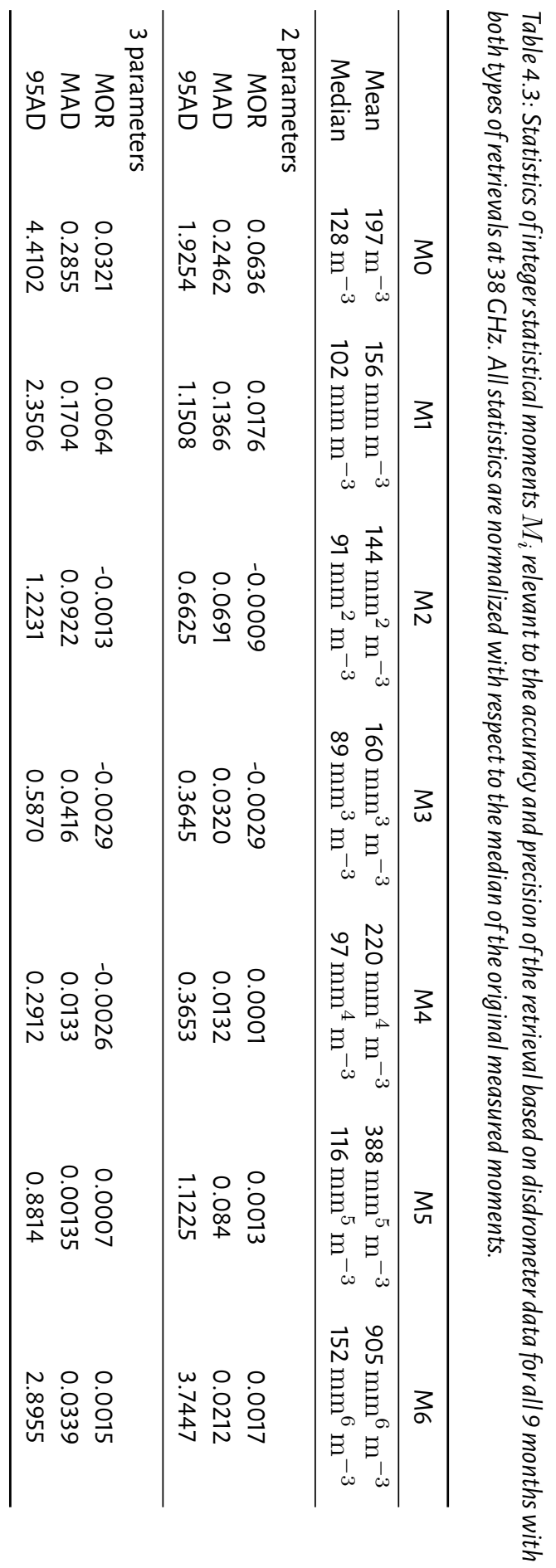




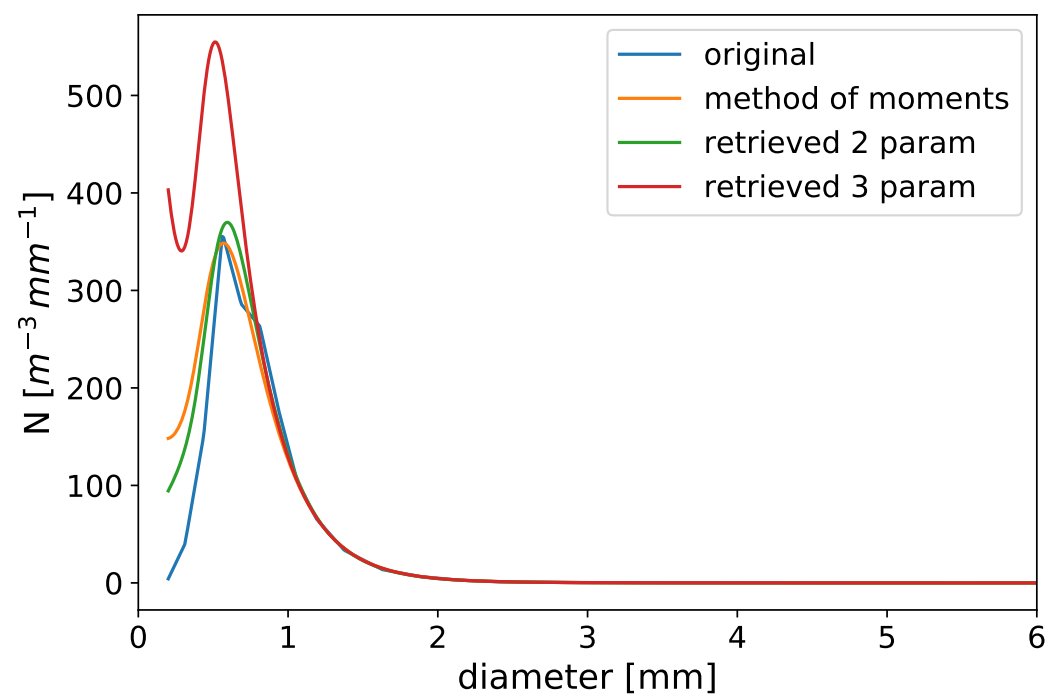

Figure 4.11: Mean over 9 months of DSD retrievals from simulated microwave link data based on disdrometer data from the Wageningen experiment using three methods: the analytical method of TS96, a numerical approach based on horizontal and vertical attenuation at $38 \mathrm{CHz}$ (twoparameter retrieval) and a numerical approach including both attenuations and the phase difference at $38 \mathrm{CHz}$ (three-parameter retrieval).

retrieval is also orders of magnitude faster. This is because the numerical part is univariate and therefore the dimensionality of the problem is reduced and also more efficient root finding methods other than gradient-based methods can be used (such as Brent's method). We do not need the workaround for local minima either, which is computationally very inefficient.

Because these results show that a three-parameter retrieval provides little added value above a two-parameter retrieval and because the two-parameter retrievals are far less computationally intensive than three-parameter retrievals we will restrict ourselves to two-parameter retrievals in the remainder of this chapter.

\subsubsection{Dependence on link frequency}

In order to determine the effect of the carrier frequencies of the links on the accuracy and precision of the retrieval we perform DSD retrievals at many different frequencies and calculate MOR, MAD and 95AD from the third order moment. We consider both dual polarization retrievals with frequencies ranging from $10 \mathrm{CHz}$ to $45 \mathrm{CHz}$ (with steps of $1 \mathrm{CHz}$ ) as well as dual frequency retrievals using every combination between 10 and $45 \mathrm{CHz}$. This range contains the bulk of microwave link frequencies found in typical communication 

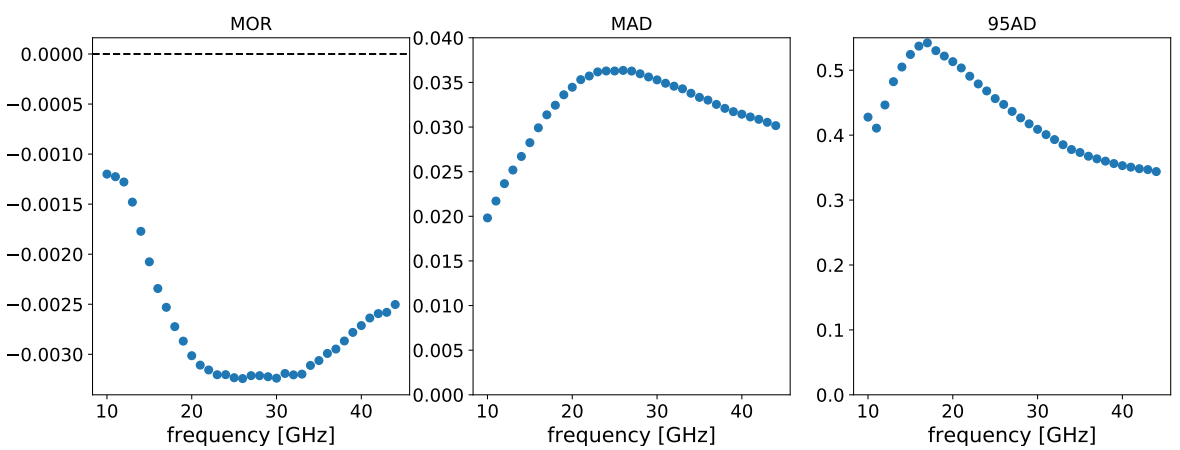

Figure 4.12: MOR, MAD and 95AD of the third order moment of the DSD estimated using a twomoment dual-pol retrieval as a function of carrier frequency based on disdrometer data. All statistics are normalized with respect to the median of the moment of the original measured DSD.
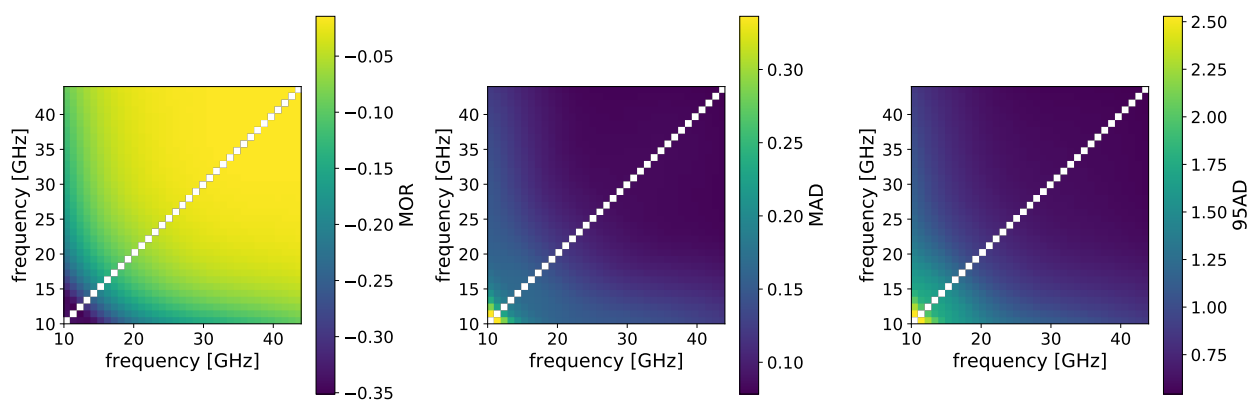

Figure 4.13: MOR, MAD and 95AD of the third order moment of the DSD estimated using a twomoment dual-frequency retrieval as a function of the two carrier frequencies based on disdrometer data. All statistics are normalized with respect to the median of the moment of the original measured DSD.

networks. The results are shown for dual polarization in Fig. 4.12 and for dual frequency in Fig. 4.13. We can see in Fig. 4.12 that the accuracy and precision is high for all frequencies. However, the accuracy is pessimal for frequencies between 22 and $34 \mathrm{CHz}$. Similarly, MAD is largest around $25 \mathrm{CHz}$ and 95AD is largest around $17 \mathrm{CHz}$. Therefore, for an optimal retrieval those intermediate frequencies should be avoided. Fig. 4.13 shows that the accuracy and precision of dual frequency retrievals is highest when both frequencies are high. The difference between the two frequencies does not seem to matter much; when the two frequencies are far apart the precision and frequency are actually slightly lower. Predictably, there are no solutions found when the frequencies are exactly the same. It should be noted that for this simulation, the effect of noise is not taken into account. It is expected that this would influence the retrieval the most when the frequencies are close together. 

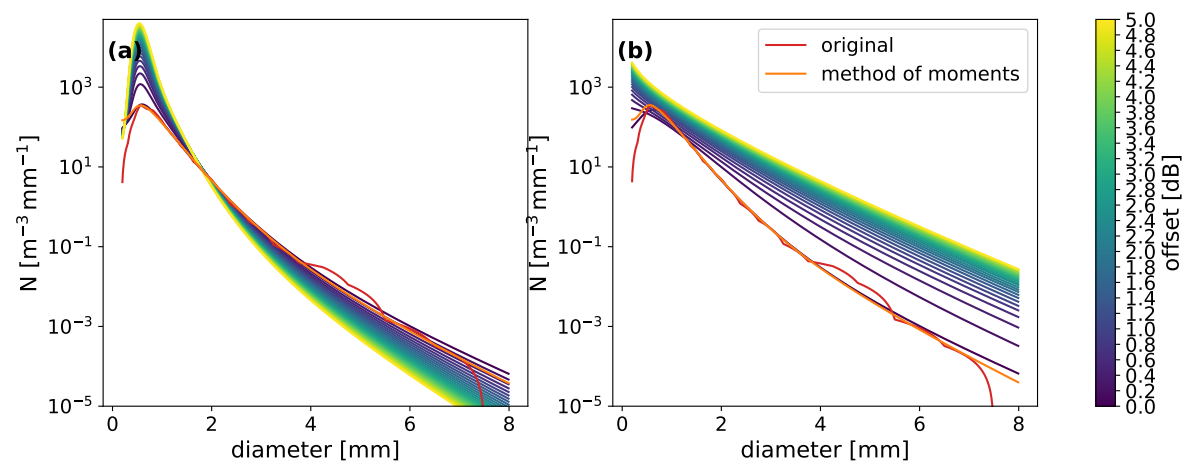

Figure 4.14: DSDs of the two-moment retrieval $(38 \mathrm{CHz})$ of the 9-month Wageningen disdrometer dataset with an offset in the attenuations of $\mathrm{O}-5 \mathrm{~dB}$.

\subsubsection{Sensitivity to attenuation bias}

Because our retrieval algorithm uses ratios of attenuations as input, it is important that a reliable baseline power level is established from which to calculate the attenuations. To assess the sensitivity of the retrieval technique to inaccuracies in the baseline (dry) power level, we perform the two-moment retrievals based on the simulated attenuations from the disdrometer measurements but with an offset added to all input attenuations. Figure 4.14 shows the resulting DSDs averaged over 9 months for attenuation offsets between 0 and $5 \mathrm{~dB}$ and a path length of $2.2 \mathrm{~km}$. For this analysis we chose two combinations of links that we will also later use for the actual link measurements: a dual polarization retrieval at $38 \mathrm{CHz}$ and a dual frequency retrieval at $26 \mathrm{CHz}$ and $38 \mathrm{CHz}$. We can see in Fig. 4.14a that the addition of an offset to the attenuation leads to an overestimation below $2 \mathrm{~mm}$ and an underestimation above $2 \mathrm{~mm}$ for the dual polarization retrieval. The introduction of an offset has no effect at $2 \mathrm{~mm}$. the effects are largest below $1 \mathrm{~mm}$. The effects for a dual frequency retrieval are quite different, as can be seen in Fig. 4.14b. There is an overestimation at all diameters. The overestimation is smallest around $1 \mathrm{~mm}$ and increasing towards higher and lower diameters. Overall, the mean bias is larger than for dual polarization retrievals and the shape of the DSD is especially sensitive to small offsets in the base power level.

\subsection{Experimental link retrieval}

Using the double-moment retrieval method we estimated the DSDs from actual link measurements of the Wageningen setup. The baseline power level of the links showed considerable fluctuations over the course of the measurement period. Therefore, it was not feasible to perform retrievals for the entire 9 month dataset. We selected the event of 27 July 2015 (see Fig. 4.15) because the power levels of the links in the period surrounding this event showed relatively little fluctuations. We determined a suitable constant baseline 

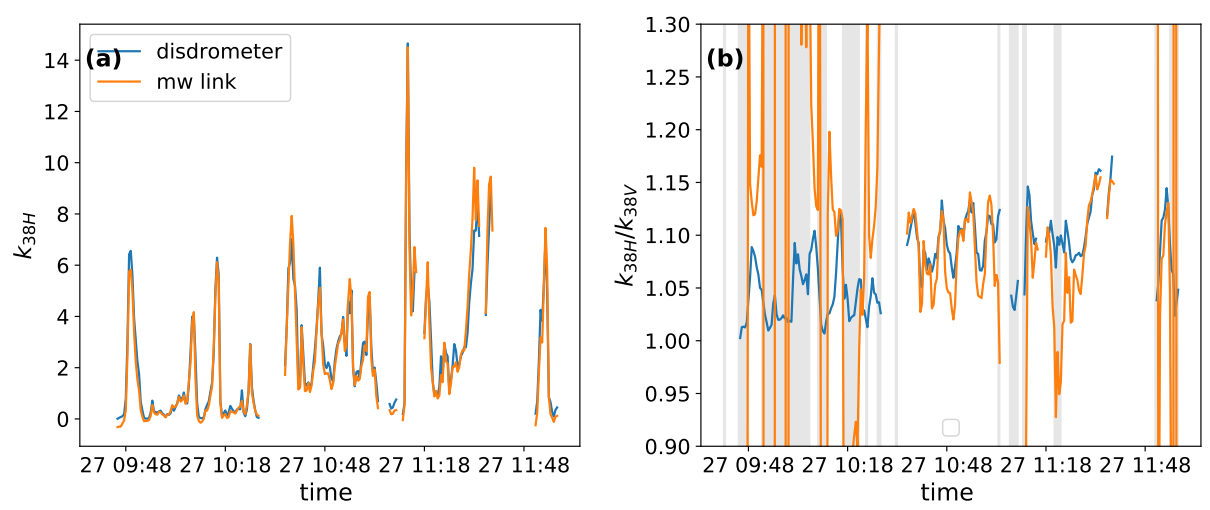

Figure 4.15: a) Link attenuation at $38 \mathrm{CHz}$ with vertical polarization. Time series are shown for link measurements (orange) as well as attenuation computed from disdrometer data (blue) for the event of 27 July 2015. b) The ratio of the attenuations of the horizontally and vertically polarized signal. Shaded areas indicate intervals where the ratio is outside of the solvable range for the dual polarization retrieval (1.00-1.25).

power level calibrated for this event. The measured attenuation after subtracting a baseline for this event is shown in Fig. 4.15 and compared with the path-average attenuation derived from the disdrometer measurements; The retrieval results are given in Fig. 4.16.

The resulting DSD is very similar in shape to that obtained in the simulations, with overestimations especially at smaller diameters but with the general shape of the DSD preserved. Closer inspection reveals that the bias and scatter compared to the original DSD are actually up to two orders of magnitude higher than in the simulations, as can be seen in Table 4.4. Nevertheless, at the important higher order moments related to e.g. liquid water content, rain rate, kinetic energy and radar reflectivity the bias is around $7 \%$ for the dual polarization retrieval. The scatter as indicated by the MAD is about one order of magnitude higher than the simulations across all moments. However, when taking the 95AD as measure of scatter, the order of magnitude is the same. There are a few more intervals with no solution at all when compared to the simulations. These correspond with ratios of observables that are outside the range of the forward model (see Fig. 4.6). The ratios for the dual polarization retrieval are illustrated in Fig. 4.15b. These intervals with extremely low or high ratios between attenuations mostly (but not always) occur when the rain intensity is low and thus other sources of signal variability are more dominant. Overall the dual polarization and dual frequency retrievals have a similar performance in this case. However, using two frequencies instead of two polarizations leads to a higher accuracy for low order moments (up to 4th), but lower accuracy for higher order moments. No plausible solutions for the retrieval where obtained when using the phase difference instead of one of the attenuations or with a triple variable retrieval. Very few intervals led to convergence at all with this configuration. 


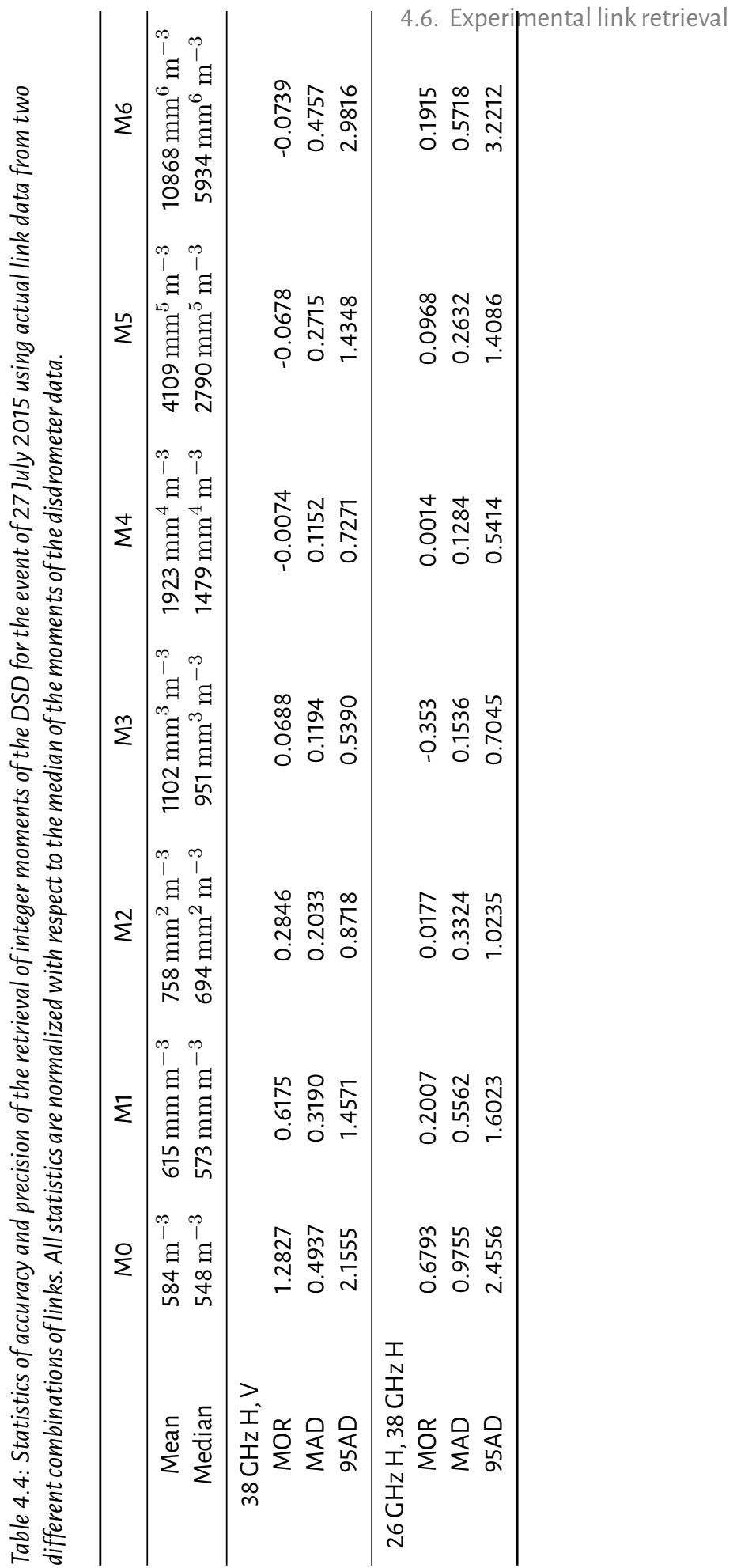



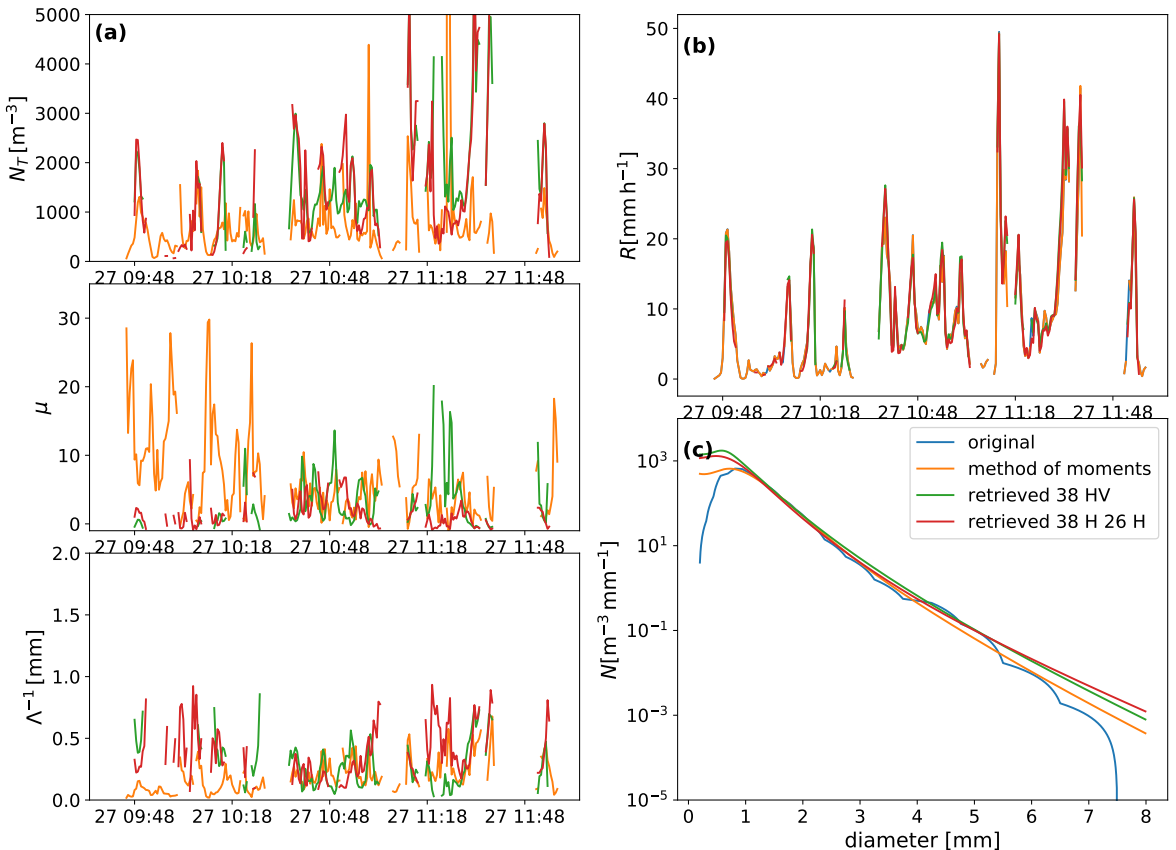

Figure 4.16: Retrieval results for the event of 27July as measured by the $38 \mathrm{CHz}$ instrument using horizontal and vertical polarization and the $26 \mathrm{CHz}$ instrument using horizontal polarization. The method of moments results are derived from the five disdrometers for the same event.

\subsection{Discussion}

\subsubsection{Feasibility in practice}

Constraints on the feasibility of this method in practice fall into three broad categories: availability of multiple link signals on the same path; quality of the available signals; and real-time processing speed.

The use of a three-moment retrieval means that three moments on the same path need to be available. This is rare in commercial networks; Therefore this method is most readily applicable to dedicated research networks. There are several different combinations of moments to choose from. However, in our approach we focused on the combination of a horizontal attenuation, vertical attenuation and phase difference at the same carrier frequency. This allows the use of a single set of antennae for all three moments, allowing for the use of a more compact and cheaper device (such as the device that was used in 
this test setup).

The second concern is with regard to the quality and reliability of the signal. In order to apply the method in practice it is essential that a baseline (no rain) signal is accurately determined. because the method relies on the ratios of attenuations, small deviations in the baseline determination can result in large deviations in the retrieval (and even nonconvergence). No such problem exists in principle with regard to the phase difference; It is independent of any baseline as long as that baseline is indifferent to polarization. However, phase difference on its own is not sufficient for the retrieval. To have a chance at a successful retrieval the baseline needs to be as invariant as possible. Where it is not, the variability should be accurately modeled and predicted from auxiliary measurements. In our own preliminary attempts we found our instruments lacking in stability. In particular the clinging of drops to the antenna cover (as described in chapter 2) seems an intractable problem. However, as was also described in that chapter, we found that a former commercial microwave link had a much stabler baseline and furthermore the effect of wet antennas was much more manageable for that particular device. This provides a hopeful perspective for the application of this method to commercial networks.

The third constraint is only relevant when real-time processing is required. The threemoment method is relatively wasteful with computing cycles because of the repeated reinitialization of the root finding process. We might expect this to become a bottleneck. However, in its current implementation the processing of 9 months of data from one link requires roughly three hours of wall time on a high-end desktop workstation. This is while utilizing $10 \mathrm{CPU}$ cores simultaneously. Such a setup is therefore expected to be able to process a network of more than 2000 links in real-time. Nevertheless, reducing this computational load would make real-time retrievals more feasible on low-end (embedded) hardware as well. We already found that the specific programmatic implementation of the root-finding algorithm can make an order of magnitude difference in computation time. The use of pre-computed lookup tables may help to bring down computation time in a real-time setting. The two-moment method requires far less computation (e.g. processing the same 9 months of data with the same workstation requires only 5 minutes), which makes it far more suitable for real-time processing.

\subsubsection{Caveats}

There are a number of caveats to our methods which could influence the interpretation of the results: Firstly, we use a threshold of 50 drops per disdrometer to filter out low quality measurements before calculating the mask or $\mu-\Lambda$ fit. How high this number should be is debatable (e.g. Uijlenhoet et al. (2006)). A threshold that is too low might allow for many erroneous measurements that are not representative of rain. On the other hand, a threshold that is too high might result in too many reasonable measurements being rejected. This might mean the results are less statistically representative and possibly biased towards high rain intensities. The number used here is therefore a compromise and the resulting relationships do change somewhat depending on the threshold chosen. A similar consideration applies to the choice too filter on a per instrument basis in- 
stead of on the basis of the total drop count. Filtering on a per instrument basis makes it more likely that all instruments where measuring correctly. However, it does bias the measurements towards more homogeneous rainfall. Considering the small spatial scale of the measurements we considered and the high spatial correlations therein this an acceptable loss.

Another consideration is the use of the mask itself. The mask is determined on the basis of the measured disdrometer data. We then use this mask in, among others, the retrieval of the DSD from the disdrometer-derived simulated variables. There is certainly something circular about this. Nevertheless, this data is never used as input for the rootfinding procedure itself. It is only used a posteriori to assess whether the results fall into a plausible range of values. Potentially more serious is the use of the predetermined $\mu$ $\Lambda$ relationship in the case of the two-parameter retrieval. In this case, the relationship determined on the basis of the disdrometer measurements is used more directly in the retrieval. This might have colored the results somewhat in favor of the two-parameter retrieval as opposed to the three-parameter retrieval. Somewhat alleviating is the fact that both the $\mu-\Lambda$ relationship and the mask are determined from the total of all 9 months of disdrometer measurements, not from the specific event in question.

The third consideration is the underlying assumption that the untruncated gamma distribution is applicable throughout the diameter domain. It is on the basis of this assumption that we treat the values of $\mu$ and $\Lambda$ derived from the TS96 method of moments as the correct parameter values. The gamma distribution has a non-zero value up to positive infinity. Meanwhile, there are both physical and instrumental cutoffs to the maximum drop size that can occur. This would suggest that a truncation should be included in the expression of the gamma distribution. However, the truncation of the gamma distribution at high diameters is not relevant in this case because we can see in Figs. 4.7a, $4.9 \mathrm{~b}$ and $\mathrm{d}, 4.10 \mathrm{c}, 4.11$ and $4.16 \mathrm{c}$ that the gamma distribution corresponding to the DSDs under consideration tends to zero (or $<10^{-2} \mathrm{~m}^{-3} \mathrm{~mm}^{-1}$ ) before the instrumental cutoff. We can also observe from those figures that the systematic deviation between the measured (interpolated) DSD and the DSD obtained from the method of moments is small. Instrumental cutoff at the small end of the diameter scale is relevant in this case, but the effects of this on higher order moments is minute. Regardless, since the attenuation/phase based retrieval in this case is limited to three parameters, it is not possible to include a cutoff there. A retrieval using even more signals might make this possible, but this would limit the practical applicability of this method further.

\subsection{Conclusions and outlook}

Using both simulations and actual link data we have shown that a DSD retrieval on the basis of multiple microwave link variables can be successful and highly accurate. Both the use of dual-polarization and dual-frequency retrievals is feasible. However, the use of dual-polarization is less sensitive to inaccuracies in the base power level than the use of dual-frequency links. Simulated retrievals using a variety of frequencies show that, at 
least between $10 \mathrm{GHz}$ and $45 \mathrm{CHz}$, the accuracy and precision of the retrievals is very high for all frequency combinations. Therefore, the frequency chosen for a dual polarization retrieval is not very important. Nevertheless, when a choice is available, our simulations indicate that frequencies at the lowest end or the highest end of the range are preferred. Furthermore, at the lowest end of the frequency range there is less attenuation in general and therefore a smaller difference in attenuation. This could more easily be obscured by noise or quantization effect. Therefore, the higher end of the tested frequencies are optimal. For dual frequency retrievals, bias and random error are an order of magnitude higher than for the dual polarization retrievals. If a dual frequency retrieval is attempted, both frequencies should be as high as possible to minimize the bias and random error. Curiously, in our simulation, retrievals performed using two frequencies 1 or $2 \mathrm{GHz}$ apart were just as accurate as retrievals using two frequencies that are further apart. However, it is expected that this would change if noise is taken into account.

In our field experiment we tested a dual-frequency retrieval using $26 \mathrm{GHz}$ and $38 \mathrm{CHz}$ as well as a dual polarization retrieval at $38 \mathrm{GHz}$. Both retrievals produced good results for a selected summer event where other attenuating atmospheric phenomena where not present. The feasibility of the retrieval does however depend very much on a stable base power level, which was not guaranteed in our experiment. The Nokia link (former commercial link) is promising in this respect, because it was much stabler and less sensitive to e.g. temperature fluctuations and antenna wetting.

Using phase differences in addition to attenuations is feasible in the simulations. However, in practice these measurements are not accurate enough to yield meaningful solutions. In most instances no convergence was obtained. We have also shown that using three microwave link variables yields no improvements over a retrieval using only two variables, which is also computationally faster and more readily applicable in operational settings. At least in comparable climatologies to those treated here, a predetermined $\mu$ $\Lambda$ relation suffices to determine the gamma DSD parameters from two attenuations.

A follow up experiment using different microwave links of similar frequencies (preferably commercially available ones) is needed to determine if the base power level of commercial links is sufficiently stable for reliable continuous observations. A tally should also be done on the number of dual polarized links in cellular communications networks to determine if it is feasible to retrieve spatial DSD information from such networks or whether this technique is only applicable to some individual link paths, either from commercial or research networks. Another concern is the quantization of data from commercial link networks. As the difference between the attenuation of the horizontal signal and the vertical signal is often a fraction of a $\mathrm{dB}$ and data available from such networks are often rounded to $0.1,0.5$ or $1 \mathrm{~dB}$, this might limit the applicability of this method. Therefore, the possibility of collecting higher resolution data from such instruments should be looked into. 


\section{Chapter 5}

\section{Synthesis}

\subsection{Summary and conclusions}

In this thesis data from a unique field experiment was analyzed to gain more insight into the error sources in microwave link rainfall estimation, the spatio-temporal variability of rain at a typical link scale, and the possibility for retrieving raindrop size distributions from microwave links.

In Chapter 2 of this thesis we first tested a naïve retrieval algorithm applied to the microwave link measurements on the basis of a power-law relationship and compared the results with five disdrometers positioned along the path. In this naive algorithm we do not take into account any error sources and thus assume any deviation in the received signal level to be attenuation by rain. We then used a simple power law relationship derived from the disdrometer measurements to calculate rainfall intensity. This approach results in good correlations for high rain intensities but a strong overestimation as well. Correlations become weaker at low rainfall intensities. There is a significant difference in the amount of overestimation between the different link types. In particular, the former operational link (Nokia) experienced a much less severe overestimation than the dedicated research links we used. This analysis provides a baseline accuracy that can be used to assess the effectiveness of improved algorithms. To get any more accuracy requires a more detailed accounting of alternative sources of apparent attenuation.

One of the major sources of error in retrieving rainfall intensity from microwave links is the fact that the antennas themselves become wet. This is sometimes accounted for in retrieval algorithms as a constant extra attenuation during periods of rainfall (e.g. RAINLINK (Overeem et al., 2016a)). In Chapter 2 it was shown that the wetting of antennas during rainfall can produce a significant attenuation that is in the order of magnitude of the attenuation due to rainfall itself. More problematically, depending on the type of antenna cover, this attenuation is not necessarily constant in time and in fact can have a random element to it due to the beading behavior of water on the semi-hydrophobic material of some of the antenna covers. The Nokia link was not affected by this, because of 
the different material characteristics of its antenna cover. It is still an open question how many antenna types found in operational settings are affected by this phenomenon. This effect can lead to severe over-or underestimation when the extra attenuation is assumed constant in the retrieval. Moreover, wetting of antennas does not only take place during rain, but also during fog and in the early morning due to dew. However, this thesis does not provide conclusive evidence regarding the relative effect of fog droplets in the atmosphere along the link path and the wetting of the antennas due to the presence of those drops.

The received signal level of the microwave links in the Wageningen experiment also showed significant low-frequency variability over the course of the experiment (especially the dedicated research links) even when the antennas were completely dry. These fluctuations in received signal level were highly correlated to ambient temperature fluctuations. It was found that this temperature dependence can be as high as $-0.2 \mathrm{~dB} \mathrm{~K}^{-1}$, which is much larger than the expected variation due to temperature-dependent absorption by atmospheric constituents. Just like with the wetting of the antennas, the magnitude of this phenomenon is dependent on the specific link hardware used with the Nokia links having a signal level dependence on temperature that is an order of magnitude lower.

The third major source of error found in this study was temporary obstruction by large metallic objects. The locations of our instruments were carefully chosen so as to be unobstructed and reasonably far removed from possible obstructions near ground level. The transmitters and receivers were both placed on the tallest buildings in their direct surroundings and under the path between them only low-rise housing was present. Nevertheless, unforeseen circumstances such as construction works brought large metallic objects (e.g. a construction crane) exactly into the link path on several occasions. If we had not installed time-lapse cameras on our instruments we never would have known this. These obstructions caused very sudden, sharp drops in the overall signal level. Although mobile communication link locations are similarly selected to be unlikely to be obstructed, the fact that these links are often installed in urban areas and the fact that these occurrences are difficult to avoid or predict when planning a network makes me believe that such occurrences may still be quite frequent. Therefore, it is worth the effort to devise a method to detect or compensate for these disturbances.

In the Wageningen experiment two links operating at the same frequency and polarization were used along the same path. In theory such links should produce the same results. However, as was shown in Chapter 2, large differences in the signals of both links existed. These differences are for the most part related to either wetting of antennas or to temperature sensitivity of the hardware. This presents a challenge in two ways: It increases the complexity of the compensation mechanisms; and it may necessitate devicespecific corrections, which is challenging when retrieving from an entire network that consists of different types of links.

In Chapter 3 we investigated the spatio-temporal correlation structure of the rainfall field in our experiment based on the disdrometers we had available. We extended this analy- 
sis to one disdrometer network in Rotterdam and one gauge network across the Netherlands, to see if we could find a consistent parameterization. We found that a single modified exponential decorrelation function is able to describe the spatial variability across a broad range of scales, from smaller than a single link $(<1 \mathrm{~km})$ to the scale of the entire Dutch link network ( $\sim 300 \mathrm{~km}$ ). The knowledge gained in this chapter could be used to quantify the representativeness error induced by rain gauges versus microwave links.

In Chapter 4 a technique for retrieving drop size distributions from an array of collocated microwave links was developed and analyzed using data from the disdrometers placed in Wageningen as well as DSD fields based on data collected in France. DSD retrievals can be performed using two links with different carrier frequencies or two links with different polarizations. It was shown that both kinds of DSD retrieval using two link variables are feasible. It was also shown on the basis of these simulations that a dual-polarization link combination is preferable to a dual-frequency link combination in terms of accuracy. This is fortunate considering that dual-polarization links are more easily set up than dualfrequency links and therefore are probably more common in telecommunication networks. This is because dual polarization links can be realized with a single set of antennas, while dual frequency links would need a double set of antennas if the difference between the frequencies is significant. Additionally, optimal carrier frequencies are usually chosen based on the length of the link and are thus mostly similar over the same path. Simulations based on the disdrometer data showed as well that dual-polarization retrievals based on carrier frequencies of around 35 to $45 \mathrm{GHz}$ are slightly more accurate than those based on retrievals at lower frequencies. Finally, retrievals using three link variables were also attempted. It was shown that such retrievals offer no added value over two-variable retrievals and require significantly more computational expense. I therefore conclude that, going forward, research focus should be on dual-polarization DSD retrievals.

The final part of this thesis dealt with the retrieval of DSDs from the actual links of the Wageningen experiment using the techniques developed and analyzed in the previous part. The retrieval was performed on a selected event where very little influences on the attenuation aside from rain itself were present. The results prove the feasibility of the technique in practice as long as the baseline power level is stable. However, a fundamental problem is that this baseline is highly variable in the dedicated research links used in this experiment, as was explored in Chapter 2. Especially the DSD retrieval, which is dependent on the ratios of attenuations, is sensitive to such offsets. This was also demonstrated by adding an artificial offset to the simulations in Chapter 4. Especially during low rainfall intensities, the offset can even push the ratio outside the solvable range. Because of the random attenuation due to wet antennas and the temperature-related fluctuations, the ratios in most events were too distorted to obtain a solution. This demonstrates the importance of correcting for these influences first or to use links that are less sensitive to these in the first place. As was seen in Chapter 2, the Nokia link has a much stabler baseline than our research links, but since only one was available in the experiment, it was not possible to test the DSD retrieval algorithm on this device. The very stable performance of this link, however, makes me hopeful that this technique can be successfully applied to similar commercial links, provided the quantization is fine-grained enough. 
A common theme in all these explorations is the variation in response between ostensibly similar instrumentation. This was an unexpected result and in many ways hampered a more in-depth analysis of the phenomena under study within the time available. All in all the commercial link transceivers that were investigated were less prone to error due to wet antennas or temperature variations than the dedicated research links.

\subsection{Recommendations for future research}

The investigations started in the course of this research still leave many questions unanswered. In fact, while Chapter 2 provided a comprehensive overview of environmental and instrumental influences affecting the quality of the retrieval of rainfall, there was little room left for an in-depth investigation into possible solutions to each of these problems. It is therefore my hope to have the opportunity to investigate some of these further.

In my view, investigations into the possibility for snowfall retrieval, while scientifically interesting (and challenging), should not have a high priority for future research in the Netherlands. During our investigation in the Netherlands, there where only a handful of short snowfall events in 1.5 years. This is too little for a robust analysis. Furthermore, areas of the world where microwave link measurements have the biggest potential are mostly tropical and subtropical and thus snowfall retrieval would be even less relevant there. Mountainous areas are another important application area for microwave link environmental monitoring and in those areas snowfall is certainly relevant. However, the very weak attenuation by snow (e.g. Matrosov, 2007) would make this extremely challenging. Wet snow, in contrast, can cause a very strong attenuation (Paulson and Al-Mreri, 2011), as was also seen in this thesis, which only increases the challenge in quantitatively relating attenuation to snowfall intensity. There is some evidence that multiple collocated links can provide a solution here (Ostrometzky et al., 2015), which could be investigated further.

Wet antennas and the myriad of phenomena associated with them are perhaps the area where the most significant gains can be made. A number of different techniques for separating dry and wet spells have already been investigated (Overeem et al., 2013; Schleiss and Berne, 2010; Chwala et al., 2012; Wang et al., 2012; Rahimi et al., 2003), but none of them aim at separating different attenuation sources that can occur concurrently. I believe an attempt to do this would be worthwhile.

On the other hand, the most severe problems that we found with regard to wet antennas, may only be applicable to a handful of antenna types. Focusing our attention on these specific anomalies could be a mistake, in my opinion. We should be careful to not study our own instruments instead of the phenomenon. The same could be said for the likely temperature-related oscillations. These where noticeable in all the links under study, but the magnitude of the oscillations was very dependent on the specific link instrument.

In order for further research to be more effective, I suggest that a more systematic assessment and publication of the capabilities of existing networks is essential. This would 


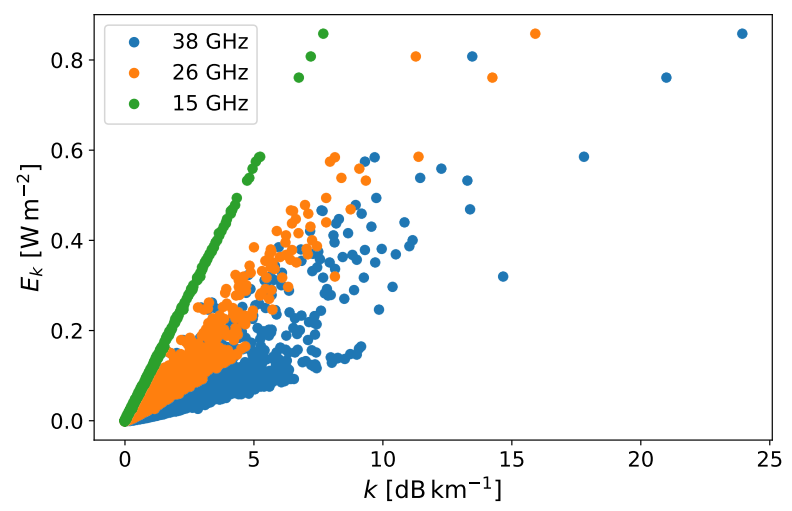

Figure 5.1: Kinetic energy flux as a function of specific attenuation at several different carrier frequencies. These are simulations based on the Wageningen disdrometer dataset.

include a global tally of link systems in use, with their operating frequencies and polarizations but also their manufacturers and models. This would mostly be an 'accounting' study, to collate existing information from different sources and make them consistent and readily available. This should also include relevant testing data that manufacturers of the link systems in question might have. Where that is not available or not adequate, a series of more controlled lab experiments should be conducted with some of the most commonly used hardware, specifically to test the response to the phenomena relevant to rainfall retrieval and atmospheric monitoring in general. Although this might seem like busywork, I believe it would help to focus further research efforts to where they can make the most difference and in the long run save more effort than it would cost.

Another interesting follow-up to the research in this thesis is with regards to our investigation of rainfall variability in Chapter 3 . I believe it would be interesting to investigate, in addition to the rain rates, the variability of DSDs as well. Unfortunately, although the instruments in Rotterdam were laser disdrometers, we did not have access to the DSDs measured by them. This limited the scope of the investigation. Networks of disdrometers have been used to investigate variability of DSDs at a small spatial scale (e.g. Jaffrain and Berne, 2012), but an investigation of DSD variability at a range of scales similar to the investigation in Chapter 3 would be quite novel. In the light of the promising results of Chapter 4 , the exciting possibility of investigating DSD variability using the operational microwave link network presents itself. If enough suitable links can be found, this would be a very worthwhile avenue for follow-up research.

In order to further test the possibilities for multi-link retrievals a new field experiment could be set up. This would be similar in configuration to the setup used for this thesis, but using only link devices used in actual commercial link networks. This would allow to ascertain the actual practicability of these retrievals better than could be done with the 
setup used for this thesis, which used specially constructed experimental links (which then turned out to be less stable than off-the-shelf devices).

An interesting further avenue of research is the possibility to use microwave links to retrieve proxies for soil erosion by rain. An important proxy is the kinetic energy of rain; This can be derived from the DSD and therefore the technique developed here for multi-link retrievals of DSD could be expanded to yield information on soil erosion. A preliminary investigation, illustrated in Fig. 5.1, reveals that especially at very low link frequencies a single link might suffice as well.

\subsection{Application perspectives}

In well-gauged areas in Europe, the added value of microwave link measurements is limited. In addition, in flat, densely populated countries such as the Netherlands the number of microwave links has been decreasing in recent years. This is due to them being replaced by fiberglass connections. In other parts of Europe, in particular mountainous areas, this is not the case, because fiberglass cables would be too costly to install compared to the potential benefit in signal reliability. In the future, a decrease in long-range backhaul links is expected in favor of fiberglass connections, while at the same time an increase in short-range high-frequency links is expected for last-mile hops in urban areas to accommodate increased bandwidth requirements (Ericsson, 2017). Nevertheless, these areas are among the first to actually have link data made available to the research community. The fact that these areas are already well-gauged makes them also very suitable for calibration and validation studies.

The work done in this thesis with regard to DSD retrievals does reveal a way in which microwave links can provide data that is not otherwise available in Europe. Polarimetric radar is only available in limited areas and furthermore radars will always measure at a significant height above the earth's surface, so that information from these radars will not necessarily correspond to what is falling at the ground. Therefore, collocated microwave links might be able to provide information on additional rainfall variables that radars cannot. At the moment $20 \%$ of all link hops use multiple link transceivers at different frequencies or polarizations and this number is rising globally to meet with increased bandwidth demands (Ericsson, 2017). This signifies that dual-frequency or dualpolarization links could be numerous enough for mapping purposes. Furthermore, the merging of radar and microwave link precipitation data can increase the overall quality of rainfall data products.

The potential for applications in other parts of the world, in particular Africa, South-America and large parts of Asia, is much greater. Building on the research described in this thesis and other related work, a practical demonstration is being prepared for operational microwave link retrievals in Sri Lanka. Other regions where the potential of microwave link rainfall estimation has been demonstrated include Brazil (Rios Gaona et al., 2018) and Burkina Faso (Doumounia et al., 2014). In order to provide even better rain- 
fall data products in these regions, merging microwave link and satellite data provide a promising prospect (Rios Caona et al., 2017). Because the kinetic energy of falling raindrops can be derived from the DSD, microwave links might even be used to monitor the potential for rain erosion in an area.

An ongoing practical concern potentially hindering the widespread use of microwave link derived rainfall measurements is the difficulty of obtaining the data from the various telecommunication providers and collating it into an end-user product in real time. This is not a fundamental technical challenge anymore, as e.g. Chwala et al. (2016) have proven. Neither is it necessarily an economic challenge, considering the negligible additional cost that such data collection would have above the normal operational cost of microwave links. Even if no profitable business model could be built upon this technique (a big if), that would be no reason to let this societally valuable information go to waste. The costs can easily be financed by governments or NCOs or even the businesses themselves in the context of societally responsible entrepreneurship. However, I do not believe it is necessarily a lack of interest on the parts of the telecommunication providers that is at this moment the biggest challenge either, considering the gracious contributions we and other researchers in this field have had from telecommunication providers in terms of access to network data and even equipment donations. The last hurdle that needs to be taken now is that of inertia and organization. In overcoming this, coordination on the level of international trade representative GSMA and UN regulatory agency ITU would be very welcome.

In order to facilitate the rapid adoption of these techniques, the publication and maintenance of practically usable software packages to easily deal with the collection and processing of microwave link data in a variety of settings with limited user input is needed. Packages such as RAINLINK (Overeem et al., 2016a) are an encouraging first step and the software I developed to facilitate the research reported in this thesis could be molded into an even more generalized package. In tandem, efforts should be made to standardize the data formats used by the various packages under development by multiple groups at the moment. This would serve greatly to facilitate intercomparison studies and, in the long run, network and border crossing assimilated data products.

As new $5 \mathrm{C}$ techniques are gradually being developed and rolled out, this will bring with it new opportunities for opportunistic sensing. 5C bandwidth requirements will necessitate the introduction of a new short-range high-frequency $(70-80 \mathrm{GHz})$ class of links in a dense network. The increased spatial density would make higher resolution rainfall mapping possible. These links would also mainly be placed in cities, which is also where the need for high-resolution real-time rainfall monitoring is greatest considering the fast response of urban areas to hydrometeorological input. Furthermore, the introduction of the 5C NR (New Radio) standard means that end-nodes such as smartphones will soon also operate in the frequency range between 20 and $45 \mathrm{GHz}$ (Ericsson, 2018), previously only used for backhaul links, which is optimal for rainfall measurement. This will open up a whole new range of possibilities for opportunistic environmental monitoring, but will also be much more technically challenging (as well as potentially posing privacy prob- 
lems). Recent research into the use of crowdsourced personal weather stations (De Vos et al., 2017) and smartphones as temperature sensors (Droste et al., 2017) shows the potential of using a large number of individually unreliable measurements to attain reasonably accurate results.

Because these techniques are currently under development, there is an opportunity for scientists in the remote sensing and hydrometeorological communities to have environmental monitoring applications taken into consideration from the get-go. Because organizational inertia is currently the main hurdle to widespread adoption, this opportunity must not be neglected. Therefore, two things should be done: Researchers in the field should keep and maintain contact with the telecommunication equipment manufacturers and their engineers as well as telecom operators to make their case; Secondly, preliminary research using simulations should already be conducted to assess the possibilities for retrieval of environmental quantities based on the expected characteristics of $5 \mathrm{C}$ equipment. 


\section{Bibliography}

Angulo-Martínez, M., and A. Barros (2015), Measurement uncertainty in rainfall kinetic energy and intensity relationships for soil erosion studies: An evaluation using PARSIVEL disdrometers in the southern Appalachian mountains, Ceomorphology, 228, 28-40, doi: 10.1016/j.geomorph.2014.07.036.

Atlas, D., and C. W. Ulbrich (1977), Path- and area-integrated rainfall measurement by microwave attenuation in the 1-3 cm band, Journal of Applied Meteorology, 16, 1322-1331, doi: 10.1175/1520-0450(1977) $016<1322$ :PAAIRM $>2.0$.CO $; 2$.

Atlas, D., and C. W. Ulbrich (2006), Drop size spectra and integral remote sensing parameters in the transition from convective to stratiform rain, Geophysical Research Letters, 33(L16803), doi: 10.1029/2006GL026824.

Battan, L. (1973), Radar observation of the atmosphere.

Beard, K. (1976), Terminal velocity and shape of cloud and precipitation drops aloft, Journal of the Atmospheric Sciences, 33, 851-864, doi: 10.1175/1520-0469(1976)033<0851:TVASOC > 2.0.CO;2.

Beard, K. (1977), Terminal velocity adjustment for cloud and precipitation drops aloft, Journal of the Atmospheric Sciences, 34, 1293-1298, doi: 10.1175/1520-0469(1977)034<1293:TVAFCA > 2.0.CO;2.

Berne, A., and R. Uijlenhoet (2007), Path-averaged rainfall estimation using microwave links: Uncertainty due to spatial rainfall variability, Geophysical Research Letters, 34(7), L07,403, doi: 10.1029/2007GL029409.

Berne, A., G. Delrieu, J.-D. Creutin, and C. Obled (2004), Temporal and spatial resolution of rainfall measurements required for urban hydrology, Journal of Hydrology, 299, 166-179, doi: 10.1016/j.hydrol.2004.08. 002.

Brauer, C. C., A. Overeem, H. Leijnse, and R. Uijlenhoet (2016), The effect of differences between rainfall measurement techniques on groundwater and discharge simulations in a lowland catchment, Hydrological Processes, 30, 3885-3900, doi: 10.1002/hyp.10898.

Browning, K., and C. Collier (1989), Nowcasting of precipitation systems, Reviews of Ceophysics, 27, 345-370, doi: 10.1029/RG027i003p00345.

Cao, Q., G. Zhang, E. A. Brandes, and T. J. Schuur (2010), Polarimetric radar rain estimation through retrieval of drop size distribution using a bayesian approach, Journal of Applied Meteorology and Climatology, 49, 973-990, doi: 10.1175/2009JAMC2227.1.

Cherkassky, D., J. Ostrometzky, and H. Messer (2014), Precipitation classification using measurements from commercial microwave links, IEEE Transactions on Ceoscience and Remote Sensing, 52(5), 2350-2356, doi: 10. 1109/TGRS.2013.2259832.

Chwala, C., A. Gmeiner, W. Qiu, S. Hipp, D. Nienaber, U. Siart, T. Eibert, M. Pohl, J. Seltmann, J. Fritz, and H. Kunstmann (2012), Precipitation observation using microwave backhaul links in the alpine and prealpine region of southern germany, Hydrology and Earth System Sciences, 16, 2647-2661, doi: 10.5194/ hess-16-2647-2012.

Chwala, C., H. Kunstmann, S. Hipp, and U. Siart (2013), A monostatic microwave transmission experiment for line integrated precipitation and humidity remote sensing, Atmospheric Research, 144, 57-72, doi: 10.1016/ j.atmosres.2013.05.014.

Chwala, C., F. Kleis, and H. Kunstmann (2016), real-time data acquisition of commercial microwave link networks for hydrometeorological applications, Atmospheric Measurement Techniques, 9, 991-999, doi: 10. 5194/amt-9-991-2016. 
Ciach, G. J., and W. F. Krajewski (2006), Analysis and modeling of spatial correlation structure in small-scale rainfall in central Oklahoma, Advances in Water Resources, 29, 1450-1463, doi: 10.1016/j.advwatres.2005. 11.003.

David, N., P. Alpert, and H. Messer (2013), The potential of commercial microwave networks to monitor dense fog-feasibility study, Journal of Ceophysical Research: Atmospheres, 118(11), 750-761, doi: 10.1002/ 2013JD020346.

De Vos, L. W., H. Leijnse, A. Overeem, and R. Uijlenhoet (2017), The potential of urban rainfall monitoring with crowdsourced automatic weather stations in Amsterdam, Hydrology and Earth System Sciences, 21, 765-777, doi: 10.5194/hess-21-765-2017.

De Vos, L. W., T. H. Raupach, H. Leijnse, A. Overeem, A. Berne, and R. Uijlenhoet (2018), High-resolution simulation study exploring the potential of radars, crowdsourced personal weather stations, and commercial microwave links to monitor small-scale urban rainfall, Water Resources Research, 54, doi: 10.1029/ 2018WR023393.

Doumounia, A., M. Gosset, F. Cazenave, and F. K. Modeste, Zougmore (2014), Rainfall monitoring based on microwave links from cellular telecommunication networks: First results from a West African test bed, Geophysical Research Letters, 41(16), 6016-6022, doi: 10.1002/2014GL060724.

Droste, A., J. Pape, A. Overeem, H. Leijnse, G. Steeneveld, A. V. Delden, and R. Uijlehoet (2017), Crowdsourcing urban air temperatures through smartphone battery temperatures in São Paulo, Brazil, J. Atmos. Oceanic Technol., 34, 1853-1866, doi: 10.1175/JTECG-D-16-0150.1.

Ericsson (2017), Ericsson microwave outlook 2017, Tech. rep.

Ericsson (2018), Ericsson microwave outlook 2018, Tech. rep.

Fabry, F. (2015a), Radar Meteorology: Principles and Practice, Cambridge University Press, doi: 10.1017/ CBO9781107707405.

Fabry, F. (2015b), The added value of dual polarization, in Radar Meteorology: Principles and Practice, pp. 92-114, Cambridge University Press, doi: 10.1017/CBO97811077074505.007.

Corgucci, E., V. Chandrasekar, V. N. Bringi, and G. Scarchilli (2002), Estimation of raindrop size distribution parameters from polarimetric radar measurements, Journal of Atmospheric Sciences, 59, 2373-2384, doi: 10. 1175/1520-0469(2002)059<2373:EORSDP > 2.0.CO;2.

Cosset, M., H. Kunstmann, F. Zougmore, F. Cazenave, H. Leijnse, R. Uijlenhoet, C. Chwala, F. keis, A. Doumounia, B. Boubacar, M. Kacou, P. alpert, H. Messer, J. Rieckermann, and J. Hoedjes (2016), Improving rainfall measurement in gauge poor regions thanks to mobile telecommunication networks, Bull. Amer. Meteor. Soc., 97, ES49-ES51, doi: 10.1175/BAMS-D-15-00164.1.

Habib, E., and W. F. Krajweski (2002), Uncertainty analysis of the TRMM ground-validation radar-rainfall products: application to the TEFLUN-B field campaign, Journal of Applied Meteorology, 41, 558-572, doi: 10.1175/ 1520-0450(2002)041<0558:UAOTTG > 2.0.CO;2.

Hazenberg, P., H. Leijnse, and R. Uijlenhoet (2011), Radar rainfall estimation of stratiform winter precipitation in the Belgian Ardennes, Water Resources Research, 47, W02,507, doi: 10.1029/2010WR009068.

Hazenberg, P., P. Torfs, H. Leijnse, G. Delrieu, and R. Uijlenhoet (2013), Identification and uncertainty estimation of vertical reflectivity profiles using a Lagrangian approach to support quantitative precipitation measurements by weather radar, Journal ofCeophysical Research: Atmospheres, 118, 10,243-10,261, doi: 10.1002/jgrd. 50726 .

Hazenberg, P., H. leijnse, and R. Uijlenhoet (2014), The impact of reflectivity correction and accounting for raindrop size distribution variability to improve precipitation estimation by weather radar for an extreme lowland mesoscale convective system, Journal of Hydrology, 519, 3410-3425, doi: 10.1016/j.hydrol.2014.09. 057.

Holt, A., G. Kuznetsov, and A. Rahimi (2003), Comparison of the use of dual-frequency and single-frequency attenuation for the measurement of path-averaged rainfall along a microwave link, IEEE Proceedings - Microwaves, Antennas and Propagation, 150(5), 315-320, doi: 10.1049/ip-map:20030616.

Islam, T., M. A. Rico-Ramirez, and D. Han (2012), Tree-based genetic programming approach to infer microphysical paramters of the DSDs from the polarization diversity measurements, Computers and Ceosciences, 48, 20-30, doi: 10.1016/j.cageo.2012.05.028.

ITU (2005), ITU-R: Recommendation: P.838-3. specific attenuation model for rain for use in prediction methods, Tech. rep. 
Jaffrain, J., and A. Berne (2012), Quantification of the small-scale spatial structure of the raindrop size distribution from a network of disdrometers, Journal of applied meteorology and climatology, 51, 941-953, doi: 10.1175/JAMC-D-11-0136.1.

Jaffrain, J., A. Studzinski, and A. Berne (2011), A network of disdrometers to quantify the small-scale variability of the raindrop size distribution, Water Resources Research, 47(3), WoOH06, doi: 10.1029/ 2010WR009872.

Jameson, A., M. Larsen, and A. Kostinski (2015), Disdrometer network observations of finescale spatial-temporal clustering in rain, journal of the Atmospheric Sciences, 72, 1648-1666, doi: 10.1175/JAS-D-14-0136.1.

Joss, J., and A. Waldvogel (1967), A raindrop spectrograph with automatic analysis, Pure Appl. Ceophys., 68, 240246.

Kidd, C., A. Becker, C. J. Huffman, C. L. Muller, P. Joe, C. Skofronick-Jackson, and D. B. Kirschbaum (2017), So, how much of the earth's surface is covered by rain gauges?, Bulletin of the American Meteorological Society, 98 , 69-78, doi: 10.1175/BAMS-D-14-00283.1.

Krajewski, W. F., G. J. Ciach, and E. Habib (2003), An analysis of small-scale rainfall variability in different climatic regimes, Hydrological Sciences Journal, 48, 151-162, doi: 10.1623/hysj.48.2.151.44694.

Leijnse, H., R. Uijlenhoet, and ]. Stricker (2007a), Rainfall measurement using radio links from cellular communication networks, Water Resources Research, 43, W03,201, doi: 10.1029/2006WR005631.

Leijnse, H., R. Uijlenhoet, and ]. Stricker (2007b), Hydrometeorological application of a microwave link: 1. evaporation, Water Resources Research, 43(4), W04,416, doi: 10.1029/2006WR005631.

Leijnse, H., R. Uijlenhoet, and ]. Stricker (2007c), Hydrometeorological application of a microwave link: 2. precipitation, Water Resources Research, 43, W04,417, doi: 10.1029/2006WR004989.

Leijnse, H., R. Uijlenhoet, and ]. Stricker (2008), Microwave link rainfall estimation: Effects of link length and frequency, temporal sampling, power resolution, and wet antenna attenuation, Advances in Water Resources, 31, 1481-1493, doi: 10.1016/j.advwatres.2008.03.004.

Leijnse, H., R. Uijlenhoet, and A. Berne (2010), Errors and uncertainties in microwave link rainfall estimation explored using drop size measurements and high-resolution radar data, Journal of Hydrometeorology, 11, 13301344, doi: 10.1175/2010JHM1243.1.

Leinonen, J. (2014), High-level interface to t-matrix scattering calculations: architecture, capabilities and limitations, Optics Express, 22(2), 1655-1660, doi: 10.1364/OE.22.001655.

Liebe, H. J., T. Manabe, and G. A. Hufford (1989), Millimeter-wave attenuation and delay rates due to fog/cloud conditions, IEEE Transactions on Antennas and Propagation, 37(12), 1617-1612, doi: 10.1109/8.45106.

Liebe, H. J., G. A. Hufford, and T. Manabe (1991), A model for the complex permittivity of water at frequencies below1 THz, International Journal of Infrared and Millimeter Waves, 12(7), 659-675, doi: 10.1007/BF01008897.

Löffler-Mang, M., and J. Joss (2000), An optical disdrometer for measuring size and velocity of hydrometeors, Journal of Atmospheric and Oceanic Technology, 17(2), 130-139, doi: 10.1175/1520-0426(2000)017<0130: AODFMS $>2.0 ; 2$.

Marshall, J., and W. M. Palmer (1948), The distribution of raindrops with size, Journal of Meteorology, 5, 165-166, doi: 10.1175/1520-0469(1948)005<0165:TDORWS > 2.0.CO;2.

Matrosov, S. Y. (2007), Modeling backscatter properties of snowfall at millimeter wavelengths, Journal of the Atmospheric Sciences, 64, 1727-1736, doi: 10.1175/JAS3904.1.

McCabe, M. F., M. Rodell, D. E. Alsdorf, D. G. Miralles, R. Uijlenhoet, W. Wagner, A. Lucieer, R. Houborg, N. E. Verhoest, T. E. Franz, J. Shi, H. Gao, and E. F. Wood (2017), The future of earth observation in hydrology, Hydrology and Earth System Sciences, 21, 3879-3914, doi: 10.5194/hess-21-3879-2017.

Messer, H., A. Zinevich, and P. Alpert (2006), Environmental monitoring by wireless communication networks, Science, 312, 713, doi: 10.1126/science.1120034.

Mie, G. (1908), Beiträge zur optiek trüber medien, speziell koloidaler mettalösungen, Annalen der Physik(Berlin), 330, 377-445, doi: 10.1002/andp.19083300302.

Minda, H., and K. Nakamura (2005), High temporal resolution path-average rain gauge with $50-\mathrm{CHz}$ band microwave, Journal of Atmospheric and Oceanic Technology, 22, 165-179, doi: 10.1175/JTECH-1683.1.

Mishchenko, M. I. (2000), Calculation of the amplitude matrix for a nonspherical particle in a fixed orientation, Applied Optics, 39(6), 1026-1031, doi: 10.1364/AO.39.001026. 
Mishchenko, M. I., and L. D. Travis (1998), Capabilities and limitations of a current FORTRAN implementation of the T-matrix method for randomly oriented, rotationally symmetric scatterers, Journal of Quantitative Spectroscopy and Radiative Transfer, 60(3), 309-324, doi: 10.1016/S0022-4073(98)00008-9.

Mishchenko, M. I., L. D. Travis, and D. W. Mackowski (1996), T-matrix computations of light scattering by nonspherical particles: a review, Journal of Quantitative Spectroscopy and Radiative Transfer, 55(5), 535-575, doi: 10.1016/0022-4073(96)00002-7.

Olsen, R., D. V. Rogers, and D. Hodge (1978), The $a r^{b}$ relation in the calculation of rain attenuation, IEEE Transactions on Antenna Propagation, AP-26, 318-329, doi: 10.1109/TAP.1978.1141845.

Orlanski, I. (1975), A rational subdivision of scales for atmospheric sciences, Bulletin of the American Meteorological society, 56, 527-530.

Ostrometzky, ]., D. Cherkassky, and H. Messer (2015), Accumulated mixed precipitation estimation using measurements from multiple microwave links, Advances in Meteorology, 2015, Article ID 707,646, doi: 10.1155/ $2015 / 707646$.

Overeem, A., T. A. Buishand, and I. Holleman (2009a), Extreme rainfall analysis and estimation of depth-duration-frequency curves using weather radar, Water. Resour. Res., 45, n/a-n/a, doi: 10.1029/ 2009WR007869, w10424.

Overeem, A., I. Holleman, and A. Buishand (2009b), Derivation of a 10-year radar-based climatology of rainfall, J. Appl. Meteorol. Clim., 48, 1448-1463, doi: 10.1175/2009JAMC1954.1.

Overeem, A., H. Leijnse, and R. Uijlenhoet (2011), Measuring urban rainfall using microwave links from commercial cellular communication networks, Water Resources Research, 47, doi: 10.1029/2010WR010350, w12505.

Overeem, A., H. Leijnse, and R. Uijlenhoet (2013), Country-wide rainfall maps from cellular communication networks, PNAS, 110(8), 2741-2745, doi: 10.1073/pnas.1217961110.

Overeem, A., H. Leijnse, and R. Uijlenhoet (2016a), Retrieval algorithm for rainfall mapping from microwave link in a cellular communication network. Atmospheric Measurement Techniques, 9, 2425-2444, doi: 10. 5194/amt-9-2425-2016.

Overeem, A., H. Leijnse, and R. Uijlenhoet (2016b), Two and a half years of country-wide rainfall maps using radio links from commercial cellular telecommunication networks, Water Resources Research, 52(10), 80398065, doi: 10.1002/2016WR019412.

Paulson, K., and A. Al-Mreri (2011), A rain height model to predict fading due to wet snow on terrestrial links, Radio Science, 46(4), RS4010, doi: 10.1029/2010RS004555.

Peleg, N., M. Ben-Asher, and E. Morin (2013), Radar subpixel-scale rainfall variability and uncertainty: lessons learned from observations of a dense rain-gauge network, Hydrology and Earth System Sciences, 17, 21952208, doi: 10.5194/hess-17-2195-2013.

Rahimi, A., A. Holt, G. Upton, and R. Cummings (2003), Use of dual-frequency microwave links for measuring path-averaged rainfall, Journal of Ceophysical Research: Atmospheres, 108(D15), 2156-2202, doi: 10.1029/ 2002JD003202.

Raupach, T. H., and A. Berne (2015), Correction of raindrop size distributions measured by Parsivel disdrometers, using a two-dimensional video disdrometer as a reference, Atmospheric Measurement Techniques, 8, 343365, doi: 10.5194/amt-8-343-2015.

Raupach, T. H., and A. Berne (2016), Spatial interpolation of experimental raindrop size distribution spectra, Quarterly Journal of the Royal Meteorological Society, 142, 125-137.

Raupach, T. H., and A. Berne (2017), Retrieval of the raindrop size distribution from polarimetric radar data using double-moment normalization, Atmospheric Measurement Techniques, 10, 2573-2594, doi: 10.5194/ amt-10-2573-2017.

Rincon, R. F., and R. H. Lang (2002), Microwave link dual-wavelength measurements of path-average attenuation for the estimation of drop size distributions and rainfall, IEEE Transactions on Ceoscience and Remote Sensing, 40(4), 760-770, doi: 10.1109/TGRS.2002.1006324.

Rios Gaona, M. F., A. Overeem, H. Leijnse, and R. Uijlenhoet (2015), Measurement and interpolation uncertainties in rainfall maps from cellular communication networks, Hydrology and Earth System Sciences, 19, 35713584, doi: 10.5194/hess-19-3571-2015.

Rios Gaona, M. F., A. Overeem, H. Leijnse, and R. Uijlenhoet (2016), First-year evaluation of GPM rainfall over 
the netherlands: IMERG day 1 final run (V03D), Journal of Hydrometerology, 17, 2799-2814, doi: 10.1175/ JHM-D-16-0087.1.

Rios Gaona, M. F., A. Overeem, A. Brasjen, J. F. Meirink, H. Leijnse, and R. Uijlenhoet (2017), Evaluation of rainfall products derived from satellites and microwave links for the netherlands, IEE Transactions on Ceoscience and Remote Sensing, 55, 6849-6859, doi: 10.1109/TGRS.2017.2735439.

Rios Gaona, M. F., A. Overeem, T. H. Raupach, H. Leijnse, and R. Uijlenhoet (2018), Rainfall retrieval with commercial microwave links in São Paulo, Brazil, Atmospheric Measurement Techniques, 11, 4465-4476, doi: 10.5194/amt-11-4465-2018.

Salles, C., and J. Poesen (1999), Performance of an optical spesctro pluviometer in measuring basic rain erosivity characteristics, Journal of Hydrology, 218, 142-156, doi: 10.1016/S0022-1694(99)00031-1.

Salles, C., and J. Poesen (2000), Rain properties controlling soil splash detachment, Hydrological Processes, 14, 271-282, doi: 10.1002/(SICI)1099-1085(20000215)14:2<271::AID-HYP925>3.0.CO;2-J.

Salles, C., J. Poesen, and L. Borselli (1999), Measurement of simulated drop size distribution with an optical spectro pluviometer: Sample size considerations, Earth Surface Processes and Landforms, 24, 545-556, doi: 10. 1002/(SICI)1096-9837(199906)24:6<545::AID-ESP3>3.0.CO;2-D.

Schilling, W. (1991), Rainfall data for urban hydrology: what do we need?, Atmospheric Research, 27(1-3), 5-21, doi: 10.1016/0169-8095(91)90003-F.

Schleiss, M., and A. Berne (2010), Identification of dry and rainy periods using telecommunication microwave links, IEEE Geoscience and Remote Sensing Letters, 7(3), 611-615, doi: 10.1109/LGRS.2010.2043052.

Schleiss, M., J. Rieckermann, and A. Berne (2013), Quantification and modelling of wet-antenna attenuation for commercial microwave links, IEEE Ceoscience Remote Sensing Letters, 10, 1195-1199, doi: 10.1109/LGRS. 2012.2236074 .

Schuurmans, J., M. Bierkens, and E. Pebesma (2007), Automatic prediction of high-resolution daily rainfall fields for multiple extents: The potential of operational radar, Journal of Hydrometeorology, 8, 1204-1224, doi: 10.1175/2007JHM792.1.

Schönhuber, M., G. Lammer, and W. L. Randeu (2008), The 2d-video-distrometer, in Precipitation: Advances in measurement, estimation and prediction, pp. 3-31, Springer.

Skofronick-Jackson, C., W. Berg, C. Kidd, D. B. Kirschbaum, W. A. Peterson, , G. J. Huffman, and Y. N. Takayabu (2018), Global precipitation measurement (GPM): Unified precipitation estimation from space, in Remote Sensing of Clouds and Precipitation, edited by C. Andronache, pp. 175-193, Springer, Cham.

Strangeways, I. (2010), A history of rain gauges, Weather, 65, 133-138, doi: 10.1002/wea.548.

Tapiador, F., R. Checa, and M. de Castro (2010), An experiment to measure the spatial variability of rain drop size distribution using sixteen laser disdrometers, Geophysical Research Letters, 37, doi: 10.1029/ 2010GL044120.

Thurai, M., G. Huang, V. Bringi, W. Randeu, and M. Schönhuber (2007), Drop shapes, model comparisons, and calculations of polarimetric radar parameters in rain, Journal of Atmospheric and Oceanic Technology, 24, 10191032

Tokay, A., and D. A. Short (1996), Evidence from tropical raindrop spectra of the origin of rain from stratiform versus convective clouds, Journal of Applied Meteorology, 35, 355-371, doi: 10.1175/1520-0450(1996) $035<0355$ :EFTRSO $>2.0 . \mathrm{CO} ; 2$.

Tokay, A., and K. Öztürk (2012), An experimental study of the small-scale variability of rainfall, Journal of Hydrometeorology, 13, 352-365, doi: 10.1175/JHM-D-11-014.1.

Uijlenhoet, R., and J. Stricker (1999), A consistent rainfall parameterization based on the exponential raindrop size distribution, Journal of Hydrology, 218, 101-127, doi: 10.1016/S0022-1694(99)00032-3.

Uijlenhoet, R., J. A. Smith, and M. Steiner (2003), The microphysical structure of extreme precipitation as inferred from ground-based raindrop spectra, Journal of the Atmospheric Sciences, 60, 1220-1238, doi: 10.1175/ 1520-0469(2003)60<1220:TMSOEP $>2.0$.CO 2 .

Uijlenhoet, R., J. M. Porrà, D. Sempere Torres, and].-D. Creutin (2006), Analytical solutions to sampling effects in drop size distribution measurements during stationary rainfall: Estimation of bulk rainfall variables, Journal of Hydrology, 328, 65-82, doi: 10.1016/j.jhydrol.2005.11.043.

Uijlenhoet, R., J.-M. Cohard, and Cosset (2011), Path-average rainfall estimation from optical extinction measurements using a large-aperture scintillometer, Journal of Hydrometeorology, 12, 955-972. 
Uijlenhoet, R., A. Overeem, and H. Leijnse (2018), Opportunistic remote sensing of rainfall using microwave links from cellular communication networks, Wiley Interdisciplinary Reviews: water, 5, e1289, doi: 10.1002/ wat2.1289.

Ulbrich, C. W. (1983), Natural variations in the analytical form of the raindrop size distribution, Journal of Climate and Applied Meteorology, 22, 1764-1775, doi: 10.1175/1520-0450(1983)022<1764:NVITAF >2. $0 . \mathrm{CO} ; 2$.

Upton, G., A. Holt, R. Cummings, A. Rahimi, and ]. Goddard (2005), Microwave links: The future for urban rainfall measurement, Atmospheric Research, 77, 300-312, doi: 10.1016/j.atmosres.2004.10.009.

Van de Beek, C., H. Leijnse, P. Torfs, and R. Uijlenhoet (2011), Seasonal semi-variance of Dutch rainfall at hourly to daily scales, Advances in Water Resources, 15, 171-183, doi: 10.1016/j/advwatres.2012.03.023.

Van der Hulst, H. (1957), Light scattering by small particles, John Wiley \& Sons.

Van Leth, T. C., A. Overeem, H. Leijnse, and R. Uijlenhoet (2018), A measurement campaign to assess sources of error in microwave link rainfall estimation, Atmospheric Measurement Techniques, 11, 4645-4669.

Vargaftik, N., B. Volkov, and L. Voljak (1983), International tables of the surface tension of water, Journal of Physical an Chemical Reference Data, 12(3), 817-820, doi: 10.1063/1.555688.

Villarini, G., P. V. Mandapaka, W. F. Krajewski, and R. J. Moore (2008), Rainfall and sampling uncertainties: A rain gauge perspective, Journal ofCeophysical Research, 113, D11,102, doi: 10.1029/2007JD009214.

Vulpiani, G., F. S. Marzano, V. Chandrasekar, A. Berne, and R. Uijlenhoet (2006), Polarimetric weather radar retrieval of raindrop size distribution by means of a regularized artificial neural network, IEEE Transactions on Ceoscience and Remote Sensing, 44(11), 3262-3275, doi: 10.1109/TGRS.2006.878438.

Wang, Z., M. Schleiss, J. Jaffrain, A. Berne, and J. Rieckermann (2012), Using markov switching models to infer dry and rainy periods from telecommunication microwave link signals, Atmospheric Measurement Techniques, 5, 1847-1859, doi: 10.5194/amt-5-1847-2012.

Waterman, P. (1965), Matrix formulation of electromagnetic scattering, Proceedings of the IEEE, 53(8), 805-812, doi: 10.1109/PROC.1965.4058

Wessels, H. (1972), Metingen van regendruppels te De Bilt, Tech. rep., KNMI.

Zhang, G., J. Vivekanandan, and E. Brandes (2001), A method for estimating rain rate and drop size distribution from polarimetric radar measurements, IEEE Transactions on Ceoscience and Remote Sensing, 39(4), 830-841, doi: $10.1109 / 36.917906$.

Zhang, G., J. Vivekanandan, E. A. Brandes, R. Meneghini, and T. Kozu (2003), The shape-slope relation in observed gamma raindrop size distributions: statistical error of useful information?, 20, 1106-1119, doi: 10.1175/1520-0426(2003)020<1106:TSRIOG > 2.0.CO;2.

Zinevich, A., P. Alpert, and H. Messer (2008), Estimation of rainfall fields using commercial microwave communication networks of variable density, Advances in Water Resources, 31, 1470-1480, doi: 10.1016.j. advwatres.2008.03.003.

Zinevich, A., H. Messer, and P. Alpert (2009), Frontal rainfall observation by a commercial microwave communication network, Journal of Applied Meteorology and Climatology, 48, 1317-1334, doi: 10.1175/ 2008JAMC2014.1.

Zinevich, A., H. Messer, and P. Alpert (2010), Prediction of rainfall intensity measurement errors using commercial microwave communication links, Atmospheric Measurement Techniques, 3, 1385-1402, doi: 10.5194/ amt-3-1385-2010. 


\section{Statement of authorship contribution}

Chapter 1 Introduction. The general subject of the research was suggested by my (co-)promotors. I worked out the research plan and specific direction of the research in detail with approval of my (co-)promotors. I did the literature research and wrote the text with minor corrections from my (co-)promotors.

Chapter 2 A campaign to assess error sources in microwave link rainfall estimation. The initial experiment was set up and conceptualized by my (co-)promotors together with a technician and a previous PhD candidate, to which I made significant additions. Data was collected mainly by me, with some help of Aart Overeem and a technician. I built an extensive software library to process the data and used this to analyze and interpret the data, which have been made available through the 4TU Centre for Research data. I wrote the first draft for this chapter and after critical revision by my (co-)promotors and anonymous referees wrote the final version.

Chapter 3 Rainfall spatio-temporal correlation and intermittency structure. The idea for this chapter came from a discussion between me and Remko Uijlenhoet. Data that I had already collected in the previous experiment was used together with additional data that Aart Overeem helped me obtain. I performed the analysis and interpreted the results. I wrote the first draft and after critical revision by my (co-)promotors wrote the final version.

Chapter 4 Estimating raindrop size distributions using microwave link measurements. The conception of this chapter was solely my idea, with some suggestions from Remko Uijlenhoet. I devised the algorithm, wrote the necessary software to perform the retrievals and analyzed the results, with feedback by Hidde Leijnse and Remko Uijlenhoet. I wrote the first draft and after critical revision by my (co-)promotors wrote the final version.

(Chapter 5) Synthesis. The ideas and arguments put forward in this chapter are my own, although it is unavoidable that these were influenced to some extent by the ideas of my (co-)promotors. I wrote the text with only minor corrections by my (co-)promotors. 


\section{Dankwoord}

Het schrijven van een proefschrift is iets wat je maar eens in je leven doet. Het is een zogenaamde proeve van bekwaamheid en een persoonlijk statement. Het eindpunt van een eenzame reis en het resultaat van jouw noeste arbeid. Jouw Levenswerk.

Dat is althans de idee. In werkelijkheid is een proefschrift niet het eind van de reis, maar eerder een overstap op Utrecht Centraal. Belangrijker nog: Het is het werk van velen. Dit is dan ook bij uitstek de plek om dit misverstand recht te zetten.

In de eerste plaats is daar natuurlijk de promotor. Hoewel het inderdaad klopt dat er maar weinig mensen zijn die meer dan één proefschrift op hun naam hebben staan, heeft de promotor toch veel promotietrajecten meegemaakt en is zijn ervaring onontbeerlijk. Ik heb veel geluk gehad met Remko Uijlenhoet als promotor, want ondanks de vele promovendi die er in de loop der jaren bij hem langs zijn gekomen blijft bij hem elke promotie bijzonder. Ik wil hem dan ook bedanken voor de grote aandacht en betrokkenheid die hij altijd voor mij en mijn werk heeft getoond. Voor het feit dat ik altijd bij hem binnen kon lopen en de spontane discussies en vergezichten die daar konden onstaan. Zijn aanstekelijke enthousiasme en zijn onverslaanbaar goede humeur maken het altijd prettig om met hem samen te werken.

Daarnaast zijn daar natuurlijk mijn co-promotoren Aart Overeem en Hidde leijnse die me altijd van waardevol commentaar en advies konden voorzien. Daarnaast wil ik Aart nog in het bijzonder bedanken voor het bijbrengen van de kunst van de flauwe woordgrap. Mijn werk is daarnaast naturlijk ook schatplichtig aan het eerdere promotieonderzoek van Hidde. I $\mathrm{k}$ wil verder Hidde nog bedanken voor de extra aandacht in de laatste weken.

Er is bij mij geen twijfel dat de naam die tot nog toe het meest onderbelicht is gebleven die van Pieter Hazenberg is. Zonder hem zou het experimentele werk zeer lastig, zo niet onmogelijk, geweest zijn. Ik wil hem daarom bedanken voor alle arbeid en expertise die hij voor dit project heeft ingezet en ook voor alle gezamelijke onderhoudstripjes naar de daken van Biotechnion, Forum en andere locaties. Ik vond dit ook altijd zeer gezellig en leerzaam.

Vele anderen hebben op een of andere manier bijdrages geleverd aand dit werk: Ralph Koppelaar en Ronald Kloeg van T-mobile, bedankt voor het regelen van de links, het beschikbaar maken van data en de welgemeende interesse tijdens de gebruikerscommissie vergaderingen. Alexis Berne voor het uitlenen van de parsivels, die uiteindelijk het experiment een stuk interessanter hebben kunnen maken. De gebouwbeheerders bij De Bongerd, NVWA en Biotechnion die Pieter en mij in staat hebben gesteld die instrumenten bij hen op het dak te installeren. Tim Raupach voor het ter beschikking stellen van zijn simu- 
latiedata en verder iedereen die ik hier vergeten ben.

De reis is ook niet zo eenzaam als die lijkt, want er zijn er meer die dezelfde weg bewandelen. Ik wil dan ook mijn medereizigers in coupé D105 (aka "de afgang" aka "het verdomhoekje") Timo, Femke, Tjitske en Joost bedanken voor de ontspannen en open sfeer. Er was gelukkig altijd tijd voor meligheid, maar ik ben vooral blij dat hier ook alle serieuzere zaken altijd zeer openhartig besproken konden worden. Daarnaast wil ik ook Lotte bedanken omdat ze me meer dan eens uit de brand heeft geholpen. (Tevens excuses aan de mensen van de CeoDesk die ik steevast op het laatste moment lastig val). Ook alle andere HWM collega's wil ik bedanken voor de gezelligheid in de groep. Ik ben blij om in dit schuitje mee te hebben gevaren (en allicht nog een tijdje te blijven doen).

Tot slot zou je in de academische bubbel nogal eens vergeten dat de gelegenheid tot promoveren geen vanzelfsprekendheid is maar een voorrecht. Niet elk dorp heeft een bushalte en soms moet je eerst een heel eind lopen. I $\mathrm{k}$ wil daarom de mensen bedanken die mij in eerdere ettappes hebben gesteund en die het voor mij mogelijk hebben gemaakt om aan deze reis te beginnen: Jacqueline, Marlies, Anja, Arend, Ceert-Jan en alle anderen. Uiteraard mag in dit rijtje mijn familie niet ontbreken: Jesse, Tristan, Inge en vooral pap en mam die er altijd in hebben geloofd. (Ook bedankt voor de kaft mam; Over druppelvorm moeten we het nog eens hebben). 


\section{SENSE}

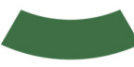

Netherlands Research School for the

Socio-Economic and Natural Sciences of the Environment

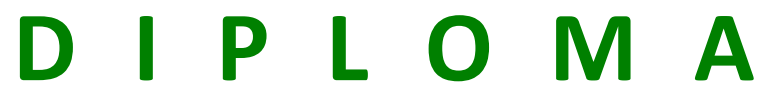

For specialised PhD training

The Netherlands Research School for the

Socio-Economic and Natural Sciences of the Environment

(SENSE) declares that

\section{Thomas Christiaan van Leth}

born on 5 June 1987, Rotterdam, The Netherlands

has successfully fulfilled all requirements of the

Educational Programme of SENSE.

Wageningen, 26 August 2019

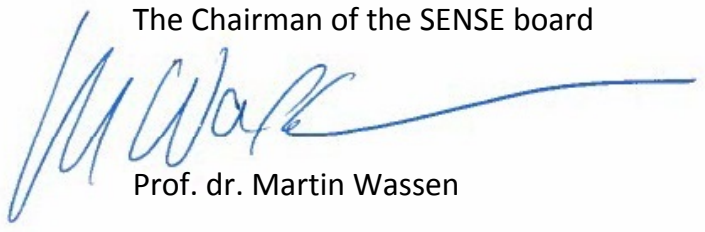

the SENSE Director of Education

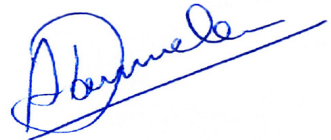

Dr. Ad van Dommelen

The SENSE Research School has been accredited by the Royal Netherlands Academy of Arts and Sciences (KNAW)

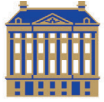

$\begin{array}{lllllllllllllllllllllll}K & O & N & I & N & K & L & I & J & K & E & N & E & D & E & R & L & A & N & D & S & E\end{array}$

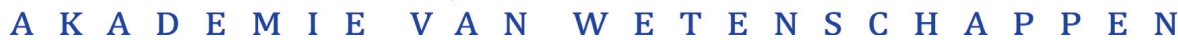




\section{SENSE}

The SENSE Research School declares that Thomas Christiaan van Leth has successfully fulfilled all requirements of the Educational PhD Programme of SENSE with a work load of $34.0 \mathrm{EC}$, including the following activities:

\section{SENSE PhD Courses}

- Environmental research in context (2015)

- Research in context activity: 'Advising cellphone operators in Sri Lanka, Nigeria and Bangladesh on the feasibility of using their networks for estimating rainfall and the value of providing services based on this rainfall estimation to their customers' (2019)

\section{Other PhD and Advanced MSc Courses}

- Scientific publishing, Wageningen Graduate Schools (2018)

- Reviewing a scientific paper, Wageningen Graduate Schools (2018)

- Start to teach, Wageningen Graduate Schools (2018)

- Quantitative precipitation estimation, UC Irvine (2019)

- Extreme value analysis, UC Irvine (2019)

\section{Didactic Skills Training}

o Supervising five BSc students with thesis (2015-2017)

- Teaching in the MSc course 'Environmental data collection and analysis' (2016-2018)

o Teaching in the MSc course 'Urban hydrometeorology' (2016-2018)

\section{Management Skills Training}

- Member WIMEK PhD Council (2016-2017)

- Member Wageningen PhD council (2017)

\section{Oral Presentations}

- Wageningen Urban Rainfall Experiment 2014. EGU general assembly, 13-17 April 2015, Vienna, Austria

- Wageningen Urban Rainfall experiment 2014-2015: Preliminary results. EGU general assembly, 18-22 April 2016, Vienna, Austria

- Opportunistic sensing of rainfall using microwave links from cellular communication networks in Europe, Africa and Asia. International Precipitation Conference (IPC12), 1821 June 2019, Irvine, United States of America

SENSE Coordinator PhD Education

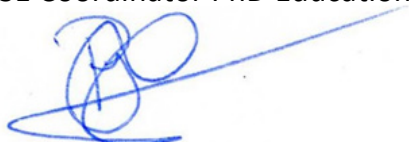

Dr. ir. Peter Vermeulen 
The research described in this thesis was financially supported by the Netherlands Organization for Scientific Research NWO-TTW (project 11944). Financial support from Wageningen University for printing this thesis is gratefully acknowledged.

Cover design by Veronica van Leth 


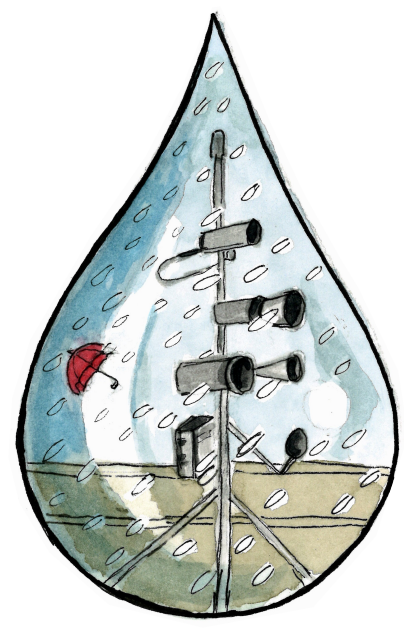

